TRANSACTIONS OF THE

AMERICAN MATHEMATICAL SOCIETY

Volume 362, Number 10, October 2010, Pages 5125-5168

S 0002-9947(10)04950-0

Article electronically published on May 10, 2010

\title{
VARIATIONAL PRINCIPLE FOR SPREADING SPEEDS AND GENERALIZED PROPAGATING SPEEDS IN TIME ALMOST PERIODIC AND SPACE PERIODIC KPP MODELS
}

\author{
WENXIAN SHEN
}

\begin{abstract}
Spatial spread and front propagation dynamics is one of the most important dynamical issues in KPP models. Such dynamics of KPP models in time independent or periodic media has been widely studied. Recently, the author of the current paper with Huang established some theoretical foundation for the study of spatial spread and front propagation dynamics of KPP models in time almost periodic and space periodic media. A notion of spreading speed intervals for such models was introduced in the above-mentioned paper and was shown to be the natural extension of the classical concept of the spreading speeds for time independent or periodic KPP models and that it could be used for more general time dependent KPP models. A notion of generalized propagating speed intervals of front solutions and a notion of traveling wave solutions to time almost periodic and space periodic KPP models were also introduced, which are the generalizations of wave speeds and traveling wave solutions in time independent or periodic KPP models.

The aim of the current paper is to gain some further qualitative and quantitative understanding of the spatial spread and front propagation dynamics of KPP models in time almost periodic and space periodic media. By applying the principal Lyapunov exponent and the principal Floquet bundle theory for time almost periodic parabolic equations, we provide various useful estimates for spreading and generalized propagating speeds for such KPP models. Under the so-called linear determinacy condition, we show that the spreading speed interval in any given direction is a singleton (called the spreading speed). Moreover, in such a case we establish a variational principle for the spreading speed and prove that there is a front solution of speed $c$ in a given direction if and only if $c$ is greater than or equal to the spreading speed in that direction. Both the estimates and variational principle provide important and efficient tools for the spreading speeds analysis as well as the spreading speeds computation. Based on the variational principle, the influence of time and space variation of the media on the spreading speeds is also discussed in this paper. It is shown that the time and space variation cannot slow down the spatial spread and that it indeed speeds up the spatial spread except in certain degenerate cases, which provides deep insights into the understanding of the influence of the inhomogeneity of the underline media on the spatial spread in KPP models.
\end{abstract}

Received by the editors May 12, 2008.

2010 Mathematics Subject Classification. Primary 35K55, 35K57; Secondary 35B15, 92D25.

Key words and phrases. Almost periodic KPP model, spreading speed interval, generalized propagating speed interval, traveling wave solution, front solution, principal Lyapunov exponent, principal Floquet bundle, variational principle.

This work was partially supported by NSF grant DMS-0504166. 


\section{INTRODUCTION}

Reaction-diffusion equations arising from many biological, chemical, and engineering problems have two or more different states. Among the most important dynamical issues in such reaction-diffusion equations is the spatial spread and front propagation dynamics such as how fast one of the states spreads, how one state transfers to another state, etc., as time evolves. The current paper deals with the spatial spread and front propagation dynamics for reaction-diffusion equations of the form

$$
\frac{\partial u}{\partial t}=\Delta u+\sum_{i=1}^{N} a_{i}(t, x) \frac{\partial u}{\partial x_{i}}+f(t, x, u), \quad x \in \mathbb{R}^{N} .
$$

We assume that

(H1) $f(t, x, u)$ is $C^{2}$ in $u ; a_{i}(t, x)(i=1,2, \cdots, N), f(t, x, u), \frac{\partial f}{\partial u}(t, x, u)$, and $\frac{\partial^{2} f}{\partial u^{2}}(t, x, u)$ are uniformly almost periodic in $t$ (see Definition A.2 in Appendix A), periodic in $x_{j}$ with period $p_{j}>0(j=1,2, \cdots, N)$, and are globally Hölder continuous in $t, x$.

(H2) (1.1) possesses two states $u^{ \pm}(t, x)$ satisfying the fact that $u^{ \pm}(t, x)$ are periodic in $x_{j}$ with period $p_{j}(j=1,2, \cdots, N)$ and almost periodic in $t$ with $\mathcal{M}\left(u^{ \pm}\right) \subset \mathcal{M}\left(\left\{a_{i}\right\}_{i=1}^{N}, f\right)$ (see Definition A.2 in Appendix A for the definition of $\mathcal{M}(\cdot)), u^{-}(t, x)<u^{+}(t, x)$, and $u=u^{-}(t, x)$ is a linearly unstable solution and $u=u^{+}(t, x)$ is a globally stable solution of (1.1) (with respect to space periodic perturbations) (see Definition [3.1). Without loss of generality, we assume that $u^{-}(t, x) \equiv 0$ and hence $f(t, x, 0)=0$ (for otherwise, we can consider $v(t, x)=$ $\left.u(t, x)-u^{-}(t, x)\right)$.

A typical example of reaction-diffusion equations of the form (1.1) satisfying (H1) and (H2) is the following equation:

$$
\frac{\partial u}{\partial t}=\frac{\partial^{2} u}{\partial x^{2}}+u(1-u), \quad x \in \mathbb{R},
$$

which was introduced in the pioneering papers of Fisher [14 and Kolmogorov, Petrowsky, Piscunov 31 for the evolutionary take-over of a habitat by a fitter genotype, where $u$ is the frequency of one of two forms of a gene. (1.2) possesses two constant states $u^{-}=0, u^{+}=1$, and $u^{-}$is linearly unstable and $u^{+}$is globally stable (with respect to constant perturbations). Fisher in 14 found traveling wave solutions $u(t, x)=\phi(x-c t$ ) connecting 1 and 0 (i.e. $\phi(-\infty)=1, \phi(\infty)=0$ ) of all speeds $c \geq 2$ and showed that there are no such traveling wave solutions of slower speed. He conjectured that the take-over occurs at the asymptotic speed 2. This conjecture was proved in 31] by Kolmogorov, Petrowsky, and Piscunov. That is, they proved that for any nonnegative solution $u(t, x)$ of (1.2), if at time $t=0, u$ is 1 near $-\infty$ and 0 near $\infty$, then $\lim _{t \rightarrow \infty} u(t, c t)$ is 0 if $c>2$ and 1 if $c<2$. In literature, the minimal wave speed $c=2$ is called the spreading speed for (1.2). It is of the following spatially spreading property: for any $\sigma>0$, there is $r_{\sigma}>0 \mathrm{such}$ that for any nonnegative solution $u(t, x)$ of (1.2), if at time $t=0, u(0, x) \geq \sigma$ for $|x| \leq r_{\sigma}$ and $u(0, x)=0$ for $|x| \gg 1$, then

$$
\sup _{|x| \leq c^{\prime} t} u(t, x) \rightarrow 1, \quad \sup _{|x| \geq c^{\prime \prime} t} u(t, x) \rightarrow 0 \quad \text { as } \quad t \rightarrow \infty
$$

for any $c^{\prime}<2$ and $c^{\prime \prime}>2$. 
Observe that (H1) indicates that the environment or medium of the underline system described by (1.1) changes periodically in the space variable and almost periodically in the time variable. The smoothness condition in (H1) is mainly for solutions of related equations to be classical and for $f(t, x, u) \geq\left(\frac{\partial f}{\partial u}(t, x, 0)-\delta\right) u$ to hold for any $\delta>0$ and $t \in \mathbb{R}, x \in \mathbb{R}^{N}, 0<u \ll 1$. (H2) is referred to as a Fisher or KPP type condition and is the condition which generates certain spatial spread and front propagation dynamics in (1.1). In nature, the environments or media of biological models are subject to various seasonal variations. It is then important to investigate which kind of spatial spread and front propagation dynamics there is in time periodic as well as more general time dependent such as time almost periodic variants of the classical Fisher or KPP equation (1.2).

A vast amount of research has been carried out regarding the spatial spread and front propagation dynamics for various variants of (1.2). See, for example, [2, 3], [4], 11], [12, [18, 30], [51, 53], 63], and the references therein for more general time and space independent reaction diffusion equations of KPP type; see [5], 6], 7], 15], 22], 35], 38, 44], 45], 48], 52, 75], and the references therein for space and/or time periodic reaction diffusion equations of KPP type; see [10, [15, [19, [39], [43], [46], 47], 71], 72], and the references therein for KPP models in random media; see [34, [36], 65], 66], and the references therein for time discrete or time periodic KPP models; see [8], 9, [17, 23], 68], 74], and the references therein for spatially discrete KPP models; and see [34, [37, 62, 69, and the references therein for KPP models with delays.

However, there has not yet been much study on the spatial spread and front propagation dynamics for general time almost periodic KPP models. The author of the current paper with Huang developed in [21] some theoretical foundation for the investigation of the spatial spread and front propagation dynamics for general time almost periodic KPP models. This paper is to further investigate the spatial spread and front propagation dynamics for such KPP models.

Recall that when (1.1) is time periodic in $t$ with period $T$, it has been proved that for any $\xi \in \mathbb{R}^{N}$ with $\|\xi\|=1$, there is a $c^{*}(\xi) \in \mathbb{R}$ such that for any $c \geq c^{*}(\xi)$, there is a traveling wave solution connecting $u^{+}$and $u^{-}$and propagating in the direction of $\xi$ with speed $c$, and there is no such traveling wave solution of slower speed. The minimal wave speed $c^{*}(\xi)$ is of some important spreading properties and is called the spreading speed in the direction of $\xi$ (see [5, 6], 7], 34, 44, 45, 66, and the references therein). It has also been proved that if $f(t, x, u) \leq \frac{\partial f}{\partial u}(t, x, 0) u$ for $t \in \mathbb{R}, x \in \mathbb{R}^{N}$, and $u \geq 0$ (which is called the linear determinacy condition in literature), then

$$
c^{*}(\xi)=\inf _{\mu>0} \frac{\lambda^{*}\left(\mu, \xi ; a_{0}\right)}{\mu},
$$

where $\lambda^{*}\left(\mu, \xi ; a_{0}\right)$ is the principal eigenvalue (i.e. the eigenvalue with largest real part) of the following periodic parabolic eigenvalue problem,

$$
\left\{\begin{array}{l}
-\frac{\partial u}{\partial t}+\Delta u+\sum_{i=1}^{N} a_{i}^{\mu, \xi}(t, x) \frac{\partial u}{\partial x_{i}}+a_{0}^{\mu, \xi}(t, x) u=\lambda u, \quad x \in \mathbb{R}^{N}, \\
u\left(t, x_{1}, \cdots, x_{j-1}, x_{j}+p_{j}, x_{j+1}, \cdots, x_{N}\right) \\
\quad=u\left(t, x_{1}, \cdots, x_{j-1}, x_{j}, x_{j+1}, \cdots, x_{N}\right), \quad j=1,2, \cdots, N \\
u(t+T, x)=u(t, x),
\end{array}\right.
$$


$a_{i}^{\mu, \xi}(t, x)=a_{i}(t, x)-2 \mu \xi_{i}(i=1,2, \cdots, N), a_{0}^{\mu, \xi}(t, x)=a_{0}(t, x)-\mu \sum_{i=1}^{N} a_{i}(t, x) \xi_{i}+$ $\mu^{2}$, and $a_{0}(t, x)=f_{u}(t, x, 0):=\frac{\partial f}{\partial u}(t, x, 0)$. In literature, the representation (1.3) for $c^{*}(\xi)$ is called the variational principle for $c^{*}(\xi)$ (see [7, 44, [45, 66, and the references therein for the establishment of the variational principle for certain time independent or periodic KPP models).

As it is known, many approaches which can be successfully applied to periodic problems fail to be useful for almost periodic problems. Also some concepts and notions introduced for periodic problems cannot be adopted to almost periodic problems directly and need to be adapted from various new points of view.

In [58], the author of the current paper introduced a notion of traveling waves of (1.1) in the case $N=1$, based on the observation that in general the wave front depends on both the time and the space position and that the wave speed is also time dependent. Roughly speaking, a traveling wave solution of (1.1) when $N=1$ is a solution of form

$$
u(t, x)=U(x-c(t), t, c(t)),
$$

where $U(x, t, y)$ is periodic in $y$ and recurrent in $t$ (see Definition A.3 in Appendix A for recurrent functions) $(U(\cdot, t, y)$ can be viewed as a wave front at time $t$ and space position $y)$. We will call $\left[c_{\text {inf }}, c_{\text {sup }}\right]$ its averaged speed interval, where $c_{\text {inf }}=$ $\liminf _{t \rightarrow \infty} \frac{c(t)}{t}$ and $c_{\text {sup }}=\limsup _{t \rightarrow \infty} \frac{c(t)}{t}$ (see Definition 3.4 for details). It should be pointed out that in the time almost periodic case, $c^{\prime}(t)$ may not be a constant (see [55, [56]). This indicates some essential difference between periodic and almost periodic problems. The reader is referred to [55], [56, [57, [59] for the study of traveling wave solutions in space independent and time almost periodic problems. We remark that a notion of traveling wave solutions in time independent and space recurrent problems was introduced in [40] by Matano. The reader is refereed to [1, [7, 22], 44, 66], [70, etc. for the notion of periodic traveling wave solutions.

In 21, the author of the current paper with Huang introduced the notion of a spreading speed interval of (1.1) in the direction of a given unit vector $\xi \in S^{N-1}:=$ $\left\{\xi \in \mathbb{R}^{N} \mid\|\xi\|=1\right\}$ based on the natural features of the classical spreading speeds. Denote $u\left(t, x ; s, y, u_{0}\right)$ as the solution of the following space shifted equation of (1.1):

$$
\frac{\partial u}{\partial t}=\Delta u+\sum_{i=1}^{N} a_{i}(t, x+y) \frac{\partial u}{\partial x_{i}}+f(t, x+y, u), \quad x \in \mathbb{R}^{N},
$$

with initial condition $u\left(s, x ; s, y, u_{0}\right)=u_{0}(x)$ for $u_{0} \in X:=C_{\text {unif }}^{b}\left(\mathbb{R}^{N}, \mathbb{R}\right)$ (see (3.1)), where $y \in \mathbb{R}^{N}$. Put $u\left(t, x ; s, u_{0}\right):=u\left(t, x ; s, 0, u_{0}\right)$. Let

$$
u_{\text {inf }}^{+}=\inf _{t \in \mathbb{R}, x \in \mathbb{R}^{N}} u^{+}(t, x)
$$

(the reader is referred to section 2 for various other notation in the rest of this section). The spreading speed interval $\left[c_{\mathrm{inf}}^{*}(\xi), c_{\mathrm{sup}}^{*}(\xi)\right]$ of (1.1) in the direction of $\xi$ is roughly defined as follows (see Definition 3.2 for details): $c_{\mathrm{inf}}^{*}(\xi)$ is the supremum of all $c \in \mathbb{R}$ satisfying the fact that for any $0 \leq u_{0}<u_{\text {inf }}^{+}\left(u_{0} \in X\right)$ with $0<\liminf _{x \cdot \xi \rightarrow-\infty} u_{0}(x)<u_{\text {inf }}^{+}$(see (3.8) for the notation $\lim _{x \cdot \xi \rightarrow-\infty}$ ) and $u_{0}(x)=0$ for $x \cdot \xi \gg 1$,

$$
\liminf _{x \cdot \xi \leq c t, t \rightarrow \infty}\left(u\left(t+s, x ; s, u_{0}\right)-u^{+}(t+s, x)\right)=0
$$


uniformly in $s \in \mathbb{R}$ (see (3.10) for the notation $\liminf x \cdot \xi \leq c t, t \rightarrow \infty)$ and $c_{\text {sup }}^{*}(\xi)$ is the infimum of all $c \in \mathbb{R}$ satisfying the fact that for any $0 \leq u_{0}<u_{\text {inf }}^{+}\left(u_{0} \in X\right)$ with $0<\liminf _{x \cdot \xi \rightarrow-\infty} u_{0}(x)<u_{\text {inf }}^{+}$and $u_{0}(x)=0$ for $x \cdot \xi \gg 1$,

$$
\limsup _{x \cdot \xi \geq c t, t \rightarrow \infty} u\left(t+s, x ; s, u_{0}\right)=0
$$

uniformly in $s \in \mathbb{R}$ (see (3.11) for the notation $\lim \sup _{x \cdot \xi \geq c t, t \rightarrow \infty}$ ).

We call $u_{0} \in X$ a front or $u\left(t, x ; s, y, u_{0}\right)$ with $u_{0} \in X$ a front solution in the direction of $\xi \in S^{N-1}$ if $u_{0} \geq 0, \liminf _{x \cdot \xi \rightarrow-\infty} u_{0}(x)>0$, and $\lim \sup _{x \cdot \xi \rightarrow \infty} u_{0}(x)=$ 0 (see (3.9) for the notation lim $\sup _{x \cdot \xi \rightarrow \infty}$ ).

The authors of [21] also introduced a notion of generalized propagating speed interval $\left[c_{\text {inf }}\left(u_{0}, \xi\right), c_{\sup }\left(u_{0}, \xi\right)\right]$ of a front $u_{0}$ or a front solution $u\left(t, x ; s, y, u_{0}\right)$ in the direction of $\xi$ (see Definition 3.3). Roughly, $c_{\text {inf }}\left(u_{0}, \xi\right)$ is the supremum of all $c$ such that

$$
\liminf _{x \cdot \xi \leq c t, t \rightarrow \infty}\left(u\left(t+s, x ; s, y, u_{0}\right)-u^{+}(t+s, x+y)\right)=0
$$

uniformly in $s \in \mathbb{R}$ and $y \in \mathbb{R}^{N}$, and $c_{\text {sup }}\left(u_{0}, \xi\right)$ is the infimum of all $c$ such that

$$
\limsup _{x \cdot \xi \geq c t, t \rightarrow \infty} u\left(t+s, x ; s, y, u_{0}\right)=0
$$

uniformly in $s \in \mathbb{R}$ and $y \in \mathbb{R}^{N}$. If $c_{\text {inf }}\left(u_{0}, \xi\right)=c_{\text {sup }}\left(u_{0}, \xi\right), c:=c_{\text {inf }}\left(u_{0}, \xi\right)$ is called the (generalized propagating) speed of the front solution $u\left(t, x ; s, y, u_{0}\right)$ in the direction of $\xi$.

It is shown in 21] that the spreading speed and generalized propagating speed intervals satisfy the following fundamental properties:

- (Boundedness, Proposition [3.5) $\left[c_{\text {inf }}^{*}(\xi), c_{\text {sup }}^{*}(\xi)\right]$ is a finite interval for any $\xi \in S^{N-1}$.

- (Recovery of the classical spreading speed, Proposition 3.5) If (1.1) is periodic in $t$, then $c_{\text {inf }}^{*}(\xi)=c_{\text {sup }}^{*}(\xi)=c^{*}(\xi)$ for any $\xi \in S^{N-1}$.

- (Minimality, Proposition 3.5) $c_{\mathrm{inf}}^{*}(\xi) \leq c_{\mathrm{inf}}\left(u_{0}, \xi\right)$ and $c_{\mathrm{sup}}^{*}(\xi) \leq c_{\mathrm{sup}}\left(u_{0}, \xi\right)$ for any $\xi \in S^{N-1}$ and any front $u_{0} \geq 0$ in the direction of $\xi$.

- (Minimality, Proposition 3.5) If $\left[c_{\mathrm{inf}}(\xi), c_{\mathrm{sup}}(\xi)\right]$ is the averaged speed interval of some traveling wave solution of (1.1) connecting $u^{+}$and $u^{-}$and propagating in the direction of $\xi$ (see Definition 3.4 for a definition), then $c_{\mathrm{inf}}^{*}(\xi) \leq c_{\mathrm{inf}}(\xi)$ and $c_{\text {sup }}^{*}(\xi) \leq c_{\text {sup }}(\xi)$.

- (Spatial spread, Proposition 3.6) If $c>\sup _{\|\xi\|=1} c_{\text {sup }}^{*}(\xi)$, then for any $0 \leq u_{0}<$ $\inf _{t \in \mathbb{R}, x \in \mathbb{R}^{N}} u^{+}(t, x)\left(u_{0} \in X\right)$ with $u_{0}(x)=0$ for $\|x\| \gg 1$,

$$
\limsup _{\|x\| \geq c t, t \rightarrow \infty} u\left(t+s, x ; s, y, u_{0}\right)=0
$$

uniformly in $s \in \mathbb{R}$ and $y \in \mathbb{R}^{N}$ (see (3.12) for the notation $\lim \sup _{\|x\| \geq c t, t \rightarrow \infty}$ ).

- (Spatial spread, Proposition [3.6) If $0<c<\min \left\{c_{\mathrm{inf}}^{*}(\xi), c_{\mathrm{inf}}^{*}(-\xi)\right\}$, then for any $0 \leq u_{0}\left(u_{0} \in X\right)$ with $\inf _{|x \cdot \xi| \leq O(1)} u_{0}(x)>0$,

$$
\liminf _{|x \cdot \xi| \leq c t, t \rightarrow \infty}\left(u\left(t+s, x ; s, y, u_{0}\right)-u^{+}(t+s, x+y)\right)=0
$$

uniformly in $s \in \mathbb{R}$ and $y \in \mathbb{R}^{N}$ (see (3.13) for the notation $\liminf \operatorname{in}_{|x \cdot \xi| \leq c t, t \rightarrow \infty}$ ).

It is seen from the above properties that the concept of spreading speed intervals introduced in [21] is the natural extension of the spreading speeds for time independent or periodic KPP models. It will be seen that the concept of generalized 
propagating speed intervals is the generalization of traveling wave speeds of time independent or periodic KPP models.

The work 21] provides some theoretical foundation for the investigation of spatial spread and front propagation dynamics in time almost periodic KPP models. It is of great mathematical and biological importance to gain some further qualitative and quantitative understanding of spreading and generalized propagating speeds for such KPP models.

In this paper, we develop a variety of important properties of spreading and generalized propagating speeds for time almost periodic and space periodic KPP models. In particular, utilizing the principal Lyapunov exponent and principal Floquet bundle theory for time almost periodic parabolic equations (see [24], 25], 26], [27, 28, 41], 42, and the references therein for the study of principal Lyapunov exponents and principal Floquet bundles for time almost periodic as well as general time dependent parabolic equations), we provide various useful estimates for the spreading and generalized propagating speeds. We establish a variational principle for the spreading speeds and show the existence of a family of front solutions of constant speeds under the so-called linear determinacy condition. We also study the influence of time and space variations on the spreading speeds.

To be more precise, for a given globally Hölder continuous function $a_{0}(t, x)$ which is uniformly almost periodic in $t$ and periodic in $x_{j}$ with period $p_{j}(j=1,2, \cdots, N)$, let $\lambda\left(\mu, \xi ; a_{0}\right)$ be the principal Lyapunov exponent (see Definition 2.6) of

$$
\begin{cases}\frac{\partial u}{\partial t}=\Delta u+\sum_{i=1}^{N} a_{i}^{\mu, \xi}(t, x) \frac{\partial u}{\partial x_{i}}+a_{0}^{\mu, \xi}(t, x) u, & x \in \mathbb{R}^{N}, \\ u\left(t, x_{1}, \cdots, x_{j-1}, x_{j}+p_{j}, x_{j+1}, \cdots, x_{N}\right) & \\ \quad=u\left(t, x_{1}, \cdots, x_{j-1}, x_{j}, x_{j+1}, \cdots, x_{N}\right), & j=1,2, \cdots, N,\end{cases}
$$

where, as in (1.4), $a_{i}^{\mu, \xi}(t, x)=a_{i}(t, x)-2 \mu \xi_{i}(i=1,2, \cdots, N), a_{0}^{\mu, \xi}(t, x)=a_{0}(t, x)-$ $\mu \sum_{i=1}^{N} a_{i}(t, x) \xi_{i}+\mu^{2}$. Observe that the principal Lyapunov exponent of (1.6) is the analog of the principal eigenvalues of elliptic and periodic parabolic problems (see section 2 for basic properties of principal Lyapunov exponents of almost periodic parabolic equations). Put

$$
c_{l}\left(\xi ; a_{0}\right):=\inf _{\mu>0} \frac{\lambda\left(\mu, \xi ; a_{0}\right)}{\mu}
$$

and

$$
c_{l}(\xi):=c_{l}\left(\xi ; a_{0}\right) \quad \text { if } \quad a_{0}(t, x)=f_{u}(t, x, 0):=\frac{\partial f}{\partial u}(t, x, 0),
$$

where $\xi \in S^{N-1}$. We call $c_{l}\left(\xi ; a_{0}\right)$ and $c_{l}(\xi)$ the spreading speed of the linear equation (1.6) and the linear spreading speed of (1.1) in the direction of $\xi \in S^{N-1}$, respectively.

Among others, the following properties are proved in this paper.

- (Upper bound for spreading speeds in the partially spatially homogeneous case, Theorem 4.1) Assume that $a_{i}(t, x) \equiv a_{i}(t, 0)(i=1,2, \cdots, N)$ and that there is a globally Hölder continuous almost periodic function $a_{0}(\cdot)$ such that $f(t, x, u) \leq$ $a_{0}(t) u$ for $t \in \mathbb{R}, x \in \mathbb{R}^{N}, u \geq 0$. Then

$$
c_{\mathrm{sup}}^{*}(\xi) \leq c_{l}\left(\xi ; a_{0}\right) \quad \text { for any } \quad \xi \in S^{N-1} .
$$

(See Theorem 5.1 for some general cases.) 
- (Lower bound for spreading speeds in the partially spatially homogeneous case, Theorem 4.11) Assume that $a_{i}(t, x) \equiv a_{i}(t, 0)(i=1,2, \cdots, N)$ and that there is a globally Hölder continuous almost periodic function $a_{0}(\cdot)$ such that $\lambda\left(0, \xi ; a_{0}\right)>0$ for $\xi \in S^{N-1}\left(\lambda\left(0, \xi ; a_{0}\right)\right.$ is independent of $\left.\xi \in S^{N-1}\right)$ and that $f(t, x, u) \geq a_{0}(t) u$ for $t \in \mathbb{R}, x \in \mathbb{R}^{N}, 0 \leq u \ll 1$. Then

$$
c_{\text {inf }}^{*}(\xi) \geq c_{l}\left(\xi ; a_{0}\right) \quad \text { for any } \quad \xi \in S^{N-1} .
$$

In particular, if $a_{i}(t, x) \equiv a_{i}(t, 0)(i=1,2, \cdots, N)$ and $f_{u}(t, x, 0) \equiv f_{u}(t, 0,0)$, then

$$
c_{\text {inf }}^{*}(\xi) \geq c_{l}(\xi) \quad \text { for any } \xi \in S^{N-1} \text {. }
$$

(See Theorem 5.1 for some general cases.)

- Variational principle for spreading speeds in the partially spatially homogeneous case, Theorem 4.1. Assume that $a_{i}(t, x) \equiv a_{i}(t, 0)(i=1,2, \cdots, N)$, $f(t, u) \leq f_{u}(t, x, 0) u$ for $t \in \mathbb{R}, x \in \mathbb{R}^{N}$, and $u \geq 0$, and $f_{u}(t, x, 0) \equiv f_{u}(t, 0,0)$. Then

$$
c_{\text {inf }}^{*}(\xi)=c_{\text {sup }}^{*}(\xi)=c_{l}(\xi) \quad \text { for any } \xi \in S^{N-1} .
$$

(See Theorem 5.1 for some general cases.)

- (Front solutions of constant speeds in the partially spatially homogeneous case, Theorem 4.2) Assume that $a_{i}(t, x) \equiv a_{i}(t, 0)(i=1,2, \cdots, N), f(t, x, u) \leq$ $f_{u}(t, x, 0) u$ for $t \in \mathbb{R}, x \in \mathbb{R}^{N}$, and $u \geq 0$, and $f_{u}(t, x, 0) \equiv f_{u}(t, 0,0)$. If $\xi \in S^{N-1}$ and $u_{0} \geq 0\left(u_{0} \in X\right)$ are such that $\lim _{x \cdot \xi \rightarrow-\infty} u_{0}(x \cdot \xi)>0$ and $C_{1} e^{-\mu x \cdot \xi} \leq u_{0}(x) \leq$ $C_{2} e^{-\mu x \cdot \xi}$ for $x \cdot \xi \gg 1$ and some $C_{1}, C_{2}>0$ and $0<\mu<\mu^{*}$, where $\mu^{*}$ is such that $\frac{\lambda\left(\mu^{*}, \xi ; a_{0}\right)}{\mu^{*}}=c_{l}\left(\xi ; a_{0}\right)$ with $a_{0}(t)=f_{u}(t, 0,0)$, then

$$
c_{\text {inf }}\left(u_{0}, \xi\right)=u_{\text {sup }}\left(u_{0}, \xi\right)=\frac{\lambda\left(\mu, \xi ; a_{0}\right)}{\mu} .
$$

Hence for any $c \geq c_{l}(\xi)$, there is a front solution of (1.1) in the direction of $\xi$ with speed $c$ and there are no front solutions of slower speed in the direction of $\xi$. (See Theorem 5.2 for some general cases.)

As in time independent and periodic cases, we call the condition $f(t, x, u) \leq$ $f_{u}(t, x, 0) u$ for $t \in \mathbb{R}, x \in \mathbb{R}^{N}$ and $u \geq 0$ the linear determinacy condition.

The properties stated above extend many classical spatial spread and front propagation properties (e.g., the variational principle for the spreading speed, the existence of traveling wave solutions of speed greater than or equal to the spreading speed, and the nonexistence of traveling wave solutions of slower speed) for time independent or periodic KPP models to time almost periodic ones. However, it remains open whether in general time almost periodic KPP models there are traveling wave solutions with averaged speed greater than or equal to $c_{\mathrm{inf}}^{*}(\xi)=c_{\mathrm{sup}}^{*}(\xi)$ in a given direction of $\xi \in S^{N-1}$, provided that $c_{\mathrm{inf}}^{*}(\xi)=c_{\mathrm{sup}}^{*}(\xi)$. It also remains open whether in general time almost KPP models, $c_{\mathrm{inf}}^{*}(\xi)=c_{\mathrm{sup}}^{*}(\xi)$ for any $\xi \in S^{N-1}$.

In addition to the properties stated above, we also prove the following properties about the influence of space and time variation on the spreading speeds.

- (The influence of space and time variation on linear spreading speed, Theorem 6.1) If $a_{i}(t, x) \equiv a_{i}(0, x)(i=1,2, \cdots, N)$, then

$$
c_{l}\left(\xi ; a_{0}\right) \geq c_{l}\left(\xi ; \hat{a}_{0}\right) \quad \text { for any } \xi \in S^{N-1}
$$

and $c_{l}\left(\xi ; a_{0}\right)=c_{l}\left(\xi ; \hat{a}_{0}\right)$ for some $\xi \in S^{N-1}$ if and only if $a_{0}(t, x)$ is of the form $a_{0}(t, x)=a_{01}(t)+a_{02}(x)$ for some $a_{01}(\cdot)$ and $a_{02}(\cdot)$ and any $t \in \mathbb{R}, x \in \mathbb{R}^{N}$, where 
$a_{0}(t, x)=f_{u}(t, x, 0)$ and $\hat{a}_{0}(x)=\lim _{t-s \rightarrow \infty} \frac{1}{t-s} \int_{s}^{t} a_{0}(\tau, x) d \tau$ (see Theorem A.1 in Appendix A for the existence of this limit). (See Theorem 6.1 for more properties).

- (The influence of space and time variation on spreading speeds, Theorem 6.2) Assume that $a_{i}(t, x) \equiv a_{i}(0, x)(i=1,2, \cdots, N)$ and that $f_{u}(t, x, 0)$ can be approximated by time periodic functions. Then

$$
c_{\mathrm{sup}}^{*}(\xi) \geq c_{\mathrm{inf}}^{*}(\xi) \geq c_{l}\left(\xi ; \hat{a}_{0}\right) \quad \text { for any } \xi \in S^{N-1},
$$

where $a_{0}(t, x)=f_{u}(t, x, 0)$. Furthermore, if $f(t, x, u) \leq f_{u}(t, x, 0) u$ for $t \in \mathbb{R}$, $x \in \mathbb{R}^{N}$, and $u \geq 0$, then

$$
c_{\mathrm{sup}}^{*}(\xi)=c_{\mathrm{inf}}^{*}(\xi) \geq c_{l}\left(\xi ; \hat{a}_{0}\right) \quad \text { for any } \quad \xi \in S^{N-1},
$$

and $c_{\mathrm{inf}}^{*}(\xi)=c_{l}\left(\xi ; \hat{a}_{0}\right)$ for some $\xi \in S^{N-1}$ if and only if $a_{0}(t, x)$ is of the form $a_{0}(t, x)=a_{01}(t)+a_{02}(x)$ for some $a_{01}(\cdot)$ and $a_{02}(\cdot)$ and any $t \in \mathbb{R}, x \in \mathbb{R}^{N}$. (See Theorem 6.2 for more properties.)

Note that $\hat{c}^{*}(\xi):=c_{l}\left(\xi ; \hat{a}_{0}\right):=\inf _{\mu>0} \frac{\lambda\left(\mu, \xi ; \hat{a}_{0}\right)}{\mu}$ is the spreading speed of the following averaged equation of (1.1):

$$
\frac{\partial u}{\partial t}=\Delta u+\sum_{i=1}^{N} a_{i}(x) \frac{\partial u}{\partial x_{i}}+\hat{f}(x, u)
$$

where $a_{i}(x)=a_{i}(0, x)(i=1,2, \cdots, N)$ and $\hat{f}(x, u)=\lim _{t-s \rightarrow \infty} \frac{1}{t-s} \int_{s}^{t} f(\tau, x, u) d \tau$ (see Theorem A.1 in Appendix A for the existence of this limit), provided that $\hat{f}(x, u)$ is of KPP type and $\hat{f}(x, u) \leq \hat{a}_{0} u$ for $x \in \mathbb{R}^{N}$ and $u \geq 0$. Hence the space and time variation of the nonlinearity in KPP models cannot slow down the spatial spread. It indeed speeds up the spatial spread except in the degenerate case where $f_{u}(t, x, 0)$ is of form $f_{u}(t, x, 0)=a_{01}(t)+a_{02}(x)$, which is new even for time periodic KPP models. It should be pointed out that in [7] the influence of the space variation of the nonlinearity in some time independent KPP models is studied. It should also be pointed out that in [45], 48, [52, 75] the influence of space and time variation of some periodic shear flows on the spreading speed of KPP models is studied.

The rest of the paper is organized as follows. In section 2 we develop some principal Lyapunov exponents and principal Floquet bundle theory for time almost periodic and space periodic linear parabolic equations, which is of great interest in its own and is among the main tools for the proofs of the theorems in later sections. In section 3 we introduce the notation and definitions, and we present some fundamental results proved in 21] to be used in later sections. In section 4 we study spreading and generalized propagating speeds in the partially spatially homogeneous case. Section 5 is devoted to the investigation of spreading and generalized propagating speeds in some general case. We explore the influence of space and time variation on spreading speeds in section 6 . The paper concludes with an appendix on compact flows and almost periodic functions.

We finish the introduction with the following remark. The concepts and many results established in this paper can be applied to the following more general time dependent KPP models:

$$
\frac{\partial u}{\partial t}=\sum_{i, j=1}^{N} a_{i j}(t, x) \frac{\partial^{2} u}{\partial x_{i} \partial x_{j}}+\sum_{i=1}^{N} a_{i}(t, x) \frac{\partial u}{\partial x_{i}}+f(t, x, u), \quad x \in \mathbb{R}^{N},
$$


where $a_{i j}=a_{j i}$ satisfies the ellipticity condition; i.e., there is $\alpha_{0}>0$ such that

$$
\sum_{i, j=1}^{N} a_{i j}(t, x) \xi_{i} \xi_{j} \geq \alpha_{0}|\xi|^{2} \quad \text { for all } \quad(t, x) \in \mathbb{R}^{N+1}, \quad \xi \in \mathbb{R}^{N},
$$

and $f$ is of KPP type in proper sense.

\section{SPECTRAL THEORY FOR LINEAR ALMOST PERIODIC PARABOLIC EQUATIONS WITH PERIODIC BOUNDARY CONDITIONS}

In this section, we develop the needed principal spectral theory for time almost periodic parabolic equations with periodic boundary conditions. Such a theory is an extension of the principal eigenvalue theory for elliptic and periodic parabolic problems. It is of great interest on its own and can be established by the arguments similar to those used in 24, 25, 26, 27, 28, 41, 42, 49 for the establishment of principal spectral theory for almost periodic parabolic equations complemented with Dirichlet or Neumann boundary conditions. We will therefore only mention the references for the proofs of those results which can be proved by the arguments in the references.

This section is independent of other sections. The theories developed in this section are among the main tools for the proofs of the theorems in other sections.

We first consider a family of linear parabolic equations of the form

$$
u_{t}=\Delta u+\sum_{i=1}^{N} b_{i}(t, x) \frac{\partial u}{\partial x_{i}}+b_{0}(t, x), \quad x \in \mathbb{R}^{N},
$$

complemented with the periodic boundary condition

$$
\begin{aligned}
& u\left(t, x_{1}, \cdots, x_{j-1}, x_{j}+p_{j}, x_{j+1}, \cdots, x_{N}\right) \\
& \quad=u\left(t, x_{1}, \cdots, x_{j-1}, x_{j}, x_{j+1}, \cdots, x_{N}\right), \quad j=1,2, \cdots, N,
\end{aligned}
$$

where $p_{j}>0(j=1,2, \cdots, N), b:=\left(b_{i}, b_{0}\right):=\left(\left\{b_{i}\right\}_{i=1}^{N}, b_{0}\right) \in Y$, and $Y$ is a subset of $C\left(\mathbb{R} \times \mathbb{R}^{N}, \mathbb{R}^{N+1}\right)$. To emphasis the dependence of (2.1) on $b$, we may write it as $(2.1)_{b}$.

We make the following standard assumption on $Y$.

$(\mathbf{H}-\mathbf{Y})$ For any $b=\left(b_{i}, b_{0}\right) \in Y, b_{i}(t, x)(i=1,2, \cdots, N)$ and $b_{0}(t, x)$ are uniformly almost periodic in $t$ and periodic in $x_{j}$ with period $p_{j}>0(j=1,2, \cdots, N)$ and are globally Hölder continuous in $t, x$. Moreover, $Y$ is translation invariant in $t$ (i.e. for any $b \in Y$ and $t \in \mathbb{R}, \sigma_{t} b:=b \cdot t:=b(t+\cdot, \cdot) \in Y$ ) and is connected and compact under uniform convergence topology.

In the following, we assume that $Y$ satisfies $(\mathrm{H}-\mathrm{Y})$ and $Y$ is equipped with the uniform convergence topology. Then $\left(Y,\left(\sigma_{t}\right)_{t \in \mathbb{R}}\right)$ is a compact flow. We say $Y$ is unique ergodic if $\left(Y,\left(\sigma_{t}\right)_{t \in \mathbb{R}}\right)$ is unique ergodic (i.e. $\left(Y,\left(\sigma_{t}\right)_{t \in \mathbb{R}}\right)$ has a unique invariant measure; see Definition A.1 for the definition of unique ergodicity of a compact flow). For a given Banach space $X,\|\cdot\|_{X}$ denotes the norm in $X$.

Let

$$
\begin{gathered}
X_{L}=\left\{u \in C\left(\mathbb{R}^{N}, \mathbb{R}\right) \mid u \quad \text { is periodic in } x_{j} \text { with period } p_{j}\right. \\
\text { for } j=1,2, \cdots, N\}
\end{gathered}
$$


be equipped with uniform convergence topology. Let $\mathcal{L}\left(X_{L}, X_{L}\right)$ be the space of bounded linear operators from $X_{L}$ to $X_{L}$. Let

$$
X_{L}^{+}=\left\{u \in X_{L} \mid u(x) \geq 0, x \in \mathbb{R}^{N}\right\}
$$

and

$$
\operatorname{Int}\left(X_{L}^{+}\right):=X_{L}^{++}=\left\{u \in X_{L}^{+} \mid u(x)>0, x \in \mathbb{R}^{N}\right\} .
$$

For $u_{1}, u_{2} \in X_{L}$, we write

$$
u_{1} \leq u_{2} \quad\left(u_{1} \geq u_{2}\right) \quad \text { if } \quad u_{2}-u_{1} \in X_{L}^{+} \quad\left(u_{1}-u_{2} \in X_{L}^{+}\right)
$$

and

$$
u_{1} \ll u_{2} \quad\left(u_{1} \gg u_{2}\right) \quad \text { if } \quad u_{2}-u_{1} \in X_{L}^{++} \quad\left(u_{1}-u_{2} \in X_{L}^{++}\right) .
$$

Let

$$
X_{\text {per }}^{1}\left(\mathbb{R}^{N}\right)=\left\{u \in X_{L} \mid \frac{\partial u}{\partial x_{i}} \in X_{L}, i=1,2, \cdots, N\right\} .
$$

It follows from [20] that $-\Delta$ is a sectorial operator on $X_{L}$; denote it by $-\left.\Delta\right|_{X_{L}}$. Let $X_{L}^{\alpha}$ be the fractional power space of $-\Delta$ on $X_{L}(0 \leq \alpha \leq 1)$. Note that $X_{L}^{0}=$ $X_{L}$ and $X_{L}^{1}=\mathcal{D}\left(-\left.\Delta\right|_{X_{L}}\right)$. Let $0<\alpha_{0}<1$ be such that $X_{L}^{\alpha_{0}}$ is compactly imbedded into $C_{\text {per }}^{1}\left(\mathbb{R}^{N}\right)$. Then by [20], for any $b \in Y$ and $u_{0} \in X_{L}^{\alpha_{0}}$ there is a unique solution $u\left(t, \cdot ; u_{0}, b\right) \in X_{L}^{\alpha_{0}}$ of (2.1) and (2.2) with initial condition $u\left(0, \cdot ; u_{0}, b\right)=u_{0}(\cdot)$. Put $U(t, b) u_{0}:=u\left(t, \cdot ; u_{0}, b\right)$ for $u_{0} \in X_{L}^{\alpha_{0}}$. Following from the results in [20] and classical theory for parabolic equations, we have

Theorem 2.1. (1) (Joint continuity). The map $\left[[0, \infty) \times X_{L}^{\alpha_{0}} \times Y \ni\left(t, u_{0}, b\right) \mapsto\right.$ $\left.U(t, b) u_{0} \in X_{L}^{\alpha_{0}}\right]$ is continuous.

(2) (Norm continuity). For any $t \geq 0$, the $\operatorname{map}\left[Y \ni b \mapsto U(t, b) \in \mathcal{L}\left(X_{L}^{\alpha_{0}}, X_{L}^{\alpha_{0}}\right)\right]$ is continuous.

(3) (Strong monotonicity). For any $t>0$ and $b \in Y, U(t, b)$ is strongly monotone in the sense that if $u_{1}, u_{2} \in X_{L}^{\alpha_{0}}$ and $u_{1} \leq u_{2}, u_{1} \neq u_{2}$, then $U(t, b) u_{1} \ll U(t, b) u_{2}$ for any $t>0$ and $b \in Y$.

(4) (Compactness). For any $t>0, U(t, \cdot)$ is compact in the sense that for any bounded set $E \subset X_{L}^{\alpha_{0}},\left\{U(t, b) u_{0} \mid b \in Y, u_{0} \in E\right\}$ is a relatively compact subset of $X_{L}^{\alpha_{0}}$.

By Theorem 2.1, (2.1) and (2.2) generate a skew-product semiflow on $X_{L}^{\alpha_{0}} \times Y$ :

$$
\begin{gathered}
\Pi_{t}: X_{L}^{\alpha_{0}} \times Y \rightarrow X_{L}^{\alpha_{0}} \times Y, \quad t \geq 0, \\
\Pi_{t}\left(u_{0}, b\right)=\left(U(t, b) u_{0}, b \cdot t\right) .
\end{gathered}
$$

The following theorem follows from [50] (see also [41, 42], 60]).

Theorem 2.2 (Exponential separation). There are subspaces $X_{L}^{\alpha_{0}, 1}(b), X_{L}^{\alpha_{0}, 2}(b)$ of $X_{L}^{\alpha_{0}}$ such that $X_{L}^{\alpha_{0}, 1}(b), X_{L}^{\alpha_{0}, 2}(b)$ are continuous in $b \in Y$, and satisfy the following properties:

(1) $X_{L}^{\alpha_{0}}=X_{L}^{\alpha_{0}, 1}(b) \oplus X_{L}^{\alpha_{0}, 2}(b)$ for any $b \in Y$.

(2) $X_{L}^{\alpha_{0}, 1}(b)=\operatorname{Span}\{\tilde{\phi}(b)\}, \tilde{\phi}(b) \in \operatorname{Int}\left(X_{L}^{\alpha_{0}} \cap X_{L}^{+}\right)$and is continuous in $b$, and $\|\tilde{\phi}(b)\|_{X_{L}^{\alpha_{0}}}=1$ for any $b \in Y$.

(3) $X_{L}^{\alpha_{0}, 2}(b) \cap \operatorname{Int}\left(X_{L}^{\alpha_{0}} \cap X_{L}^{+}\right)=\emptyset$ for any $b \in Y$.

(4) $U(t, b) X_{L}^{\alpha_{0}, 1}(b)=X_{L}^{\alpha_{0}, 1}\left(\sigma_{t} b\right)$ and $U(t, b) X_{L}^{\alpha_{0}, 2}(b) \subset X_{L}^{\alpha_{0}, 2}\left(\sigma_{t} b\right)$ for any $b \in$ $Y$ and $t>0$. 
(5) There are $M, \gamma>0$ such that

$$
\frac{\|U(t, b) \tilde{\phi}(b)\|_{X_{L}^{\alpha_{0}}}}{\|U(t, b) w\|_{X_{L}^{\alpha_{0}}}} \leq M e^{-\gamma t}
$$

for any $t>0, b \in Y$, and $w \in X_{L}^{\alpha_{0}, 2}(b)$ with $\|w\|_{X_{L}^{\alpha_{0}}}=1$.

By classical theory for parabolic equations and the continuity of $\tilde{\phi}(b)$ in $b \in Y$ with respect to the $\|\cdot\|_{\alpha_{0}}$-norm, there are constants $C_{1}, C_{2}>0$ such that

$$
\frac{1}{C_{1}}\|\tilde{\phi}(b)\|_{X_{L}^{\alpha_{0}}} \leq\|\tilde{\phi}(b)\|_{L_{2}(D)} \leq C_{1}\|\tilde{\phi}(b)\|_{X_{L}^{\alpha_{0}}}
$$

and

$$
\frac{1}{C_{2}}\|\tilde{\phi}(b)\|_{X_{L}^{\alpha_{0}}} \leq\|\tilde{\phi}(b)\|_{X_{L}} \leq C_{2}\|\tilde{\phi}(b)\|_{X_{L}^{\alpha_{0}}}
$$

for any $b \in Y$.

Let

$$
\phi(b)=\tilde{\phi}(b) /\|\tilde{\phi}(b)\|_{L_{2}(D)} .
$$
Let

Let $D=\left[0, p_{1}\right] \times\left[0, p_{2}\right] \times \cdots \times\left[0, p_{N}\right]$ and $\langle\cdot, \cdot\rangle$ be the inner product in $L_{2}(D)$.

$$
\kappa(b)=\left\langle\Delta \phi(b)+\sum_{i=1}^{N} b_{i}(t, x) \frac{\partial \phi(b)}{\partial x_{i}}+b_{0} \phi(b), \phi(b)\right\rangle .
$$

Theorem 2.3. $\kappa(b)$ is continuous in $b \in Y$.

Proof. This follows from Theorem 2.2 and the following fact:

$$
\kappa(b)=-\langle\nabla \phi(b), \nabla \phi(b)\rangle+\left\langle\sum_{i=1}^{N} b_{i}(t, x) \frac{\partial \phi(b)}{\partial x_{i}}+b_{0} \phi(b), \phi(b)\right\rangle .
$$

Let

$$
\eta(t, b)=\|U(t, b) \phi(b)\|_{L_{2}(D)}
$$

Then we have

$$
\eta_{t}(t, b)=\kappa\left(\sigma_{t} b\right) \eta(t, b)
$$

therefore

$$
\eta(t, b)=e^{\int_{0}^{t} \kappa\left(\sigma_{\tau} b\right) d \tau} .
$$

Let

$$
v(t, x ; b)=\frac{U(t, b) \phi(b)}{\|U(t, b) \phi(b)\|_{L_{2}}} \equiv \phi\left(\sigma_{t} b\right)(x) .
$$

We have that $v(t, x ; b)$ satisfies

$$
v_{t}=\Delta v+\sum_{i=1}^{N} b_{i}(t, x) \frac{\partial v}{\partial x_{i}}+b_{0}(t, x) v-\kappa\left(\sigma_{t} b\right) v, \quad x \in \mathbb{R}^{N} .
$$

Theorem 2.4. If $Y$ is unique ergodic, then

$$
\lim _{t-s \rightarrow \infty} \frac{1}{t-s} \ln \left\|U\left(t-s, \sigma_{s} b\right)\right\|_{\alpha_{0}}=\lim _{t-s \rightarrow \infty} \frac{1}{t-s} \int_{s}^{t} \kappa\left(\sigma_{\tau} b\right) d \tau
$$

for all $b \in Y$, and the limit is independent of $b$. 
Proof. It follows from Theorems 2.2 and 2.3. and the results in [29].

Definition 2.5. Assume that $Y$ is unique ergodic. $\lambda(Y)$ is called the principal Lyapunov exponent of (2.1) and (2.2), where

$$
\lambda(Y)=\lim _{t-s \rightarrow \infty} \frac{1}{t-s} \ln \left\|U\left(t-s, \sigma_{s} b\right)\right\|_{\alpha_{0}}
$$

for any $b \in Y$.

We now consider a single linear parabolic equation

$$
u_{t}=\Delta u+\sum_{i=1}^{n} a_{i}(t, x) \frac{\partial u}{\partial x_{i}}+a_{0}(t, x) u, \quad x \in \mathbb{R}^{N},
$$

complemented with the periodic boundary condition (2.2), where $a_{i}(t, x)(i=$ $1,2, \cdots, N)$ and $a_{0}(t, x)$ are uniformly almost periodic in $t$ and periodic in $x_{j}$ with period $p_{j}(j=1,2, \cdots, N)$, and are globally Hölder continuous in $t, x$.

For any $\mu \in \mathbb{R}$ and $\xi \in S^{N-1}$, also consider

$$
u_{t}=\Delta u+\sum_{i=1}^{N} a_{i}^{\mu, \xi} \frac{\partial u}{\partial x_{i}}+a_{0}^{\mu, \xi}(t, x) u, \quad x \in \mathbb{R}^{N},
$$

complemented with the periodic boundary condition (2.2), where $a_{i}^{\mu, \xi}=a_{i}-$ $2 \mu \xi_{i}, \quad i=1,2, \cdots, N$ and $a_{0}^{\mu, \xi}=a_{0}-\mu \sum_{i=1}^{N} a_{i} \xi_{i}+\mu^{2}$. Note that if $\mu=0$, then $a_{i}^{\mu, \xi}=a_{i}$ and $a_{0}^{\mu, \xi}=a_{0}$ for any $\xi \in S^{N-1}$.

Let $a:=\left(a_{i}, a_{0}\right):=\left(\left\{a_{i}\right\}_{i=1}^{N}, a_{0}\right)$ and

$$
Y(a)=\operatorname{cl}\{a \cdot s \mid s \in \mathbb{R}\},
$$

where $a \cdot s(t, x):=\sigma_{s} a(t, x):=a(t+s, x)$ and the closure is taken under the open compact topology. Then (H-Y) is satisfied with $Y=Y(a)$. Hence for any $u_{0} \in X_{L}^{\alpha_{0}}$ and any $s \in \mathbb{R}$, (2.7) and (2.2) have a unique classical solution $u\left(t, x ; s, u_{0}, a\right)$ with initial condition $u\left(s, x ; s, u_{0}, a\right)=u_{0}(x)$.

Similarly, let

$$
Y\left(a^{\mu, \xi}\right)=\operatorname{cl}\left\{a^{\mu, \xi} \cdot s \mid s \in \mathbb{R}\right\},
$$

where $a^{\mu, \xi}=\left(a_{i}^{\mu, \xi}, a_{0}^{\mu, \xi}\right):=\left(\left\{a_{i}^{\mu, \xi}\right\}_{i=1}^{N}, a_{0}^{\mu, \xi}\right), a^{\mu, \xi} \cdot s:=\sigma_{s} a^{\mu, \xi}:=a^{\mu, \xi}(\cdot+s, \cdot)$, and the closure is taken under open compact topology. Then $Y\left(a^{\mu, \xi}\right)$ satisfies (H-Y) with $Y$ being replaced by $Y\left(a^{\mu, \xi}\right)$. Hence (2.8) and (2.2) have a unique classical solution $u\left(t, x ; s, u_{0}, a^{\mu, \xi}\right)$ with $u\left(s, x ; s, u_{0}, a^{\mu, \xi}\right)=u_{0}(x)$ for any $u_{0} \in X_{L}^{\alpha_{0}}$.

Note that $Y\left(a^{0, \xi}\right)(=Y(a))$ and $Y\left(a^{\mu, \xi}\right)$ are unique ergodic and minimal for any $\mu \in \mathbb{R}$ and $\xi \in S^{N-1}$ (see Remark A.1 (2)). Theorems 2.2 through 2.4 can then be applied to $Y=Y\left(a^{\mu, \xi}\right)$. Put

$$
\lambda(\mu, \xi ; a):=\lambda\left(a^{\mu, \xi}\right):=\lambda\left(Y\left(a^{\mu, \xi}\right)\right)
$$

and

$$
\phi^{\mu, \xi}(t, \cdot ; a):=\phi\left(\sigma_{t} a^{\mu, \xi}\right)(\cdot) .
$$

Definition 2.6. We call $\lambda(\mu, \xi ; a)$ the principal Lyapunov exponent of (2.8) and (2.2).

Observe that $\lambda(\mu, \xi ; a)$ and $\phi^{\mu, \xi}(t, \cdot ; a)$ are analogs of principal eigenvalues and principal eigenfunctions of elliptic and periodic parabolic problems, respectively. In literature, $\left\{\operatorname{span}\left(\phi\left(\sigma_{t} a^{\mu, \xi}\right)\right)\right\}_{t \in \mathbb{R}}$ is call the principal Floquet bundle of (2.8) associated to the principal Lyapunov exponent. 
Theorem 2.7. (1) $\lambda(\mu, \xi ; a)$ is continuous in $\mu \in \mathbb{R}, \xi \in S^{N-1}$, and a with respect to uniform convergence topology.

(2) Fix a. There is $\beta>0$ such that

$$
\lambda(\mu, \xi ; a) \geq \beta \mu^{2}
$$

for any $\xi \in S^{N-1}$ and $\mu \gg 1$.

(3) Fix a and assume that $\lambda(0, \xi ; a)>0$ (note that $\lambda(0, \xi ; a)$ is independent of $\left.\xi \in S^{N-1}\right)$. There are $\mu_{0}^{-}>0$ and $\mu_{0}^{+}>0$ with $\mu_{0}^{-}<\mu_{0}^{+}$, and $\beta_{0}>0$ such that

$$
\inf _{\mu>0} \frac{\lambda(\mu, \xi ; a)}{\mu}=\inf _{\mu_{0}^{-} \leq \mu \leq \mu_{0}^{+}} \frac{\lambda(\mu, \xi ; a)}{\mu} \leq \beta_{0} \mu_{0}^{+}
$$

for any $\xi \in S^{N-1}$.

Proof. (1) By Theorem 2.4,

$$
\lambda(\mu, \xi ; a)=\lim _{t-s \rightarrow \infty} \frac{1}{t-s} \int_{s}^{t} \kappa\left(\sigma_{\tau} a^{\mu, \xi}\right) d \tau .
$$

It then follows from Theorem 2.3 that $\lambda(\mu, \xi ; a)$ is continuous in $\mu \in \mathbb{R}, \xi \in S^{N-1}$, and $a$ with respect to uniform convergence topology.

(2) Note that

$$
a_{0}^{\mu, \xi}=a_{0}-\mu \sum_{i=1}^{N} b_{i} \xi_{i}+\mu^{2} .
$$

Hence there is $\beta>0$ such that

$$
a_{0}^{\mu, \xi}(t, x) \geq \beta \mu^{2}
$$

for all $t \in \mathbb{R}, x \in \mathbb{R}^{N}, \xi \in S^{N-1}$, and $\mu \gg 1$. Then by comparison principal for parabolic equations,

$$
U\left(t, a^{\mu, \xi}\right) u_{i d} \geq e^{\beta \mu^{2} t}
$$

for $\mu \gg 1$ and $t>0$, where $u_{i d} \equiv 1$. It then follows that

$$
\lambda(\mu, \xi ; a) \geq \beta \mu^{2}
$$

for $\mu \gg 1$ and $\xi \in S^{N-1}$.

(3) By (1) and the assumption $\lambda(0, \xi ; a)>0$, we have

$$
\frac{\lambda(\mu, \xi ; a)}{\mu} \rightarrow \infty
$$

as $\mu \rightarrow 0+$. This together with (2) implies that there are $\mu_{0}^{-}>0, \mu_{0}^{+}$with $\mu_{0}^{-}<\mu_{0}^{+}$, and $\beta_{0}>0$ such that

$$
\inf _{\mu>0} \frac{\lambda(\mu, \xi ; a)}{\mu}=\inf _{\mu_{0}^{-} \leq \mu \leq \mu_{0}^{+}} \frac{\lambda(\mu, \xi ; a)}{\mu} \leq \beta_{0} \mu_{0}^{+} .
$$

Observe that for any $u_{0} \in C\left(\mathbb{R}^{N}, \mathbb{R}\right)$ with

$$
\left|u_{0}(x)\right| \leq C e^{\alpha \sum_{i=1}^{N}\left|x_{i}\right|}
$$


for some $\alpha, C>0$, (2.7) also has a unique solution $u\left(t, x ; s, u_{0}, a\right)$ with $u(s, x$; $\left.s, u_{0}, a\right)=u_{0}(x)$ (see [16]). Regarding solutions of (2.7) without the periodic boundary condition, we have

Theorem 2.8. For any given $\xi \in S^{N-1}$ and $\mu>0$,

$$
u(t, x)=e^{-\mu\left(x \cdot \xi-\frac{\int_{s}^{t} \kappa\left(\sigma_{\tau} a^{\mu, \xi}\right) d \tau}{\mu}\right)} \phi\left(\sigma_{t} a^{\mu, \xi}\right)(x)
$$

is a solution of (2.7).

When $a_{i}$ and $a_{0}$ are periodic in $t$ with period $T$, let $\lambda^{*}(\mu, \xi ; a)$ be the principal eigenvalue of the periodic parabolic eigenvalue problem (1.4) and $\phi^{*}(t, x ; \mu, \xi, a)$ be an associated positive principal eigenfunction. Then

Theorem 2.9. For any given $\xi \in S^{N-1}, \mu>0$,

$$
u(t, x)=e^{-\mu\left(x \cdot \xi-\frac{\lambda^{*}(\mu, \xi ; a) t}{\mu}\right)} \phi^{*}(t, x ; \mu, \xi, a)
$$

is a solution of (2.7).

Note that $\phi\left(\sigma_{t} a^{\mu, \xi}\right)=\frac{\phi^{*}(t, ; ; \mu, \xi, a)}{\left\|\phi^{*}(t, ;, \mu, \xi, a)\right\|_{L_{2}(D)}}$.

The rest of this section is to discuss the influence of the time and/or space variation of $a=\left(a_{i}, a_{0}\right)$ on the principal Lyapunov exponent $\lambda(\mu, \xi ; a)$ of $(2.8)$ and (2.2).

By Theorem A.1, the following limits exist:

$$
\begin{gathered}
\hat{a}_{i}(x):=\lim _{t-s \rightarrow \infty} \frac{1}{t-s} \int_{s}^{t} a_{i}(\tau, x) d \tau, \quad i=1,2, \cdots, N, \\
\hat{a}_{0}(x):=\lim _{t-s \rightarrow \infty} \frac{1}{t-s} \int_{s}^{t} a_{0}(\tau, x) d \tau, \\
\hat{a}_{i}^{\mu, \xi}(x):=\lim _{t-s \rightarrow \infty} \frac{1}{t-s} \int_{s}^{t} a_{i}^{\mu, \xi}(\tau, x) d \tau\left(=\hat{a}_{i}(x)-2 \mu \xi_{i}\right), \quad i=1,2, \cdots, N,
\end{gathered}
$$

and

$$
\hat{a}_{0}^{\mu, \xi}(x):=\lim _{t-s \rightarrow \infty} \frac{1}{t-s} \int_{s}^{t} a_{0}^{\mu, \xi}(\tau, x) d \tau\left(=\hat{a}_{0}(x)-\mu \sum_{i=1}^{N} \hat{a}_{i}(x) \xi+\mu^{2}\right) .
$$

Let $\hat{\lambda}(\mu, \xi ; a)$ be the principal eigenvalue of the following time averaged equation of (2.8),

$$
u_{t}=\Delta u+\sum_{i=1}^{N} \hat{a}_{i}^{\mu, \xi}(x) \frac{\partial u}{\partial x_{i}}+\hat{a}_{0}^{\mu, \xi}(x) u, \quad x \in \mathbb{R}^{N},
$$

together with the boundary condition $(\underline{2.2})$ and $\hat{a}=\left(\hat{a}_{i}, \hat{a}_{0}\right)$.

Let

$$
\begin{gathered}
\check{a}_{i}(t):=\frac{1}{|D|} \int_{D} a_{i}(t, x) d x, \quad i=1,2, \cdots, N, \\
\check{a}_{0}(t):=\frac{1}{|D|} \int_{D} a_{0}(t, x) d x, \\
\check{a}_{i}^{\mu, \xi}(t):=\frac{1}{|D|} \int_{D} a_{i}^{\mu, \xi}(t, x) d x\left(=\check{a}_{i}(t)-2 \mu \xi\right), \quad i=1,2, \cdots, N,
\end{gathered}
$$


and

$$
\check{a}_{0}^{\mu, \xi}(t):=\frac{1}{|D|} \int_{D} a_{0}^{\mu, \xi}(t, x) d x\left(=\check{a}_{0}(t)-\mu \sum_{i=1}^{N} \check{a}_{i}(t) \xi+\mu^{2}\right),
$$

where $|D|$ is the Lebesgue measure of $D$. Let $\check{\lambda}(\mu, \xi ; a)$ be the principal eigenvalue of the following space averaged equation of (2.8),

$$
u_{t}=\Delta u+\sum_{i=1}^{N} \check{a}_{i}^{\mu, \xi}(t) \frac{\partial u}{\partial x_{i}}+\check{a}_{0}^{\mu, \xi}(t) u, \quad x \in \mathbb{R}^{N},
$$

together with the boundary condition (2.2) and $\check{a}=\left(\check{a}_{i}, \check{a}_{0}\right)$.

Let

$$
\begin{gathered}
\bar{a}_{i}:=\frac{\int_{D} \hat{a}_{i}(x) d x}{|D|}, \quad i=1,2, \cdots, N, \\
\bar{a}_{0}:=\frac{\int_{D} \hat{a}_{0}(x) d x}{|D|}, \\
\bar{a}_{i}^{\mu, \xi}:=\frac{\int_{D} \hat{a}_{i}^{\mu, \xi}(x) d x}{|D|}\left(=\bar{a}_{i}-2 \mu \xi_{i}\right), \quad i=1,2, \cdots, N,
\end{gathered}
$$

and

$$
\bar{a}_{0}^{\mu, \xi}:=\frac{\int_{D} \hat{a}_{0}^{\mu, \xi}(x) d x}{|D|}\left(=\bar{a}_{0}-\mu \sum_{i=1}^{N} \bar{a}_{i} \xi+\mu^{2}\right) .
$$

Let $\bar{\lambda}(\mu, \xi ; a)$ be the principal eigenvalue of the following time-space averaged equation of (2.8),

$$
u_{t}=\Delta u+\sum_{i=1}^{N} \bar{a}_{i}^{\mu, \xi} \frac{\partial u}{\partial x_{i}}+\bar{a}_{0}^{\mu, \xi} u, \quad x \in \mathbb{R}^{N},
$$

together with the boundary condition (2.2) and $\bar{a}=\left(\bar{a}_{i}, \bar{a}_{0}\right)$.

Theorem 2.10. (1) If $a_{i}(t, x) \equiv a_{i}(t)$ and $a_{0}(t, x) \equiv a_{0}(t)$, then

$$
\lambda(\mu, \xi ; a)=\hat{\lambda}(\mu, \xi ; a)=\lambda(\mu, \xi ; \hat{a})=\hat{a}_{0}-\mu \sum_{i=1}^{N} \hat{a}_{i} \xi_{i}+\mu^{2}
$$

for any $\mu \in \mathbb{R}$ and $\xi \in S^{N-1}$.

(2) If $a_{i}(t, x) \equiv a_{i}(x)$, then

$$
\lambda(\mu, \xi ; a) \geq \hat{\lambda}(\mu, \xi ; a)
$$

for any $\mu \in \mathbb{R}$ and $\xi \in S^{N-1}$, and $\lambda(\mu, \xi ; a)=\hat{\lambda}(\mu, \xi ; a)$ for some $\mu \in \mathbb{R}$ and $\xi \in S^{N-1}$ if and only if $a_{0}(t, x)$ is of the form $a_{0}(t, x)=a_{01}(t)+a_{02}(x)$.

(3) If $a_{i}(t, x) \equiv a_{i}$ and $a_{0}(t, x) \equiv a_{0}(x)$, then

$$
\lambda(\mu, \xi ; a) \geq \check{\lambda}(\mu, \xi ; a)=\check{a}_{0}^{\mu, \xi}
$$

for any $\mu \in \mathbb{R}$ and $\xi \in S^{N-1}$, and $\lambda(\mu, \xi ; a)=\check{\lambda}(\mu, \xi ; a)$ for some $\mu \in \mathbb{R}$ and $\xi \in S^{N-1}$ if and only if $a_{0}(x) \equiv a_{0}$.

(4) If $a_{i}(t, x) \equiv a_{i}$, then

$$
\lambda(\mu, \xi ; a) \geq \hat{\lambda}(\mu, \xi ; a) \geq \bar{\lambda}(\mu, \xi ; a)=\bar{a}_{0}^{\mu, \xi}
$$

for any $\mu \in \mathbb{R}$ and $\xi \in S^{N-1}$, and $\lambda(\mu, \xi ; a)=\hat{\lambda}(\mu, \xi ; a)=\bar{\lambda}(\mu, \xi ; a)$ for some $\mu \in \mathbb{R}$ and $\xi \in S^{N-1}$ if and only if $a_{0}(t, x) \equiv a_{0}(t)$. 
Proof. (1) If $a_{i}(t, x)=a_{i}(t)$ and $a_{0}(t, x)=a_{0}(t)$, then $\phi\left(a^{\mu, \xi}\right) \equiv 1$ and

$$
\lambda(\mu, \xi ; a)=\lim _{t \rightarrow \infty} \frac{1}{t} \int_{0}^{t}\left(a_{0}(s)-\mu \sum_{i=1}^{N} a_{i}(s) \xi_{i}+\mu^{2}\right) d s
$$

and

$$
\hat{\lambda}(\mu, \xi ; a)=\hat{a}_{0}^{\mu, \xi} .
$$

Therefore $\lambda(\mu, \xi ; a)=\hat{\lambda}(\mu, \xi ; a)=\hat{a}_{0}^{\mu, \xi}$ for any $\mu \in \mathbb{R}$ and $\xi \in S^{N-1}$.

(2) It follows from the arguments in [41.

(3) Note that

$$
\frac{\Delta \phi^{\mu, \xi}(x ; a)}{\phi^{\mu, \xi}(\cdot ; a)}+\frac{\sum_{i=1}^{N} a_{i}^{\mu, \xi} \partial \phi^{\mu, \xi}(x ; a) / \partial x_{i}}{\phi^{\mu, \xi}(x ; a)}+a_{0}^{\mu, \xi}(x)=\lambda(\mu, \xi ; a),
$$

where $\phi^{\mu, \xi}(x ; a)=\phi\left(a^{\mu, \xi}\right)(x)$. This implies that

$$
\begin{aligned}
\lambda(\mu, \xi ; a) & =\check{a}_{0}^{\mu, \xi}+\frac{1}{|D|} \int_{D} \frac{\Delta \phi^{\mu, \xi}(x ; a)}{\phi^{\mu, \xi}(x ; a)} d x \\
& =\check{a}_{0}^{\mu, k}+\frac{1}{|D|} \int_{D} \frac{\sum_{i=1}^{N}\left(\partial \phi^{\mu, \xi}(x ; a) / \partial x_{i}\right)^{2}}{\left(\phi^{\mu, \xi}(x ; a)\right)^{2}} d x \\
& \geq \check{a}_{0}^{\mu, \xi}=\check{\lambda}(\mu, \xi ; a)
\end{aligned}
$$

for any $\mu \in \mathbb{R}$ and $\xi \in S^{N-1}$. Moreover, $\lambda(\mu, \xi ; a)=\check{\lambda}(\mu, \xi ; a)$ for some $\mu \in \mathbb{R}$ and $\xi \in S^{N-1}$ if and only if $a_{0}^{\mu, \xi}(x) \equiv a_{0}^{\mu, \xi}$, which is equivalent to $a_{0}(x) \equiv a_{0}$.

(4) It follows from (2) and (3).

\section{Notation, DEFinitions, AND BASIC PROperties}

In this section, we first introduce standing notation in subsection 3.1. Then in subsection 3.2, we recall the definitions of spreading and generalized propagating speed intervals as well as traveling wave solutions introduced in 21] and [58. We present some fundamental properties proved in 21] in subsection 3.3.

Throughout the rest of this paper, we assume (H1) and (H2). $a_{i}(t, x)(i=$ $1,2, \cdots, N)$ are the functions in (1.1).

3.1. Notation. Consider (1.1). We also consider all the space shifted equations (1.5) of (1.1). To emphasis the dependence of (1.5) on $y$, we may write it as (1.5) $y$.

Let

$$
\begin{aligned}
X: & =C_{u n i f}^{b}\left(\mathbb{R}^{N}, \mathbb{R}\right) \\
& =\left\{u: \mathbb{R}^{N} \rightarrow \mathbb{R} \mid u \text { is uniformly continuous and bounded }\right\},
\end{aligned}
$$

equipped with uniform convergence topology. For given $u_{1}, u_{2} \in X$, we write

$$
u_{1} \leq u_{2} \quad\left(u_{1}<u_{2}\right) \quad \text { if } \quad u_{1}(x) \leq u_{2}(x) \quad\left(u_{1}(x)<u_{2}(x)\right) \quad \text { for } \quad x \in \mathbb{R}^{N} .
$$

It follows from classical theory for parabolic equations (see [16], 20]) that for any $u_{0} \in X$, any $s \in \mathbb{R}$, and any $y \in \mathbb{R}^{N}$, (1.5) has a unique (local) classical solution $u\left(t, \cdot ; s, y, u_{0}\right)$ with $u\left(s, \cdot ; s, y, u_{0}\right)=u_{0}(\cdot)$. We may write $u\left(t, x ; s, 0, u_{0}\right)$ as $u\left(t, x ; s, u_{0}\right)$, which is the solution of (1.1) with $u\left(s, x ; s, u_{0}\right)=u_{0}(x)$.

Let $X_{L}$ be as in (2.3). Note that $X_{L} \subset X$ and if $u_{0} \in X_{L}$, then $u\left(t, \cdot ; s, y, u_{0}\right) \in$ $X_{L}$ for any $y \in \mathbb{R}^{N}$ and $t \geq s$ at which $u\left(t, \cdot ; s, y, u_{0}\right)$ exists. 
For a given globally Hölder continuous function $a_{0}(t, x)$ which is uniformly almost periodic in $t$ and periodic in $x_{j}$ with period $p_{j}(j=1,2, \cdots, N)$, consider the linear equation,

$$
\frac{\partial u}{\partial t}=\Delta u+\sum_{i=1}^{N} a_{i}(t, x) \frac{\partial u}{\partial x_{i}}+a_{0}(t, x) u, \quad x \in \mathbb{R}^{N},
$$

complemented with the periodic boundary condition,

$$
\begin{aligned}
& u\left(t, x_{1}, \cdots, x_{j-1}, x_{j}+p_{j}, x_{j+1}, \cdots, x_{N}\right) \\
& \quad=u\left(t, x_{1}, \cdots, x_{j-1}, x_{j}, x_{j+1}, \cdots, x_{N}\right), \quad j=1,2, \cdots, N .
\end{aligned}
$$

For any $\mu \geq 0$ and $\xi \in S^{N-1}$, also consider

$$
\frac{\partial u}{\partial t}=\Delta u+\sum_{i=1}^{N} a_{i}^{\mu, \xi}(t, x) \frac{\partial u}{\partial x_{i}}+a_{0}^{\mu, \xi}(t, x) u, \quad x \in \mathbb{R}^{N},
$$

complemented with the periodic boundary condition (3.3), where, as in (1.4), $a_{i}^{\mu, \xi}(t, x)=a_{i}(t, x)-2 \mu \xi_{i}(i=1,2, \cdots, N)$ and

$$
a_{0}^{\mu, \xi}(t, x)=a_{0}(t, x)-\mu \sum_{i=1}^{N} a_{i}(t, x) \xi_{i}+\mu^{2} .
$$

Note that by (H2), $u \equiv 0$ is an equilibrium solution of (1.1). If $a_{0}(t, x)=$ $f_{u}(t, x, 0):=\frac{\partial f}{\partial u}(t, x, 0)$, then (3.2) is the linearized equation of (1.1) at $u \equiv 0$.

Throughout this section, $a_{0}(t, x)\left(a_{0}(t)\right)$ denotes a globally Hölder continuous function which is uniformly almost periodic in $t$ and periodic in $x_{j}$ with period $p_{j}$ $(j=1,2, \cdots, N)$ (almost periodic in $t)$, unless specified otherwise. We say that $a_{0}(t, x)$ is of the form $a_{0}(t, x)=a_{01}(t)+a_{02}(x)$ if there are $a_{01}(t)$ which is almost periodic in $t$ and $a_{02}(x)$ which is periodic in $x_{j}$ with period $p_{j}(j=1,2, \cdots, N)$ such that $a_{0}(t, x)=a_{01}(t)+a_{02}(x)$ for $t \in \mathbb{R}$ and $x \in \mathbb{R}^{N}$.

Let $a=\left(\left\{a_{i}\right\}_{i=1}^{N}, a_{0}\right)$ and $\lambda(\mu, \xi ; a)$ be the principal Lyapunov exponent of (3.4) and (3.3) or (1.6). Since $a_{i}(i=1,2, \cdots, N)$ are as in (1.1) and are fixed, we may write $\lambda(\mu, \xi ; a)$ as $\lambda\left(\mu, \xi ; a_{0}\right)$ if no confusion occurs. Note that $\lambda\left(0, \xi ; a_{0}\right)$ is independent of $\xi \in S^{N-1}$, and we may write it as $\lambda\left(a_{0}\right)$. Then $\lambda\left(a_{0}\right)$ is the principal Lyapunov exponent of (3.2) and (3.3). As mentioned in section 2, when $a_{i}(t, x)$ $(i=1,2, \cdots, N)$ and $a_{0}(t, x)$ are periodic in $t$ with the same period $T, \lambda\left(\mu, \xi ; a_{0}\right)=$ $\lambda^{*}\left(\mu, \xi ; a_{0}\right)$, where $\lambda^{*}\left(\mu, \xi ; a_{0}\right)$ is the principal eigenvalue of (1.4).

Let $c_{l}\left(\xi ; a_{0}\right)$ and $c_{l}(\xi)$ be as in (1.7) and (1.8), respectively. Recall that $c_{l}(\xi)$ is called the linear spreading speed for (1.1).

Observe that following from the arguments in [61, there holds

$$
u_{\mathrm{inf}}^{+}:=\inf _{t \in \mathbb{R}, x \in \mathbb{R}^{N}} u^{+}(t, x)>0,
$$

and there is no solution $u(t, x)$ of (1.1) which is almost periodic in $t$, periodic in $x_{j}$ with period $p_{j}(j=1,2, \cdots, N)$, and $0<u(t, x)<u^{+}(t, x)$ for $t \in \mathbb{R}, x \in \mathbb{R}^{N}$. By (H2) and the comparison principle for parabolic equations, for any $u_{0} \in X$ with $u_{0} \geq 0$, any $s \in \mathbb{R}$, and any $y \in \mathbb{R}^{N}, u\left(t+s, \cdot ; s, y, u_{0}\right)$ exists for all $t \geq 0$.

Also observe that examples of functions $f$ satisfying (H1) and (H2) include those which satisfy that $f(t, x, 0)=0, \lambda\left(a_{0}\right)>0\left(a_{0}(t, x)=f_{u}(t, x, 0)\right), f(t, x, u)<0$ for $u \gg 1$, and $\frac{\partial}{\partial u}\left(\frac{f(t, x, u)}{u}\right)<0$ for $u>0$ (see the arguments in [61]). 
Throughout this paper, $D$ and $S^{N-1}$ denote the subsets of $\mathbb{R}^{N}$ defined as follows:

$$
D=\left[0, p_{1}\right] \times\left[0, p_{2}\right] \times \cdots \times\left[0, p_{N}\right]
$$

and

$$
S^{N-1}=\left\{\xi \in \mathbb{R}^{N} \mid\|\xi\|=1\right\}
$$

where $\|\cdot\|$ is the usual norm in $\mathbb{R}^{N}$. If (1.1) is periodic in $t$, as mentioned in the Introduction, for any $\xi \in S^{N-1}$, there is a so-called spreading speed of (1.1) in the direction of $\xi$. We denote it by $c^{*}(\xi)$.

For given $u_{0} \in X$ and $\xi \in S^{N-1}$, we define

$$
\liminf _{x \cdot \xi \rightarrow-\infty} u_{0}(x)=\lim _{r \rightarrow-\infty} \inf _{x \in \mathbb{R}^{N}, x \cdot \xi \leq r} u_{0}(x)
$$

and

$$
\limsup _{x \cdot \xi \rightarrow \infty} u_{0}(x)=\lim _{r \rightarrow \infty} \sup _{x \in \mathbb{R}^{N}, x \cdot \xi \geq r} u_{0}(x) .
$$

For given $u(t, \cdot) \in X, \xi \in S^{N-1}$, and $c \in \mathbb{R}$, we define

$$
\begin{aligned}
& \liminf _{x \cdot \xi \leq c t, t \rightarrow \infty} u(t, x)=\liminf _{t \rightarrow \infty} \inf _{x \in \mathbb{R}^{N}, x \cdot \xi \leq c t} u(t, x), \\
& \limsup _{x \cdot \xi \geq c t, t \rightarrow \infty} u(t, x)=\limsup _{t \rightarrow \infty} \sup _{x \in \mathbb{R}^{N}, x \cdot \xi \geq c t} u(t, x),
\end{aligned}
$$

and

$$
\begin{aligned}
& \limsup _{\|x\| \geq c t, t \rightarrow \infty} u(t, x)=\limsup _{t \rightarrow \infty} \sup _{x \in \mathbb{R}^{N},\|x\| \geq c t} u(t, x), \\
& \liminf _{|x \cdot \xi| \leq c t, t \rightarrow \infty} u(t, x)=\liminf _{t \rightarrow \infty} \inf _{x \in \mathbb{R}^{N},|x \cdot \xi| \leq c t} u(t, x) .
\end{aligned}
$$

For given $u(t, \cdot ; z) \in X$ ( $z$ belongs to some parameter set $Z), \xi \in S^{N-1}$, and $c \in \mathbb{R}$, we say that $\liminf x \cdot \xi \leq c t, t \rightarrow \infty u(t, x ; z) \geq u^{*}$ uniformly in $z \in Z$ if for any $\epsilon>0$, there is $T>0$ such that

$$
u(t, x ; z) \geq u^{*}-\epsilon \quad \text { for } \quad t \geq T, x \in \mathbb{R}^{N} \text { with } \quad x \cdot \xi \leq c t, z \in Z .
$$

We define $\lim \sup _{x \cdot \xi \geq c t, t \rightarrow \infty} u(t, x ; z) \leq u^{*}, \lim _{\sup _{\|x\| \geq c t, t \rightarrow \infty} u(t, x ; z) \leq u^{*} \text {, and }}$ $\liminf _{|x \cdot \xi| \leq c t, t \rightarrow \infty} u(t, x ; z) \geq u^{*}$ uniformly in $z \in Z$ in a similar manner.

For a given function $g(t, x)(g(t, x, u))$, we write $g(t, x) \equiv g(t)$ or $g(t, x) \equiv g(x)$ or $g(t, x) \equiv g(g(t, x, u) \equiv g(t, u)$ or $g(t, x) \equiv g(x, u)$ or $g(t, x, u) \equiv g(u))$ if $g(t, x)$ $(g(t, x, u))$ is independent of $x$ or $t$ or both $t$ and $x$.

For a given globally Hölder continuous function $g(t, x)$ which is uniformly almost periodic in $t$ and periodic in $x_{j}$ with period $p_{j}(j=1,2, \cdots, N)$, we say that it can be approximated by time periodic functions if there are globally Hölder continuous functions $g_{n}(t, x)$ which are periodic in $t$ with period $T_{n}$ and periodic in $x_{j}$ with period $p_{j}(j=1,2, \cdots, N)$ such that $g_{n}(t, x) \rightarrow g(t, x)$ uniformly in $t \in \mathbb{R}$ and $x \in \mathbb{R}^{N}$.

3.2. Definitions. In this subsection, we recall the definitions of spreading speed intervals, generalized propagating speed intervals, and traveling wave solutions.

For a given $\xi \in S^{N-1}$, let

$$
\begin{aligned}
X_{1}^{+}(\xi)=\left\{u_{0} \in X \mid 0\right. & \leq u_{0} \leq \sup _{x \in \mathbb{R}^{\mathbb{N}}} u_{0}(x)<u_{\text {inf }}^{+}, \\
0 & \left.<\liminf _{x \cdot \xi \rightarrow-\infty} u_{0}(x)<u_{\text {inf }}^{+}, u_{0}(x)=0 \text { for } \quad x \cdot \xi \gg 1\right\}
\end{aligned}
$$


and

$$
X_{2}^{+}(\xi)=\left\{u_{0} \in X \mid u_{0} \geq 0, \liminf _{x \cdot \xi \rightarrow-\infty} u_{0}(x)>0, \limsup _{x \cdot \xi \rightarrow \infty} u_{0}(x)=0\right\} .
$$

Recall that $u_{0} \in X_{2}^{+}(\xi)$ is called a front in the direction of $\xi$ and that $u\left(t, x ; s, y, u_{0}\right)$ with $u_{0} \in X_{2}^{+}(\xi)$ is called a front solution in the direction of $\xi$.

Definition 3.1. The solution $u \equiv 0$ of (1.1) is called linearly unstable with respect to space periodic perturbations if $\lambda\left(a_{0}\right)>0$, where $a_{0}(t, x)=f_{u}(t, x, 0)$. The solution $u=u^{+}(t, x)$ of (1.1) is called globally stable with respect to space periodic perturbations if for any $u_{0} \in X_{L}, u_{0}(x) \geq 0$ and $u_{0}(x) \not \equiv 0$,

$$
\left\|u\left(t+s, \cdot ; s, y, u_{0}(\cdot)\right)-u^{+}(t+s, \cdot+y)\right\|_{X_{L}} \rightarrow 0 \quad \text { as } \quad t \rightarrow \infty
$$

uniformly in $s \in \mathbb{R}$ and $y \in \mathbb{R}^{N}$.

Definition 3.2 (Spreading speed interval, 21]). For a given vector $\xi \in S^{N-1}$, let

$$
\begin{aligned}
C_{\text {inf }}^{*}(\xi)=\{c \mid & \forall u_{0} \in X_{1}^{+}(\xi), \\
& \left.\liminf _{x \cdot \xi \leq c t, t \rightarrow \infty}\left(u\left(t+s, x ; s, u_{0}\right)-u^{+}(t+s, x)\right)=0 \text { uniformly in } s \in \mathbb{R}\right\}
\end{aligned}
$$

and

$C_{\text {sup }}^{*}(\xi)=\left\{c \mid \forall u_{0} \in X_{1}^{+}(\xi), \limsup _{x \cdot \xi \geq c t, t \rightarrow \infty} u\left(t+s, x ; s, u_{0}\right)=0\right.$ uniformly in $\left.s \in \mathbb{R}\right\}$.

Define

$$
c_{\mathrm{inf}}^{*}(\xi)=\sup \left\{c \mid c \in C_{\mathrm{inf}}^{*}(\xi)\right\}, \quad c_{\mathrm{sup}}^{*}(\xi)=\inf \left\{c \mid c \in C_{\mathrm{sup}}^{*}(\xi)\right\} .
$$

The interval $\left[c_{\mathrm{inf}}^{*}(\xi), c_{\mathrm{sup}}^{*}(\xi)\right]$ is called the spreading speed interval of (1.1) in the direction of $\xi$.

Definition 3.3 (Generalized propagating speed interval, [21]). For a given $\xi \in$ $S^{N-1}$ and a given $u_{0} \in X_{2}^{+}(\xi)$, let

$$
\begin{aligned}
C_{\text {inf }}\left(u_{0}, \xi\right)= & \left\{c \mid \liminf _{x \cdot \xi \leq c t, t \rightarrow \infty}\left(u\left(t+s, x ; s, y, u_{0}\right)-u^{+}(t+s, x+y)\right)=0\right. \\
& \text { uniformly in } \left.s \in \mathbb{R}, y \in \mathbb{R}^{N}\right\}
\end{aligned}
$$

and

$$
C_{\text {sup }}\left(u_{0}, \xi\right)=\left\{c \mid \limsup _{x \cdot \xi \geq c t, t \rightarrow \infty} u\left(t+s, x ; s, y, u_{0}\right)=0 \quad \text { uniformly in } s \in \mathbb{R}, y \in \mathbb{R}^{N}\right\} .
$$

Let

$$
c_{\text {inf }}\left(u_{0}, \xi\right)=\sup \left\{c \mid c \in C_{\inf }\left(u_{0}, \xi\right)\right\}, \quad c_{\text {sup }}\left(u_{0}, \xi\right)=\inf \left\{c \mid c \in C_{\text {sup }}\left(u_{0}, \xi\right)\right\} .
$$

The interval $\left[c_{\mathrm{inf}}\left(u_{0}, \xi\right), c_{\mathrm{sup}}\left(u_{0}, \xi\right)\right]$ is called the generalized propagating speed interval of $u_{0}$ in the direction of $\xi$.

If $c_{\text {inf }}\left(u_{0}, \xi\right)=c_{\text {sup }}\left(u_{0}, \xi\right)$, then $u\left(t, \cdot ; s, u_{0}\right)$ is called a front solution of (1.1) in the direction of $\xi$ with propagation speed $c\left(u_{0}, \xi\right):=c_{\mathrm{inf}}\left(u_{0}, \xi\right)\left(=c_{\mathrm{sup}}\left(u_{0}, \xi\right)\right)$.

Definition 3.4 (Traveling wave, 21], [58]). $u(t, x)$ is said to be a traveling wave solution of (1.1) connecting $u^{+}$and 0 in the direction of $\xi \in S^{N-1}$ if there exist $U(x ; s, y, \xi)$ and $c(t ; s, y, \xi)$ satisfying the following properties:

(1) $U(\cdot ; s, y, \xi) \in X$ is continuous in $s \in \mathbb{R}$ and $y \in \mathbb{R}^{N}$. Moreover, $U(\cdot ; s, y, \xi)$ is recurrent in $s$ (see Definition A.3 in Appendix A for a definition) and periodic in $y_{j}$ with period $p_{j}(j=1,2, \cdots, N)$. 
(2)

$$
\lim _{\xi \cdot x \rightarrow-\infty}\left(U(x ; s, y, \xi)-u^{+}(s, x+y)\right)=0, \quad \lim _{\xi \cdot x \rightarrow \infty} U(x ; s, y, \xi)=0
$$

uniformly in $s \in \mathbb{R}$ and $y \in \mathbb{R}^{N}$.

(3) $u(0, x)=U(x ; 0,0, \xi)$ and

$$
u(t+s, x ; s, y, U(\cdot ; s, y, \xi))=U(x-c(t ; s, y, \xi) \xi ; t+s, y+c(t ; s, y, \xi), \xi)
$$

for any $s \in \mathbb{R}, y \in \mathbb{R}^{N}$.

We say $\{U(\cdot ; s, y, \xi)\}_{s \in \mathbb{R}, y \in \mathbb{R}^{N}}$ generates a traveling wave solution in the direction of $\xi \in S^{N-1}$ if there is $c(t ; s, y, \xi)$ such that $U(x ; s, y, \xi)$ and $c(t ; s, y, \xi)$ satisfy the above properties. In this case, we define

$$
c_{\mathrm{inf}}(U, \xi)=\liminf _{t \rightarrow \infty} \inf _{s \in \mathbb{R}, y \in \mathbb{R}^{N}} \frac{c(t ; s, y, \xi)}{t}
$$

and

$$
c_{\text {sup }}(U, \xi)=\limsup _{t \rightarrow \infty} \sup _{s \in \mathbb{R}, y \in \mathbb{R}^{N}} \frac{c(t ; s, y, \xi)}{t}
$$

and call $\left[c_{\text {inf }}(U, \xi), c_{\text {sup }}(U, \xi)\right]$ the averaged speed interval of the traveling wave so-

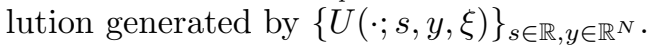

3.3. Fundamental properties. In this subsection, we present some fundamental properties for spreading and generalized propagating speed intervals proved in [21] for use in later sections and/or for the reader's reference.

Proposition 3.5 (21], Theorem 2.1).

(1) (Boundedness) For any $\xi \in S^{N-1},\left[c_{\mathrm{inf}}^{*}(\xi), c_{\mathrm{sup}}^{*}(\xi)\right]$ is a finite interval.

(2) (Recovery of the classical spreading speed) If (1.1) is periodic in then $c_{\text {inf }}^{*}(\xi)=c_{\text {sup }}^{*}(\xi)=c^{*}(\xi)$ for any $\xi \in S^{N-1}$.

(3) (Minimality)

(i) For any $\xi \in S^{N-1}$ and $u_{0} \in X_{2}^{+}(\xi), c_{\mathrm{inf}}^{*}(\xi) \leq c_{\mathrm{inf}}\left(u_{0}, \xi\right)$ and $c_{\mathrm{sup}}^{*}(\xi) \leq$ $c_{\text {sup }}\left(u_{0}, \xi\right)$.

(ii) For any $\xi \in S^{N-1}$ and $u_{0} \in X_{1}^{+}(\xi), c_{\mathrm{inf}}^{*}(\xi)=c_{\mathrm{inf}}\left(u_{0}, \xi\right)$ and $c_{\mathrm{sup}}^{*}(\xi)=$ $c_{\text {sup }}\left(u_{0}, \xi\right)$.

(4) (Minimality) If $\{U(\cdot ; s, y, \xi)\}_{s \in \mathbb{R}, y \in \mathbb{R}^{N}}$ generates a traveling wave solution of (1.1) connecting $u^{+}$and $u^{-} \equiv 0$ in the direction of $\xi \in S^{N-1}$, then $c_{\text {sup }}^{*}(\xi) \leq c_{\text {sup }}(U, \xi)$ and $c_{\mathrm{inf}}^{*}(\xi) \leq c_{\mathrm{inf}}(U, \xi)$.

Proposition 3.6 ([21], Theorem 2.2).

(1) For any $0<\delta<u_{\text {inf }}^{+}, \xi \in S^{N-1}, u_{0} \in X_{1}^{+}(\xi), c^{\prime}<c_{\mathrm{inf}}^{*}(\xi)$ and $c^{\prime \prime}>c_{\mathrm{sup}}^{*}(\xi)$,

$$
\liminf _{x \cdot \xi \leq c^{\prime} t, t \rightarrow \infty}\left(u\left(t+s, x ; s, y, u_{0}\right)-u^{+}(t+s, x+y)\right)=0,
$$

and

$$
\limsup _{x \cdot \xi \geq c^{\prime \prime} t, t \rightarrow \infty} u\left(t+s, x ; s, y, u_{0}\right)=0
$$

uniformly in $s \in \mathbb{R}$ and $y \in \mathbb{R}^{N}$. 
(2) Assume that $0 \leq u_{0}<u_{\mathrm{inf}}^{+}\left(u_{0} \in X\right)$ and $u_{0}(x)=0$ for $\|x\| \gg 1$. Then for any $c>\sup _{\|\xi\|=1} c_{\text {sup }}^{*}(\xi)$,

$$
\limsup _{\|x\| \geq c t, t \rightarrow \infty} u\left(t+s, x ; s, y, u_{0}\right)=0
$$

uniformly in $s \in \mathbb{R}$ and $y \in \mathbb{R}^{N}$.

(3) Assume that $\xi \in S^{N-1}$ and $0<c<\min \left\{c_{\mathrm{inf}}^{*}(\xi), c_{\mathrm{inf}}^{*}(-\xi)\right\}$. Then for any $\sigma>0$, there is $r_{\sigma}>0$ such that for any $u_{0} \geq 0\left(u_{0} \in X\right)$ with $u_{0}(x) \geq \sigma$ for $|x \cdot \xi| \leq r_{\sigma}$,

$$
\liminf _{|x \cdot \xi| \leq c t, t \rightarrow \infty}\left(u\left(t+s, x ; s, y, u_{0}\right)-u^{+}(t+s, x+y)\right)=0
$$

uniformly in $s \in \mathbb{R}$ and $y \in \mathbb{R}^{N}$.

We remark that Propositions 3.5 and 3.6 justify the nature of the notion of spreading speed intervals and show that the concept of spreading speed intervals is the natural extension of the spreading speeds for time independent or periodic KPP models.

Proposition 3.7. (1) ([21, Lemma 3.2]) Let $\xi \in S^{N-1}, u_{0} \in X_{2}^{+}(\xi)$, and $c \in \mathbb{R}$ be given. If there are $\delta_{0}$ and $T_{0}>0$ such that

$$
\liminf _{x \cdot \xi \leq c n T_{0}, n \rightarrow \infty} u\left(n T_{0}+s, x ; s, y, u_{0}\right) \geq \delta_{0}
$$

uniformly in $s \in \mathbb{R}$ and $y \in \mathbb{R}^{N}$, then for any $c^{\prime}<c$,

$$
\liminf _{x \cdot \xi \leq c^{\prime} t, t \rightarrow \infty}\left(u\left(t+s, x ; s, y, u_{0}\right)-u^{+}(t+s, x+y)\right)=0
$$

uniformly in $s \in \mathbb{R}$ and $y \in \mathbb{R}^{N}$.

(2) (21, Lemma 3.4]) Assume that there is $u_{0}^{*} \in X_{1}^{+}(\xi)$ such that

$$
\liminf _{x \cdot \xi \leq c t, t \rightarrow \infty}\left(u\left(t+s, x ; s, y, u_{0}^{*}\right)-u^{+}(t+s, x+y)\right)=0
$$

uniformly in $s \in \mathbb{R}$ and $y \in \mathbb{R}^{N}$. Then $c \leq c_{\text {inf }}^{*}(\xi)$.

\section{Spreading SPEeds And generalized PROpagating SPEeds IN THE PARTIALLY SPATIALLY HOMOGENEOUS CASE}

In this section, we study spatial spread and front propagation dynamics in the partially spatially homogeneous case.

Throughout this section, we assume that $a_{i}(t, x) \equiv a_{i}(t), i=1,2, \cdots, N$, and that $a_{0}(t)$ is some given globally Hölder continuous almost periodic function unless specified otherwise. For given $a_{0}(t), \mu \in \mathbb{R}$, and $\xi \in S^{N-1}, \lambda\left(\mu, \xi ; a_{0}\right)$ is the principal Lyapunov exponent of (3.4) and (3.3), and $c_{l}\left(\xi ; a_{0}\right)$ and $c_{l}(\xi)$ are as in (1.7) and (1.8), respectively. If $\lambda\left(a_{0}\right)>0, \mu^{*}\left(a_{0}\right)>0$ is such that $c_{l}\left(\xi ; a_{0}\right)=\frac{\lambda\left(\mu^{*}\left(a_{0}\right), \xi ; a_{0}\right)}{\mu^{*}\left(a_{0}\right)}$ (it will be seen in the following that such $\mu^{*}\left(a_{0}\right)$ exists and is unique).

The following two theorems are the main results of this section.

Theorem 4.1 (Spatial spread).

(1) (Upper bound) Assume that $f(t, x, u) \leq a_{0}(t) u$ for $t \in \mathbb{R}, x \in \mathbb{R}^{N}$, and $u \geq 0$. Then

$$
c_{\mathrm{sup}}^{*}(\xi) \leq c_{l}\left(\xi ; a_{0}\right) \quad \text { for any } \quad \xi \in S^{N-1} .
$$


(2) (Lower bound) Assume that $\lambda\left(a_{0}\right)>0$ and $f(t, x, u) \geq a_{0}(t) u$ for $t \in \mathbb{R}$, $x \in \mathbb{R}^{N}$, and $0 \leq u \ll 1$. Then

$$
c_{\mathrm{inf}}^{*}(\xi) \geq c_{l}\left(\xi ; a_{0}\right) \quad \text { for any } \quad \xi \in S^{N-1} .
$$

In particular, if $f_{u}(t, x, 0) \equiv f_{u}(t, 0,0)$, then

$$
c_{\mathrm{inf}}^{*}(\xi) \geq c_{l}(\xi) \quad \text { for any } \xi \in S^{N-1} .
$$

(3) (Variational principle/linear determinacy) If $f(t, x, u) \leq f_{u}(t, x, 0) u$ for $t \in \mathbb{R}, x \in \mathbb{R}^{N}$, and $u \geq 0$, and $f_{u}(t, x, 0) \equiv f_{u}(t, 0,0)$, then

$$
c_{\mathrm{inf}}^{*}(\xi)=c_{\mathrm{sup}}^{*}(\xi)=c_{l}(\xi) \quad \text { for any } \xi \in S^{N-1} .
$$

Recall that $c_{l}(\xi)$ is the linear spreading speed of (1.1) in the direction of $\xi$. If $c_{\mathrm{inf}}^{*}(\xi)=c_{\mathrm{sup}}^{*}(\xi)=c_{l}(\xi)$, as in the periodic case, we say (1.1) satisfies the linear determinacy (see 32, 33, 67, etc. for the study on linear determinacy) and the characterization $c_{\text {inf }}^{*}(\xi)=c_{\text {sup }}^{*}(\xi)=c_{l}(\xi)=\inf _{\mu>0} \frac{\lambda\left(\mu, \xi ; a_{0}\right)}{\mu}\left(a_{0}(t)=f_{u}(t, 0,0)\right)$ is called the variational principle for $c^{*}(\xi):=c_{\mathrm{inf}}^{*}(\xi)\left(=c_{\mathrm{sup}}^{*}(\xi)\right)$.

Observe that $f(t, x, u) \equiv f(t, u)$ and $f(t, x, u) \equiv u\left(a_{0}(t)-b(t, x) u\right)$ are among the examples which satisfy $f_{u}(t, x, 0) \equiv f_{u}(t, 0,0)$.

Theorem 4.2 (Generalized propagation).

(1) (Upper bound) Assume that $f(t, x, u) \leq a_{0}(t) u$ for $t \in \mathbb{R}, x \in \mathbb{R}^{N}$, and $u \geq 0$. If $\xi \in S^{N-1}$ and $u_{0} \in X_{2}^{+}(\xi)$ satisfy the fact that $u_{0}(x) \leq C e^{-\mu x \cdot \xi}$ for $x \cdot \xi \gg 1$ and some $C>0$ and $\mu>0$, then

$$
c_{\mathrm{sup}}\left(u_{0}, \xi\right) \leq \frac{\lambda\left(\mu, \xi ; a_{0}\right)}{\mu} .
$$

(2) (Lower bound) Assume that $\lambda\left(a_{0}\right)>0$ and $f(t, x, u) \geq a_{0}(t) u$ for $t \in \mathbb{R}$, $x \in \mathbb{R}^{N}$, and $0 \leq u \ll 1$. If $\xi \in S^{N-1}$ and $u_{0} \in X_{2}^{+}(\xi)$ satisfy the fact that $u_{0}(x) \geq C e^{-\mu x \cdot \bar{\xi}}$ for $x \cdot \xi \gg 1$ and some $C>0$ and $0<\mu<\mu^{*}\left(a_{0}\right)$, then

$$
c_{\text {inf }}\left(u_{0}, \xi\right) \geq \frac{\lambda\left(\mu, \xi ; a_{0}\right)}{\mu} .
$$

(3) (Front solutions of constant speeds) Assume that $f(t, x, u) \leq f_{u}(t, x, 0) u$ for $t \in \mathbb{R}, x \in \mathbb{R}^{N}$, and $u \geq 0$, and $f_{u}(t, x, 0) \equiv f_{u}(t, 0,0)$. If $\xi \in S^{N-1}$ and $u_{0} \in X_{2}^{+}(\xi)$ satisfy the fact that $C_{1} e^{-\mu x \cdot \xi} \leq u_{0}(x) \leq C_{2} e^{-\mu x \cdot \xi}$ for $x \cdot \xi \gg 1$ and some $C_{1}, C_{2}>0$ and $0<\mu<\mu^{*}\left(a_{0}\right)$ with $a_{0}(t) \equiv f_{u}(t, 0,0)$, then

$$
c_{\text {inf }}\left(u_{0}, \xi\right)=u_{\text {sup }}\left(u_{0}, \xi\right)=\frac{\lambda\left(\mu, \xi ; a_{0}\right)}{\mu} .
$$

Hence for any $c \geq c_{l}(\xi)$ there is a front solution of (1.1) in the direction of $\xi$ with speed $c$, and there is no front solution in the direction of $\xi$ with speed smaller than $c_{l}(\xi)$.

To prove Theorems 4.1 and 4.2 , we first prove some lemmas.

First of all, without loss of generality, we assume that $a_{i}(t) \equiv 0$. Otherwise, let

$$
\begin{aligned}
v(t, x) & =v\left(t, x_{1}, \cdots, x_{N}\right) \\
& =u\left(t, x_{1}-\int_{0}^{t} a_{1}(s) d s, x_{2}-\int_{0}^{t} a_{2}(s) d s, \cdots, x_{N}-\int_{0}^{t} a_{N}(s) d s\right) .
\end{aligned}
$$


Then $v$ satisfies

$$
v_{t}=\Delta v+\tilde{f}(t, x, v), \quad x \in \mathbb{R}^{N},
$$

where

$$
\begin{aligned}
& \tilde{f}\left(t, x_{1}, x_{2}, \cdots, x_{N}, v\right) \\
& \quad=f\left(t, x_{1}-\int_{0}^{t} a_{1}(s) d s, x_{2}-\int_{0}^{t} a_{2}(s) d s, \cdots, x_{N}-\int_{0}^{t} a_{N}(s) d s, v\right) .
\end{aligned}
$$

It is not difficult to verify that (4.1) satisfies (H1) and (H2). Let

$$
\hat{a}_{i}=\lim _{t \rightarrow \infty} \frac{1}{t} \int_{0}^{t} a_{i}(s) d s \quad(i=1,2, \cdots, N) .
$$

For a given $\xi=\left(\xi_{1}, \xi_{2}, \cdots, \xi_{N}\right)^{\top} \in S^{N-1}$, denote $\left[\tilde{c}_{\text {inf }}^{*}(\xi), \tilde{c}_{\text {sup }}^{*}(\xi)\right]$ as the spreading speed interval of (4.1) in the direction of $\xi \in S^{N-1}$. We have

$$
c_{\mathrm{inf}}^{*}(\xi)=\tilde{c}_{\mathrm{inf}}^{*}(\xi)-\sum_{i=1}^{N} \hat{a}_{i} \xi_{i}, \quad c_{\mathrm{sup}}^{*}(\xi)=\tilde{c}_{\mathrm{sup}}^{*}(\xi)-\sum_{i=1}^{N} \hat{a}_{i} \xi_{i} .
$$

For given $a_{0}(\cdot), \xi=\left(\xi_{1}, \xi_{2}, \cdots, \xi_{N}\right)^{\top} \in S^{N-1}$, and $\mu \in \mathbb{R}$, by Theorem 2.10 (1), we have

$$
\frac{\lambda\left(\mu, \xi ;\left(\left\{a_{i}\right\}_{i=1}^{N}, a_{0}\right)\right)}{\mu}=\frac{\lambda\left(\mu, \xi ;\left(\{0\}_{i=1}^{N}, a_{0}\right)\right)}{\mu}-\sum_{i=1}^{N} \hat{a}_{i} \xi_{i} .
$$

Therefore, in the rest of this section, we assume that $a_{i}(t) \equiv 0$. Hence (1.1) reads as

$$
\frac{\partial u}{\partial t}=\Delta u+f(t, x, u), \quad x \in \mathbb{R}^{N} .
$$

Recall that $u\left(t, \cdot ; s, u_{0}\right)$ denotes the solution of (4.2) with $u\left(s, \cdot ; s, u_{0}\right)=u_{0}(\cdot)$.

For given $a_{0}(t), \mu \in \mathbb{R}$, and $\xi \in S^{N-1}$, (3.4) becomes

$$
\frac{\partial u}{\partial t}=\Delta u+\sum_{i=1}^{N} a_{i}^{\mu, \xi} \frac{\partial u}{\partial x_{i}}+a_{0}^{\mu, \xi}(t) u, \quad x \in \mathbb{R}^{N},
$$

where $a^{\mu, \xi}=\left(\left\{a_{i}^{\mu, \xi}\right\}_{i=1}^{N}, a_{0}^{\mu, \xi}\right), a_{i}^{\mu, \xi}=-2 \mu \xi_{i}(i=1,2, \cdots, N)$, and $a_{0}^{\mu, \xi}(t)=$ $a_{0}(t)+\mu^{2}$. Let $u\left(t, \cdot ; s, u_{0}, a^{\mu, \xi}\right)$ be the solution of (4.3) and (3.3) with $u\left(s, \cdot ; s, u_{0}, a^{\mu, \xi}\right)=u_{0}(\cdot)$ for $u_{0} \in X_{L}$.

Note that for any $\xi \in S^{N-1}$ and $\mu>0$,

$$
\lambda\left(\mu, \xi ; a_{0}\right)=\hat{a}_{0}+\mu^{2},
$$

where $\hat{a}_{0}=\lim _{t-s \rightarrow \infty} \frac{1}{t-s} \int_{s}^{t} a_{0}(\tau) d \tau$ (see Theorem 2.10 (1)). Hence $\lambda\left(\mu, \xi ; a_{0}\right)$ is independent of $\xi$, and we may write it as $\lambda\left(\mu ; a_{0}\right)$. For any $\mu \neq 0$,

$$
\frac{\lambda\left(\mu ; a_{0}\right)}{\mu}=\frac{\hat{a}_{0}}{\mu}+\mu
$$

Therefore if $\lambda\left(a_{0}\right)>0$, there is a unique $\mu^{*}:=\mu^{*}\left(a_{0}\right)>0$ such that

$$
\frac{\lambda\left(\mu^{*} ; a_{0}\right)}{\mu^{*}}=\inf _{\mu>0} \frac{\lambda\left(\mu ; a_{0}\right)}{\mu} .
$$

In the following, we assume that $\lambda\left(a_{0}\right)>0$ unless specified otherwise. Let $0<\mu_{-}^{*}<\mu^{*}<\mu_{+}^{*}$ be fixed, where $\mu^{*}$ is such that (4.4) holds. 
For any $s \in \mathbb{R}, \mu>0, \xi \in S^{N-1}$, and $T>0$, let

$$
\Phi\left(T ; s, a_{0}, \mu, \xi\right) u_{0}=u\left(s+T, \cdot ; s, u_{0}, a^{\mu, \xi}\right)
$$

for $u_{0} \in X_{L}$. Then $\Phi\left(T ; s, a_{0}, \mu, \xi\right): X_{L} \rightarrow X_{L}$ is a compact and strongly monotone operator (i.e., for any bounded subset $E \subset X_{L}, \Phi\left(T ; s, a_{0}, \mu, \xi\right) E$ is relatively compact in $X_{L}$, and for any $u_{0} \in X_{L}$ with $u_{0} \geq 0$ and $u_{0} \neq 0,\left(\Phi\left(T ; s, a_{0}, \mu, \xi\right) u_{0}\right)(x)>$ 0 for $x \in D)$. By the Krein-Rutman theorem, the eigenvalue of $\Phi\left(T ; s, a_{0}, \mu, \xi\right)$ with the largest module is real and simple. We call it the principal eigenvalue of $\Phi\left(T ; s, a_{0}, \mu, \xi\right)$ and denote it by $\lambda_{T}\left(s, a_{0}, \mu, \xi\right)$. Let $e\left(\Phi\left(T ; s, a_{0}, \mu, \xi\right)\right)$ be a corresponding eigenfunction. We have that $e\left(\Phi\left(T ; s, a_{0}, \mu, \xi\right)\right)=$ constant and

$$
\begin{gathered}
\lambda_{T}\left(s, a_{0}, \mu, \xi\right)=e^{\int_{0}^{T}\left(a_{0}(s+\tau)+\mu^{2}\right) d \tau}, \\
\frac{1}{T} \ln \lambda_{T}\left(s, a_{0}, \mu, \xi\right)=\frac{1}{T} \int_{0}^{T}\left(a_{0}(s+\tau)+\mu^{2}\right) d \tau .
\end{gathered}
$$

Note that $\lambda_{T}\left(s, a_{0}, \mu, \xi\right)$ is independent of $\xi$, and we may write it as $\lambda_{T}\left(s, a_{0}, \mu\right)$. It is clear that for $T \gg 1, \frac{1}{T} \int_{0}^{T} a_{0}(s+\tau) d \tau>0$ for any $s \in \mathbb{R}$ (see Theorem A.1), and then there is a unique $\mu^{*}\left(T, s, a_{0}\right)>0$ such that

$$
\frac{1}{T} \frac{\ln \lambda_{T}\left(s, a_{0}, \mu^{*}\left(T, s, a_{0}\right)\right)}{\mu^{*}\left(T, s, a_{0}\right)}=\inf _{\mu>0} \frac{1}{T} \frac{\ln \lambda_{T}\left(s, a_{0}, \mu\right)}{\mu} .
$$

Lemma 4.3 below follows from Theorem A.1.

Lemma 4.3. For any $0<\epsilon<\min \left\{\mu_{+}^{*}-\mu^{*}, \mu^{*}-\mu_{-}^{*}\right\}$, there is $T \geq 1$ such that

$$
\begin{aligned}
\mu^{*}\left(T, s, a_{0}\right) & \in\left(\mu^{*}-\epsilon / 2, \mu^{*}+\epsilon / 2\right), \\
\frac{1}{T} \frac{\ln \lambda_{T}\left(s, a_{0}, \mu^{*}\left(T, s, a_{0}\right)\right)}{\mu^{*}\left(T, s, a_{0}\right)} & \in\left(\frac{\lambda\left(\mu^{*} ; a_{0}\right)}{\mu^{*}}-\epsilon / 2, \frac{\lambda\left(\mu^{*} ; a_{0}\right)}{\mu^{*}}+\epsilon / 2\right)
\end{aligned}
$$

for all $s \in \mathbb{R}$, and there is $\mu_{T} \in\left(\mu^{*}-\epsilon / 2, \mu^{*}+\epsilon / 2\right)$ such that

$$
\mu_{T}<\mu^{*}\left(T, s, a_{0}\right) \quad \text { for all } s \in \mathbb{R} \text {. }
$$

Observe that when $\mu=0$, (4.3) reads as

$$
\frac{\partial u}{\partial t}=\Delta u+a_{0}(t) u
$$

For any $u_{0} \in C\left(\mathbb{R}^{N}, \mathbb{R}\right)$ with $u_{0}(x) \leq M \exp (\alpha\|x\|)$ for $\|x\| \gg 1$ and some $M, \alpha>0$, (4.6) has also a unique solution $u\left(t, \cdot ; s, u_{0}, a_{0}\right)$ with $u\left(s, \cdot ; s, u_{0}, a_{0}\right)=u_{0}(\cdot)$ (see [16]). Put $\tilde{\Phi}\left(t ; s, a_{0}\right) u_{0}:=u\left(t+s, \cdot ; s, u_{0}, a_{0}\right)$ for such initial $u_{0}$. Then for any $\mu \in \mathbb{R}, \xi \in S^{N-1}$, and $u_{0} \in X_{L}$, there holds

$$
\left(\Phi\left(T ; s, a_{0}, \mu, \xi\right) u_{0}\right)(x)=e^{\mu x \cdot \xi}\left(\tilde{\Phi}\left(T ; s, a_{0}\right) u_{0}^{\mu, \xi}\right)(x),
$$

where $u_{0}^{\mu, \xi}(x)=e^{-\mu x \cdot \xi} u_{0}(x)$ and $u_{0} \in X_{L}$.

Observe also that there is a nonnegative measure $m(y, d y)$ independent of $\mu, \xi$ and $a_{0}$ such that

$$
\left(\tilde{\Phi}\left(T ; s, a_{0}\right) u_{0}^{\mu, \xi}\right)(x)=e^{\int_{0}^{T} a_{0}(s+\tau) d \tau} \cdot \int u_{0}^{\mu, \xi}(x-y) m(y, d y)
$$

for all $s \in \mathbb{R}$. Hence

$$
\Phi\left(T ; s, a_{0}, \mu, \xi\right) u_{0}(x)=e^{\int_{0}^{T} a_{0}(s+\tau) d \tau} \cdot \int e^{\mu y \cdot \xi} u_{0}(x-y) m(y, d y)
$$


and

$$
\frac{1}{T} \ln \lambda_{T}\left(s, a_{0}, \mu\right)=\frac{1}{T}\left(\int_{0}^{T} a_{0}(s+\tau)+\ln \int e^{\mu y \cdot \xi} m(y, d y)\right) .
$$

Let $w(s)$ be a smooth function satisfying $w(s)=1$ for $s<1$ and $w(s)=0$ for $s \geq 2$. For given $B>\sqrt{p_{1}^{2}+p_{2}^{2}+\cdots+p_{N}^{2}}$, define $\tilde{\Phi}_{B}\left(T ; s, a_{0}\right) u_{0}^{\mu, \xi}$ by

$$
\left(\tilde{\Phi}_{B}\left(T ; s, a_{0}\right) u_{0}^{\mu, \xi}\right)(x)=e^{\int_{0}^{T} a_{0}(s+\tau) d \tau} \cdot \int u_{0}^{\mu, \xi}(x-y) m_{B}(y, d y)
$$

and define $\Phi_{B}\left(T ; s, a_{0}, \mu, \xi\right)$ by

$$
\left(\Phi_{B}\left(T ; s, a_{0}, \mu, \xi\right) u_{0}\right)(x)=e^{\mu x \cdot \xi}\left(\tilde{\Phi}_{B}\left(T, s, a_{0}\right) u_{0}^{\mu, \xi}\right)(x),
$$

where $m_{B}(y, d y)=w(\|y\| / B) m(y, d y)$ and $u_{0}^{\mu, \xi}(x)=e^{-\mu x \cdot \xi} u_{0}(x)\left(u_{0} \in X_{L}\right)$. Similar to $\Phi\left(T ; s, a_{0}, \mu, \xi\right), \Phi_{B}\left(T ; s, a_{0}, \mu, \xi\right): X_{L} \rightarrow X_{L}$ is compact and strongly monotone. Let $\lambda_{T, B}\left(s, a_{0}, \mu, \xi\right)$ be the principal eigenvalue of $\Phi_{B}\left(T, s, a_{0}, \mu, \xi\right)$. Then

$$
\frac{1}{T} \ln \lambda_{T, B}\left(s, a_{0}, \mu, \xi\right)=\frac{1}{T}\left(\int_{0}^{T} a_{0}(s+\tau)+\ln \int e^{\mu y \cdot \xi} m_{B}(y, d y)\right) .
$$

Fix $T, B$, and $a_{0}$. For $\mu>0$, let

$$
\Psi(s, \mu, \xi)=\frac{1}{T} \frac{\ln \lambda_{T, B}\left(s, a_{0}, \mu, \xi\right)}{\mu}
$$

and

$$
\psi(s, \mu, \xi)=\frac{\partial}{\partial \mu}(\mu \Psi(s, \mu, \xi)) .
$$

Then

$$
\Psi(s, \mu, \xi)=\frac{1}{T} \frac{\int_{s}^{T+s} a_{0}(\tau) d \tau+\ln \int e^{\mu y \cdot \xi} m_{B}(y, d y)}{\mu}
$$

and

$$
\psi(s, \mu, \xi)=\frac{1}{T} \frac{\int e^{\mu y \cdot \xi} y \cdot \xi m_{B}(y, d y)}{\int e^{\mu y \cdot \xi} m_{B}(y, d y)} .
$$

Note that $\psi(s, \mu, \xi)$ is independent of $s$. We may then write it as $\psi(\mu, \xi)$. By direct computation,

$$
\psi_{\mu}(\mu, \xi)=\frac{1}{T} \frac{\int e^{\mu y \cdot \xi}(y \cdot \xi)^{2} m_{B}(y, d y) \int e^{\mu y \cdot \xi} m_{B}(y, d y)-\left(\int e^{\mu y \cdot \xi} y \cdot \xi m_{B}(y, d y)\right)^{2}}{\left(\int e^{\mu y \cdot \xi} m_{B}(y, d y)\right)^{2}} .
$$

It then follows from the Hölder inequality that

$$
\psi_{\mu}(\mu, \xi)>0 \text {. }
$$

We also have

$$
\begin{aligned}
& \Psi_{\mu}(s, \mu, \xi)=\frac{1}{\mu}[\psi(\mu, \xi)-\Psi(s, \mu, \xi)], \\
& \frac{\partial}{\partial \mu}\left(\mu^{2} \Psi_{\mu}(s, \mu, \xi)\right)=\mu \frac{\partial}{\partial \mu} \psi(\mu, \xi)>0 .
\end{aligned}
$$

By the properties of fundamental solutions for parabolic equations (see [16]) and the almost periodicity of (1.1) in $t$, we have

$$
\left\|\Phi_{B}\left(T, s, a_{0}, \mu, \xi\right) u_{0}-\Phi\left(T, s, a_{0}, \mu, \xi\right) u_{0}\right\|_{X_{L}} \rightarrow 0
$$


as $B \rightarrow \infty$ uniformly with respect to $s \in \mathbb{R}, \mu$ in bounded subsets of $[0, \infty)$, $\xi \in S^{N-1}$, and $u_{0} \in X_{L}$ with $\left\|u_{0}\right\|_{X_{L}}=1$. This together with (4.5), (4.8), (4.11), (4.14), (4.15), and (4.16) implies that for $B \gg 1$, there is a unique $\mu_{B}^{*}\left(T, s, a_{0}, \xi\right)>0$ such that $\Psi_{\mu}\left(s, \mu_{B}^{*}\left(T, s, a_{0}, \xi\right), \xi\right)=0$ and

$$
\frac{1}{T} \frac{\ln \lambda_{T, B}\left(s, a_{0}, \mu_{B}^{*}\left(T, s, a_{0}, \xi\right), \xi\right)}{\mu_{B}^{*}\left(T, s, a_{0}, \xi\right)}=\inf _{\mu>0} \frac{1}{T} \frac{\ln \lambda_{T, B}\left(s, a_{0}, \mu, \xi\right)}{\mu} .
$$

Moreover, Lemma 4.4 follows.

Lemma 4.4. Let $\epsilon>0$ and $T \geq 1$ be as in Lemma 4.3. Then there is $B \geq 1$ such that

$$
\begin{gathered}
\mu_{B}^{*}\left(T, s, a_{0}, \xi\right) \in\left(\mu^{*}-\epsilon, \mu^{*}+\epsilon\right), \\
\frac{1}{T} \frac{\ln \lambda_{T, B}\left(s, a_{0}, \mu_{B}^{*}\left(T, s, a_{0}, \xi\right), \xi\right)}{\mu_{B}^{*}\left(T, s, a_{0}, \xi\right)} \in\left(\frac{\lambda\left(\mu^{*} ; a_{0}\right)}{\mu^{*}}-\epsilon, \frac{\lambda\left(\mu^{*} ; a_{0}\right)}{\mu^{*}}+\epsilon\right)
\end{gathered}
$$

for all $s \in \mathbb{R}$ and $\xi \in S^{N-1}$. Moreover, there is $\mu_{T, B} \in\left(\mu^{*}-\epsilon, \mu^{*}+\epsilon\right)$ such that

$$
\mu_{T, B}<\mu_{B}^{*}\left(T, s, a_{0}, \xi\right) \quad \text { for all } s \in \mathbb{R} \quad \text { and } \xi \in S^{N-1} .
$$

The next lemma will play a crucial role in the proof of Theorem 4.1 .

Lemma 4.5. Assume that there is an almost periodic function $a_{0}(\cdot)$ such that $\lambda\left(a_{0}\right)>0$ and $f(t, x, u) \geq a_{0}(t) u$ for $t \in \mathbb{R}, x \in \mathbb{R}^{N}$, and $0 \leq u \ll 1$. For any $\epsilon>0$ and $\xi \in S^{N-1}$, there are $T \geq 1$ and $v_{0} \in X_{1}^{+}(\xi)$ such that

$$
u\left(T+s, x ; s, y, v_{0}\right) \geq v_{0}(x-c \xi T)
$$

for all $x, y \in \mathbb{R}^{N}, s \in \mathbb{R}$, and $c<\inf _{\mu>0} \frac{\lambda\left(\mu ; a_{0}\right)}{\mu}-\epsilon\left(=\frac{\lambda\left(\mu^{*} ; a_{0}\right)}{\mu^{*}}-\epsilon\right)$.

Proof. We prove the lemma by modifying the arguments in [36], [34] or [66] for the time periodic case.

First of all, take any $\xi \in S^{N-1}$ and fix it. By Lemma 4.4, for any $\epsilon>0$, there are $T \geq 1$ and $B \geq 1$ such that $\mu_{B}^{*}\left(T, s, a_{0}, \xi\right) \in\left(\mu^{*}-\epsilon, \mu^{*}+\epsilon\right)$ for all $s \in \mathbb{R}$ and there is $\mu_{T, B} \in\left(\mu^{*}-\epsilon, \mu^{*}+\epsilon\right)$ such that $\mu_{T, B}<\mu_{B}^{*}\left(T, s, a_{0}, \xi\right)$ for all $s \in \mathbb{R}$. Then

$$
\frac{1}{T} \frac{\ln \lambda_{T, B}\left(s, a_{0}, \mu_{T, B}, \xi\right)}{\mu_{T, B}}>\frac{\lambda\left(\mu^{*} ; a_{0}\right)}{\mu^{*}}-\epsilon \text { for all } s \in \mathbb{R} .
$$

Fix $T, B$, and $a_{0}$. Let $\Psi(s, \mu, \xi)$ and $\psi(s, \mu, \xi)$ be as in (4.12) and (4.13). Recall that $\psi(s, \mu, \xi)$ is independent of $s$, and we write it as $\psi(\mu, \xi)$. By (4.15) and (4.16),

$$
\psi\left(\mu_{B}^{*}\left(T, s, a_{0}\right), \xi\right)=\Psi\left(s, \mu_{B}^{*}\left(T, s, a_{0}, \xi\right), \xi\right)
$$

and

$$
\psi(\mu, \xi)<\Psi(s, \mu, \xi) \text { for } \mu<\mu_{B}^{*}\left(T, s, a_{0}, \xi\right) .
$$

Note that $\frac{\partial \psi(\mu, \xi)}{\partial \mu}$ for $\mu \in\left[\mu_{-}^{*}, \mu_{+}^{*}\right]$ is bounded above by a constant independent of $T \geq 1$ and $B \geq 1$. Let $\mu=\mu_{T, B}$ in the following. Then there is a constant $M_{0}>0$ (independent of $\epsilon$ ) such that

$$
\psi(\mu, \xi)>\frac{\lambda\left(\mu^{*} ; a_{0}\right)}{\mu^{*}}-M_{0} \epsilon
$$

Let $0<\gamma \ll 1$ be such that

$$
\int e^{\mu y \cdot \xi} \cos (\gamma y \cdot \xi) m_{B}(y, d y)>0 .
$$


Let

$$
z(\gamma)=\frac{1}{\gamma} \tan ^{-1} \frac{\int e^{\mu y \cdot \xi} \sin (\gamma y \cdot \xi) m_{B}(y, d y)}{\int e^{\mu y \cdot \xi} \cos (\gamma y \cdot \xi) m_{B}(y, d x)}
$$

It is not difficult to prove that

$$
\lim _{\gamma \rightarrow 0} z(\gamma)=T \psi(\mu, \xi)
$$

Let $0<\gamma \ll 1$ be such that $z(\gamma)>0$ and

$$
\gamma(B+z(\gamma))<\pi
$$

For $0<\delta<u_{\text {inf }}^{+}$, define

$$
\tilde{v}(r)=\left\{\begin{array}{l}
\delta e^{-\mu r} \sin (\gamma r) \quad \text { for } \quad 0 \leq r \leq \pi / \gamma \\
0 \quad \text { otherwise }
\end{array}\right.
$$

and

$$
v(x)=\tilde{v}(x \cdot \xi)
$$

Choose $0<\delta \ll 1$ such that

$$
0 \leq \tilde{\Phi}\left(t ; s, a_{0}\right) v_{\delta} \leq \beta \quad \text { for } \quad 0 \leq t \leq T, \quad s \in \mathbb{R},
$$

where $v_{\delta}(x) \equiv \delta$ and $\beta>0$ is such that

$$
f(t, x, u) \geq a_{0}(t) u \quad \text { for } \quad t \in \mathbb{R}, x \in \mathbb{R}^{N}, 0 \leq u \leq \beta .
$$

Then by the comparison principle for parabolic equations,

$$
u\left(t+s, \cdot ; s, y, v_{0}\right) \geq \tilde{\Phi}\left(t ; s, a_{0}\right) v_{0} \geq \tilde{\Phi}_{B}\left(t ; s, a_{0}\right) v_{0}
$$

for $0 \leq t \leq T, s \in \mathbb{R}, y \in \mathbb{R}^{N}$, and any $v_{0} \in X$ with $0 \leq v_{0} \leq \delta$.

Note that

$$
\tilde{\Phi}_{B}\left(T ; s, a_{0}\right) v(\cdot+r \xi+z(\gamma) \xi)(0)=e^{\int_{0}^{T} a_{0}(s+\tau) d \tau} \cdot \int v(-y+r \xi+z(\gamma) \xi) m_{B}(y, d y) .
$$

When $0 \leq r \leq \pi / \gamma$ and $\|y\| \leq B,-\pi / \gamma \leq-y \cdot \xi+r+z(\gamma) \leq 2 \pi / \gamma$. Hence

$$
v(-y+r \xi+z(\gamma) \xi) \geq \delta e^{-\mu(-y \cdot \xi+r+z(\gamma))} \sin (\gamma(-y \cdot \xi+r+z(\gamma)))
$$

for $0 \leq r \leq \pi / \gamma$ and $\|y\| \leq B$. This implies that

$$
\begin{aligned}
& \tilde{\Phi}_{B}\left(T ; s, a_{0}\right) v(\cdot+r \xi+z(\gamma) \xi)(0) \\
& \geq \delta \cdot e^{\int_{0}^{T} a_{0}(s+\tau) d \tau} \cdot \int e^{-\mu(-y \cdot \xi+r+z(\gamma))} \sin (\gamma(-y \cdot \xi+r+z(\gamma))) m_{B}(y, d y) \\
& =\delta \cdot e^{\int_{0}^{T} a_{0}(s+\tau) d \tau} e^{-\mu r} e^{-\mu z(\gamma)} \cdot\left(\sin (\gamma(r+z(\gamma))) \int e^{\mu y \cdot \xi} \cos (\gamma y \cdot \xi) m_{B}(y, d y)\right. \\
& \left.\quad-\cos (\gamma(r+z(\gamma))) \int e^{\mu y \cdot \xi} \sin (\gamma y \cdot \xi) m_{B}(y, d y)\right) \\
& =\delta \cdot e^{\int_{0}^{T} a_{0}(s+\tau) d \tau} e^{-\mu r} e^{-\mu z(\gamma)} \cdot \int e^{\mu y \cdot \xi} \cos (\gamma y \cdot \xi) m_{B}(y, d y)(\sin (\gamma(r+z(\gamma))) \\
& \quad-\tan (\gamma z(\gamma)) \cos (\gamma(r+z(\gamma)))) \\
& =e^{-\mu z(\gamma)} \sec (\gamma z(\gamma)) v(r \xi) e^{\int_{0}^{T} a_{0}(s+\tau) d \tau} \cdot \int e^{\mu y \cdot \xi} \cos (\gamma y \cdot \xi) m_{B}(y, d y)
\end{aligned}
$$

for $0 \leq r \leq \pi / \gamma$ and $\|y\| \leq B$. 
Note that

$$
\begin{gathered}
\lim _{\gamma \rightarrow 0} e^{-\mu z(\gamma)}=e^{-\mu T \psi(\mu ; \xi)}, \\
\lim _{\gamma \rightarrow 0} e^{\int_{0}^{T} a_{0}(s+\tau) d s} \cdot \int e^{\mu y \cdot \xi} \cos (\gamma y \cdot \xi) m_{B}(y, d y)=e^{\mu T \Psi(s, \mu ; \xi)} .
\end{gathered}
$$

Hence

$$
\begin{aligned}
& \lim _{\gamma \rightarrow 0} e^{-\mu z(\gamma)} \sec (\gamma z(\gamma)) e^{\int_{0}^{T} a_{0}(s+\tau) d s} \cdot \int e^{\mu y \cdot \xi} \cos (\gamma y \cdot \xi) m_{B}(y, d y) \\
& =e^{\mu T(\Psi(s, \mu ; \xi)-\psi(\mu ; \xi))}>1
\end{aligned}
$$

uniformly in $s \in \mathbb{R}$. This, together with $v(r \xi)=0$ for $r \leq 0$ or $r \geq \pi / \gamma$, implies that for $0<\gamma \ll 1$,

$$
\tilde{\Phi}_{B}\left(T, s, a_{0}\right) v(\cdot+r \xi+z(\gamma) \xi)(0) \geq v(r \xi)
$$

for all $s \in \mathbb{R}$ and $r \in \mathbb{R}$.

Observe that $\tilde{v}$ attains its maximum at a unique point $\bar{r}=\gamma^{-1} \tan ^{-1}(\gamma / \mu)$. Define

$$
\tilde{v}_{0}(r)=\left\{\begin{array}{l}
\tilde{v}(\bar{r}) \quad \text { for } \quad r \leq-\pi / \gamma+\bar{r} \\
\tilde{v}(r+\pi / \gamma) \text { for } r \geq-\pi / \gamma+\bar{r}
\end{array}\right.
$$

and

$$
v_{0}(x)=\tilde{v}_{0}(x \cdot \xi)
$$

Then

$$
\tilde{\Phi}_{B}\left(T, s, a_{0}\right) v_{0}(\cdot)(x) \geq v_{0}(x-z(\gamma) \xi)
$$

for $0<\gamma \ll 1$ and all $x \in \mathbb{R}^{N}$.

Recall that

$$
\lim _{\gamma \rightarrow 0} z(\gamma)=T \psi(\mu, \xi)>T\left(\frac{\lambda\left(\mu^{*}, a_{0}\right)}{\mu^{*}}-M_{0} \epsilon\right) .
$$

Then

$$
u\left(T+s, x ; s, y, v_{0}\right) \geq \tilde{\Phi}_{B}\left(T, s, a_{0}\right) v_{0}(\cdot)(x) \geq v_{0}(x-c \xi T)
$$

for all $s \in \mathbb{R}$ and $x, y \in \mathbb{R}^{N}$, and $c<\frac{\lambda\left(\mu^{*} ; a_{0}\right)}{\mu^{*}}-M_{0} \epsilon$. The lemma is thus proved by the independence of $M_{0}$ on $\epsilon$.

We now prove Theorem 4.1

Proof of Theorem 4.1. (1) It is a special case of Theorem 5.1 (1). See the next section for the proof of Theorem 5.1 (1).

(2) First of all, take any $\xi \in S^{N-1}$ and fix it. For given $\epsilon>0$, let $T$ and $v_{0}$ be as in Lemma 4.5. Then

$$
u\left(T+s, x ; s, y, v_{0}\right) \geq v_{0}(x-c \xi T) \quad \text { for } \quad x, y \in \mathbb{R}^{N}, \quad s \in \mathbb{R} .
$$

This implies that

$$
\begin{aligned}
u\left(s+2 T, x ; s, y, v_{0}\right) & \geq u\left(s+2 T, x ; T+s, y, v_{0}(\cdot-c \xi T)\right) \\
& =u\left(s+2 T, x-c \xi T ; T+s, y+c \xi T, v_{0}(\cdot)\right) \\
& \geq v_{0}(x-2 c \xi T) \quad \text { for } \quad s \in \mathbb{R}, x, y \in \mathbb{R}^{N} .
\end{aligned}
$$

By induction, we have

$$
u\left(s+n T, x ; s, y, v_{0}\right) \geq v_{0}(x-c n \xi T) \quad \text { for } \quad s \in \mathbb{R}, x, y \in \mathbb{R}^{N}, n=1,2, \cdots .
$$


This together with Proposition 3.7 implies that for any $\epsilon>0$ and $c<\inf _{\mu>0} \frac{\lambda\left(\mu ; a_{0}\right)}{\mu}$ $-\epsilon$,

$$
\lim _{x \cdot \xi \leq c t, t \rightarrow \infty}\left(u\left(t+s, x ; s, y, v_{0}\right)-u^{+}(t+s, x+y)\right)=0
$$

uniformly in $s \in \mathbb{R}$. Therefore, by Proposition 3.7 $c_{\text {inf }}^{*}(\xi) \geq \inf _{\mu>0} \frac{\lambda\left(\mu ; a_{0}\right)}{\mu}-\epsilon$ and then $c_{\text {inf }}^{*}(\xi) \geq \inf _{\mu>0} \frac{\lambda\left(\mu ; a_{0}\right)}{\mu}$.

Now assume that $f_{u}(t, x, 0) \equiv f_{u}(t, 0,0)$. Let $a_{0}(t)=f(t, 0,0)$. Then for any $0<\delta \ll 1$,

$$
f(t, x, u) \geq\left(a_{0}(t)-\delta\right) u \quad \text { for } \quad t \in \mathbb{R}, x \in \mathbb{R}^{N}, 0 \leq u \ll 1 .
$$

By the above arguments,

$$
c_{\mathrm{inf}}^{*}(\xi) \geq c_{l}\left(\xi ; a_{0}-\delta\right) .
$$

By Theorem 2.7. $c_{l}\left(\xi ; a_{0}-\delta\right) \rightarrow c_{l}\left(\xi ; a_{0}\right)=c_{l}(\xi)$ as $\delta \rightarrow 0$. We then have

$$
c_{\text {inf }}^{*}(\xi) \geq c_{l}(\xi) \text {. }
$$

(3) Assume that $f(t, x, u) \leq f_{u}(t, x, 0) u$ for $t \in \mathbb{R}, x \in \mathbb{R}^{N}$, and $u \geq 0$, and $f_{u}(t, x, 0) \equiv f_{u}(t, 0,0)$. By $(1)$, we have

$$
c_{\mathrm{sup}}^{*}(\xi) \leq \inf _{\mu>0} \frac{\lambda\left(\mu ; a_{0}\right)}{\mu} \quad \text { for any } \quad \xi \in S^{N-1} .
$$

By (2),

We then must have

$$
c_{\text {inf }}^{*}(\xi) \geq c_{l}(\xi) \quad \text { for any } \quad \xi \in S^{N-1}
$$

$$
c_{\text {inf }}^{*}(\xi)=c_{\text {sup }}^{*}(\xi)=c_{l}(\xi) \quad \text { for any } \quad \xi \in S^{N-1} .
$$

Proof of Theorem 4.2. (1) It is a special case of Theorem 5.2 (1). See the next section for the proof of Theorem 5.2 (1).

(2) We prove it by modifying the arguments in [45.

First of all, let $c<\frac{\lambda\left(\mu, \xi ; a_{0}\right)}{\mu}$ be fixed. Take a $\delta>0$ such that

$$
\lambda\left(\tilde{a}_{0}\right)>0, \quad c<\frac{\lambda\left(\mu, \xi ; \tilde{a}_{0}\right)}{\mu}, \quad \text { and } \mu<\tilde{\mu}^{*},
$$

where $\tilde{a}_{0}(t)=a_{0}(t)-\delta$ and $\tilde{\mu}^{*}$ is such that $\frac{\lambda\left(\tilde{\mu}^{*}, \xi ; \tilde{a}_{0}\right)}{\tilde{\mu}^{*}}=\inf _{\mu>0} \frac{\lambda\left(\mu, \xi ; \tilde{a}_{0}\right)}{\mu}$ (this is possible because of the continuity of $\lambda\left(\mu, \xi ; a_{0}\right)$ in $\left.a_{0}\right)$. Let $\beta>0$ be such that

$$
f(t, x, u) \geq a_{0}(t) u \quad \text { for } \quad t \in \mathbb{R}, x \in \mathbb{R}^{N}, 0 \leq u \leq \beta .
$$

For any given constant $M_{0}$, let $\tilde{f}(t, u)=u\left(a_{0}(t)-M_{0} u\right)$. Then (1.1) with $f$ being replaced by $\tilde{f}$ satisfies $(\mathrm{H} 1)$ and $(\mathrm{H} 2)$. Let $\tilde{u}^{+}(t, x)$ be the positive solution of (1.1) with $f$ being replaced by $\tilde{f}$ satisfying the fact that $\tilde{u}^{+}(t, x)$ is almost periodic in $t$ and periodic in $x_{j}$ with period $p_{j}(j=1,2, \cdots, N)$. Choose $M_{0}$ sufficiently large so that

$$
0<\tilde{u}^{+}(t, x)<\beta \quad \text { for } \quad t \in \mathbb{R}, x \in \mathbb{R}^{N} .
$$

Then there is $\tilde{\beta}$ with $0<\tilde{\beta}<\beta$ such that

$$
f(t, x, u) \geq \tilde{f}(t, u) \geq \tilde{a}_{0}(t) u \quad \text { for } \quad t \in \mathbb{R}, x \in \mathbb{R}^{N}, 0 \leq u \leq \tilde{\beta} .
$$

Moreover, there is $\tilde{u}_{0}^{*}(r)$ such that it is decreasing in $r$,

$$
\tilde{u}_{0}(x) \leq u_{0}(x) \quad \text { and } \quad \tilde{u}_{0}(x)<\tilde{\beta} \quad \text { for } \quad x \in \mathbb{R}^{N},
$$


and

$$
\tilde{u}_{0}(x) \geq \tilde{C} e^{-\mu x \cdot \xi} \quad \text { for } \quad x \cdot \xi \gg 1 \text { and some } \tilde{C}>0,
$$

where $\tilde{u}_{0}(x)=\tilde{u}_{0}^{*}(x \cdot \xi)$. Clearly, $\tilde{u}_{0} \in X_{2}^{+}(\xi)$.

Note that $\lambda\left(\mu, \xi ; \tilde{a}_{0}\right)$ is independent of $\xi \in S^{N-1}$, and we may then write it as $\lambda\left(\mu ; \tilde{a}_{0}\right)$. Take $\mu_{1}, \mu_{2}$ with $\mu<\mu_{1}<\mu_{2}<\tilde{\mu}^{*}$ such that $c<\frac{\lambda\left(\mu_{2} ; \tilde{a}_{0}\right)}{\mu_{2}}$. Then

$$
\frac{\lambda\left(\mu ; \tilde{a}_{0}\right)}{\mu}>\frac{\lambda\left(\mu_{1} ; \tilde{a}_{0}\right)}{\mu_{1}}>\frac{\lambda\left(\mu_{2} ; \tilde{a}_{0}\right)}{\mu_{2}}>\frac{\lambda\left(\tilde{\mu}^{*} ; \tilde{a}_{0}\right)}{\tilde{\mu}^{*}} .
$$

For any $\epsilon>0$, there is $T>0$ such that

$$
c<\frac{\lambda\left(\mu_{i} ; \tilde{a}_{0}\right)}{\mu_{i}}-\epsilon<\frac{\int_{s}^{T+s} \tilde{a}_{0}^{\mu_{i}, \xi}(\tau) d \tau}{T \mu_{i}}<\frac{\lambda\left(\mu_{i} ; \tilde{a}_{0}\right)}{\mu_{i}}+\epsilon
$$

for $i=1,2$ and all $s \in \mathbb{R}$, and

$$
\frac{\int_{s}^{T+s} \tilde{a}_{0}^{\mu_{1}, \xi}(\tau) d \tau}{T \mu_{1}}>\frac{\int_{s}^{T+s} \tilde{a}_{0}^{\mu_{2}, \xi}(\tau) d \tau}{T \mu_{2}}+\epsilon
$$

for all $s \in \mathbb{R}$.

Let

$$
c_{i}^{n}(s)=\frac{1}{T} \frac{\int_{s+(n-1) T}^{s+n T} \tilde{a}_{0}^{\mu_{i}, \xi}(\tau) d \tau}{\mu_{i}}
$$

and

$$
\phi_{i}^{n}(t)=e^{\int_{s+(n-1) T}^{t}\left(\tilde{a}_{0}^{\mu_{i}, \xi}(\tau)-\mu_{i} c_{i}^{n}(s)\right) d \tau}
$$

for $s \in \mathbb{R}, i=1,2$, and $n=1,2, \cdots$.

Let

$$
\begin{aligned}
\psi_{n}(t, x ; s)= & d_{1} e^{-\mu_{1}\left(x \cdot \xi-\sum_{k=1}^{n-1} c_{2}^{k}(s) T-c_{2}^{n}(s)(t-(n-1) T-s)\right)} \phi_{1}^{n}(t) \\
& -d_{2} e^{-\mu_{2}\left(x \cdot \xi-\sum_{k=1}^{n-1} c_{2}^{k}(s) T-c_{2}^{n}(s)(t-(n-1) T-s)\right)} \phi_{2}^{n}(t)
\end{aligned}
$$

for $x \in \mathbb{R}^{N}, s \in \mathbb{R}, s+(n-1) T \leq t<s+n T$, and $n=1,2, \cdots$, where $d_{1}$ and $d_{2}$ are two positive constants to be determined later.

Note that $c_{2}^{n}(s)<c_{1}^{n}(s)$ for $s \in \mathbb{R}$ and $n=1,2, \cdots$. Then $\psi_{n}(t, x ; s)$ is a subsolution of (4.6) with $a_{0}$ being replaced by $\tilde{a}_{0}$ for $s+(n-1) T<t<s+n T$. Note also that

$$
\begin{gathered}
\psi_{n}(s+n T, x ; s)=\psi_{n+1}(s+n T, x ; s) \quad \text { for } \quad x \in \mathbb{R}^{N}, s \in \mathbb{R}, n=1,2, \cdots . \\
\psi_{n}(t, x ; s)<0 \text { for } x \cdot \xi-\sum_{k=1}^{n-1} c_{2}^{k}(s) T-c_{2}^{n}(s)(t-(n-1) T-s) \ll 0, \text { and } \psi_{n}(t, x ; s) \sim \\
d_{1} e^{-\mu_{1}\left(x \cdot \xi-\sum_{k=1}^{n-1} c_{2}^{k}(s) T-c_{2}^{n}(s)(t-(n-1) T-s)\right)} \phi_{1}^{n}(t) \text { for } \\
x \cdot \xi-\sum_{k=1}^{n-1} c_{2}^{k}(s) T-c_{2}^{n}(s)(t-(n-1) T-s) \gg 1 .
\end{gathered}
$$

It then follows that there are $d_{1}>d_{2}>0$ such that

$$
\begin{gathered}
\tilde{u}_{0}(x) \geq \psi_{1}(s, x ; s), \\
\psi_{n}(t, x ; s) \leq \tilde{\beta}, \quad s+(n-1) T \leq t \leq s+n T
\end{gathered}
$$


for $x \in \mathbb{R}^{N}, s \in \mathbb{R}$, and $n=1,2, \cdots$, and

$$
\psi_{n}\left(t,\left(\sum_{k=1}^{n-1} c_{2}^{k}(s) T+c_{2}^{n}(s)(t-(n-1) T-s)\right) \xi ; s\right)=d_{1} \phi_{1}^{n}(t)-d_{2} \phi_{2}^{n}(t) \geq \delta_{0}
$$

for some $\delta_{0}>0$, any $s \in \mathbb{R}, s+(n-1) T \leq t \leq s+n T, n=1,2, \cdots$.

Let $\tilde{u}\left(t, x ; s, \tilde{u}_{0}\right)$ be the solution of (4.2) with $f$ being replaced by $\tilde{f}$ and with $\tilde{u}\left(s, x ; s, \tilde{u}_{0}\right)=\tilde{u}_{0}(x)$. It then follows that

$$
\beta>\tilde{u}\left(t, x ; s, \tilde{u}_{0}\right) \geq \psi_{n}(t, x ; s) \quad \text { for } \quad s+(n-1) T \leq t \leq s+n T
$$

for $n=1,2, \cdots$ and $s \in \mathbb{R}, x, y \in \mathbb{R}^{N}$.

Then by the comparison principle for parabolic equations, we have

$$
u\left(t, x ; s, y, u_{0}\right) \geq u\left(t, x ; s, y, \tilde{u}_{0}\right) \geq \tilde{u}\left(t, x ; s, \tilde{u}_{0}\right) \geq \delta_{0}
$$

for $s \in \mathbb{R}, y \in \mathbb{R}^{N}, x \cdot \xi \leq \sum_{k=1}^{n-1} c_{2}^{k}(s) T+c_{2}^{n}(s)(t-(n-1) T+s)$ and $s+(n-1) T \leq$ $t \leq s+n T, n=1,2, \cdots$.

By Proposition 3.7, we then have

$$
\liminf _{x \cdot \xi \leq(c-\epsilon) t, t \rightarrow \infty}\left(u\left(t+s, x ; s, y, u_{0}\right)-u^{+}(t+s, x+y)\right)=0
$$

uniformly in $s \in \mathbb{R}$ and $y \in \mathbb{R}^{N}$. This, together with the arbitrariness of $c$ with $c<\frac{\lambda\left(\mu, \xi ; a_{0}\right)}{\mu}$ and the arbitrariness of $\epsilon$, implies that $c_{\inf }\left(u_{0}, \xi\right) \geq \frac{\lambda\left(\mu, \xi ; a_{0}\right)}{\mu}$.

(3) First, by (1),

$$
c_{\text {sup }}\left(u_{0}, \xi ; a_{0}\right) \leq \frac{\lambda\left(\mu, \xi ; a_{0}\right)}{\mu},
$$

where $a_{0}(t)=f_{u}(t, 0,0) \equiv f_{u}(t, x, 0)$.

For any $\delta>0$,

$$
f(t, x, u) \geq\left(f_{u}(t, x, 0)-\delta\right) u \quad \text { for } \quad t \in \mathbb{R}, x \in \mathbb{R}^{N}, 0<u \ll 1 .
$$

Then by (2),

$$
c_{\text {inf }}\left(u_{0}, \xi\right) \geq \frac{\lambda\left(\mu, \xi ; a_{0}-\delta\right)}{\mu} .
$$

Letting $\delta \rightarrow 0$, by Theorem 2.7, we have

$$
c_{\mathrm{sup}}\left(u_{0}, \xi\right)=c_{\mathrm{inf}}\left(u_{0}, \xi\right)=\frac{\lambda\left(\mu, \xi ; a_{0}\right)}{\mu} .
$$

\section{Spreading SPEEDS AND GENERALIZED PROPAGATING SPEEDS IN SOME GENERAL CASE}

In this section, we investigate the spatial spread and front propagation dynamics in some general case.

Throughout this section, we assume that $a_{0}(t, x)$ is some given function which is uniformly almost periodic in $t$, periodic in $x_{j}$ with period $p_{j}(j=1,2, \cdots, N)$, and globally Hölder continuous in $t$ and $x$. $u\left(t, \cdot ; s, y, u_{0}\right)$ denotes the solution of (1.5) with $u\left(s, \cdot ; s, y, u_{0}\right)=u_{0}(\cdot)\left(u_{0} \in X\right)$, and $u\left(t, \cdot ; s, u_{0}\right)=u\left(t, \cdot ; s, 0, u_{0}\right)$. For given $a_{0}(t, x), \mu \geq 0$, and $\xi \in S^{N-1}, \lambda\left(\mu, \xi ; a_{0}\right)$ is the principal Lyapunov exponent of (3.4) and (3.3).$\phi\left(\sigma_{t} a^{\mu, \xi}\right)$ is the associated principal Floquet bundle (see (2.4) in section 2 for a definition), where $a^{\mu, \xi}=\left(\left\{a_{i}^{\mu, \xi}\right\}_{i=1}^{N}, a_{0}^{\mu, \xi}\right)$ (see (3.4) and $\sigma_{t} a^{\mu, \xi}(\cdot, \cdot)=a^{\mu, \xi}(\cdot+t, \cdot) . \kappa\left(\sigma_{t} a^{\mu, \xi}\right)$ is defined as in (2.5) with $b$ being replaced by $\sigma_{t} a^{\mu, \xi} . c_{l}\left(\xi ; a_{0}\right)$ and $c_{l}(\xi)$ are defined in (1.7) and (1.8), respectively.

The main results of this section are stated as follows. 
Theorem 5.1 (Upper and lower bounds and variational principle).

(1) (Upper bound) Assume that $f(t, x, u) \leq a_{0}(t, x) u$ for $t \in \mathbb{R}, x \in \mathbb{R}^{N}$, and $u \geq 0$. Then

$$
c_{\text {sup }}^{*}(\xi) \leq c_{l}\left(\xi ; a_{0}\right) \quad \text { for any } \xi \in S^{N-1} .
$$

(2) (Lower bound) Assume that $a_{i}(t, x) \equiv a_{i}(x)$ and that $\lambda\left(a_{0}\right)>0, f(t, x, u) \geq$ $a_{0}(t, x) u$ for $t \in \mathbb{R}, x \in \mathbb{R}^{N}, 0 \leq u \ll 1$. Assume further that $a_{0}(t, x)$ can be approximated by time periodic functions. Then

$$
c_{\text {inf }}^{*}(\xi) \geq c_{l}\left(\xi ; a_{0}\right) \quad \text { for any } \quad \xi \in S^{N-1} .
$$

In particular, if $a_{i}(t, x) \equiv a_{i}(x)$ and $f_{u}(t, x, 0)$ can be approximated by time periodic functions, then

$$
c_{\text {inf }}^{*}(\xi) \geq c_{l}(\xi) \quad \text { for any } \quad \xi \in S^{N-1} .
$$

(3) (Variational principle/linear determinacy) Assume that $a_{i}(t, x) \equiv a_{i}(x)$ and $f(t, x, u)$ satisfies that $f(t, x, u) \leq f_{u}(t, x, 0) u$ for $t \in \mathbb{R}, x \in \mathbb{R}^{N}$, and $u \geq 0$, and $f_{u}(t, x, 0)$ can be approximated by time periodic functions. Then

$$
c_{\mathrm{inf}}^{*}(\xi)=c_{\mathrm{sup}}^{*}(\xi)=c_{l}(\xi) \quad \text { for any } \quad \xi \in S^{N-1} .
$$

Theorem 5.2 (Generalized propagation).

(1) (Upper bound) Assume that $f(t, x, u) \leq a_{0}(t, x) u$ for $t \in \mathbb{R}, x \in \mathbb{R}^{N}$, and $u \geq 0$. If $u_{0} \in X_{2}^{+}(\xi)$ is such that $u_{0}(x) \leq C e^{-\mu x \cdot \xi}$ for $x \cdot \xi \gg 1$ and some $C>0$ and $\mu>0$, then

$$
c_{\text {sup }}\left(u_{0}, \xi\right) \leq \frac{\lambda(\mu, \xi)}{\mu} .
$$

(2) (Lower bound) Assume that $a_{i}(t, x) \equiv a_{i}(x)(i=1,2, \cdots, N)$ and that $\lambda\left(a_{0}\right)>0, f(t, x, u) \geq a_{0}(t, x) u$ for $t \in \mathbb{R}, x \in \mathbb{R}^{N}, 0 \leq u \ll 1$. Assume further that $a_{0}(t, x)$ can be approximated by time periodic functions. If $u_{0} \in X_{2}^{+}(\xi)$ is such that $u_{0}(x) \geq C e^{-\mu x \cdot \xi}$ for $x \cdot \xi \gg 1$ and some $C>0$ and $0<\mu<\mu^{*}$, where $\mu^{*}$ is such that $\frac{\lambda\left(\mu^{*}, \xi ; a_{0}\right)}{\mu^{*}}=\inf _{\mu>0} \frac{\lambda\left(\mu, \xi ; a_{0}\right)}{\mu}$, then

$$
c_{\text {inf }}\left(u_{0}, \xi\right) \geq \frac{\lambda\left(\mu, \xi ; a_{0}\right)}{\mu} .
$$

(3) (Front solutions of constant speeds) Assume that $a_{i}(t, x) \equiv a_{i}(x)(i=$ $1,2, \cdots, N)$ and $f(t, x, u) \leq f_{u}(t, x, 0) u$ for $t \in \mathbb{R}, x \in \mathbb{R}^{N}$, and $u \geq 0$, and that $a_{0}(t, x)=f_{u}(t, x, 0)$ can be approximated by time periodic functions. If $u_{0} \in X_{2}^{+}(\xi)$ is such that $C_{1} e^{-\mu x \cdot \xi} \leq u_{0}(x) \leq C_{2} e^{-\mu x \cdot \xi}$ for $x \cdot \xi \gg 1$ and some $C_{1}, C_{2}>0$ and $0<\mu<\mu^{*}$, then

$$
c_{\text {inf }}\left(u_{0}, \xi\right)=u_{\text {sup }}\left(u_{0}, \xi\right)=\frac{\lambda\left(\mu, \xi ; a_{0}\right)}{\mu} .
$$

Hence for any $c \geq c_{l}(\xi)$, there is a front solution of (1.1) in the direction of $\xi$ which propagates at the speed $c$ and there is no front solution in the direction of $\xi$ which propagates slower than $c_{l}(\xi)$.

Observe that $f(t, x, u)=u(a(x)-b(t, x) u), f(t+T, x, u)=f(t, x, u)$, and $f(t, x, u) \equiv f(x, u)$ are examples with the property that $f_{u}(t, x, 0)$ can be approximated by time periodic functions. 
Proof of Theorem [5.1. (1) Assume that $f(t, x, u) \leq a_{0}(t, x) u$ for $t \in \mathbb{R}, x \in \mathbb{R}^{N}$, and $u \geq 0$.

Given any $\mu>0$ and $u_{0} \in X_{1}^{+}(\xi)$, there is $\alpha_{0}>0$ such that

$$
u_{0}(x) \leq \alpha_{0} e^{-\mu x \cdot \xi} \phi\left(\sigma_{s} a^{\mu, \xi}\right)(x)
$$

for $x \in \mathbb{R}^{N}$ and $s \in \mathbb{R}$. It then follows from the comparison principle for parabolic equations and Theorem 2.8 that

$$
u\left(t, x ; s, u_{0}\right) \leq \alpha_{0} e^{-\mu\left(x \cdot \xi-\frac{\int_{s}^{t} \kappa\left(\sigma_{\tau} a^{\mu, \xi}\right) d \tau}{\mu}\right)} \phi\left(\sigma_{t} a^{\mu, \xi}\right)(x)
$$

for $s \in \mathbb{R}, t>s$, and $x \in \mathbb{R}^{N}$.

Note that

$$
\lambda\left(\mu, \xi ; a_{0}\right)=\lim _{t-s \rightarrow \infty} \frac{1}{t-s} \int_{s}^{t} \kappa\left(\sigma_{\tau} a^{\mu, \xi}\right) d \tau
$$

(see Theorem [2.4). Hence for any $\epsilon>0$,

$$
u\left(t+s, x ; s, u_{0}\right) \leq e^{-\mu\left(x \cdot \xi-\frac{\lambda(\mu, \xi)+\epsilon}{\mu} t\right)} \phi\left(\sigma_{t+s} a^{\mu, \xi}\right)(x)
$$

for $t \gg 1$ and $s \in \mathbb{R}$. This implies that for any $c>\frac{\lambda\left(\mu, \xi ; a_{0}\right)}{\mu}$,

$$
\limsup _{x \cdot \xi \geq c t, t \rightarrow \infty} u\left(t+s, x ; s, u_{0}\right)=0
$$

uniformly in $s \in \mathbb{R}$. Therefore $c_{\text {sup }}^{*}(\xi) \leq \frac{\lambda(\mu, \xi ; a)}{\mu}$ for any $\mu>0$ and then $c_{\text {sup }}^{*}(\xi) \leq$ $\inf _{\mu>0} \frac{\lambda\left(\mu, \xi ; a_{0}\right)}{\mu}$.

(2) First let $0<\beta<1$ be such that

$$
f(t, x, u) \geq a_{0}(t, x) u \quad \text { for } \quad t \in \mathbb{R}, x \in \mathbb{R}^{N}, 0 \leq u \leq \beta .
$$

Assume that $a_{0}^{n}(t, x)(n=1,2, \cdots)$ are periodic in $t$ with period $T_{n}$ and that

$$
\lim _{n \rightarrow \infty} \sup _{t \in \mathbb{R}, x \in \mathbb{R}^{N}}\left|a_{0}^{n}(t, x)-a_{0}(t, x)\right|=0 .
$$

Without loss of generality, we may assume that

$$
a_{0}^{n}(t, x) \leq a_{0}(t, x) \quad \text { for } \quad t \in \mathbb{R}, \quad x \in \mathbb{R}^{N}, n=1,2, \cdots .
$$

Define $f_{n}(t, x)=u\left(a_{0}^{n}(t, x)-M_{0} u\right)$, where $M_{0}$ is some positive number to be determined later. Then

$$
f(t, x, u) \geq f_{n}(t, x, u) \quad \text { for } \quad t \in \mathbb{R}, x \in \mathbb{R}^{N}, 0 \leq u \leq \beta,
$$

and by Theorem 2.7

$$
\lambda\left(0, \xi ; a_{0}^{n}\right)>0 \text { for } n \gg 1 .
$$

It follows from the arguments in 61] that (1.1), with $f$ being replaced by $f_{n}$, satisfies (H1) and (H2). Let $u_{n}^{+}(t, x)$ be the positive solution of (1.1) with $f$ being replaced by $f_{n}$ satisfying the fact that $u_{n}^{+}(t, x)$ is almost periodic in $t$ and periodic in $x_{j}$ with period $p_{j}(j=1,2, \cdots, N)$. Take $M_{0}>0$ to be sufficiently large so that

$$
0<u_{n}^{+}(t, x)<\beta \quad \text { for } \quad t \in \mathbb{R}, x \in \mathbb{R}^{N}, n \gg 1 .
$$

Let $c_{n}^{*}(\xi)$ be the spreading speed of (1.1) with $f(t, x, u)$ being replaced by $f_{n}(t, x, u)$. Then by Proposition 3.7 and the comparison principle for parabolic equations,

$$
c_{\mathrm{inf}}^{*}(\xi) \geq c_{n}^{*}(\xi) \text { for } \quad \xi \in S^{N-1}, n \gg 1 .
$$


Now by the theory developed in [65, [66, there is a bounded sequence $\left\{\mu_{n}\right\}$ such that

$$
c_{n}^{*}(\xi)=\inf _{\mu>0} \frac{\lambda\left(\mu, \xi ; a_{0}^{n}\right)}{\mu}=\frac{\lambda\left(\mu_{n}, \xi ; a_{0}^{n}\right)}{\mu_{n}} .
$$

Without loss of generality, assume that $\mu_{n} \rightarrow \mu_{0}$. By the continuity of $\lambda\left(\mu, \xi ; a_{0}^{n}\right)$ (see Theorem 2.7) in $a_{0}^{n}$ and $\lambda\left(0, \xi ; a_{0}\right)>0$ (see (H2)), we must have $\mu_{0} \neq 0$. Hence by Theorem 2.7

$$
\lim _{n \rightarrow \infty} \lambda\left(\mu_{n}, \xi ; a_{0}^{n}\right)=\lambda\left(\mu_{0}, \xi ; a_{0}\right)
$$

It then follows that

$$
c_{\mathrm{inf}}^{*}(\xi) \geq \frac{\lambda\left(\mu_{0}, \xi ; a_{0}\right)}{\mu_{0}} \geq \inf _{\mu>0} \frac{\lambda\left(\mu, \xi ; a_{0}\right)}{\mu} .
$$

Finally assume that $a_{i}(t, x) \equiv a_{i}(x)(i=1,2, \cdots, N)$ and that $f_{u}(t, x, 0)$ can be approximated by time periodic functions. Note that for any $\delta>0$,

$$
f(t, x, u) \geq\left(f_{u}(t, x, 0)-\delta\right) u \quad \text { for } \quad t \in \mathbb{R}, x \in \mathbb{R}^{N}, 0 \leq u \ll 1 .
$$

Let $a_{0}=f_{u}(t, x, 0)$. Then by the above arguments, $c_{\mathrm{inf}}^{*}(\xi) \geq c_{l}\left(\xi ; a_{0}-\delta\right)$ for any $\xi \in S^{N-1}$ and $\delta>0$. Letting $\delta \rightarrow 0$, by Theorem 2.7 we have

$$
c_{\mathrm{inf}}^{*} \geq c_{l}(\xi) \text { for any } \xi \in S^{N-1} .
$$

(3) It follows from (1) and (2).

Proof of Theorem 5.2. (1) By the assumption on $u_{0}$, there is $\alpha>0$ such that

$$
u_{0}(x-y) \leq \alpha e^{-\mu x \cdot \xi} \phi\left(\sigma_{s} a^{\mu, \xi}\right)(x) \text { for } \quad x \in \mathbb{R}^{N}
$$

for any $s \in \mathbb{R}$ and $y \in D$. It then follows from Theorem 2.8, the comparison principle for parabolic equations, and the assumption $f(t, x, u) \leq a_{0}(t, x) u$ for $t \in \mathbb{R}, x \in \mathbb{R}^{N}$ and $u \geq 0$ that

$u\left(t, x ; s, y, u_{0}\right)=u\left(t, x+y ; s, u_{0}(\cdot-y)\right) \leq \alpha e^{-\mu\left((x+y) \cdot \xi-\frac{\int_{s}^{t} \kappa\left(\sigma_{\tau} a^{\mu, \xi}\right) d \tau}{\mu}\right)} \phi\left(\sigma_{t} a^{\mu, \xi}\right)(x+y)$ for $t \geq s, x \in \mathbb{R}^{N}$, and $y \in D$. Note that

$$
\lim _{t-s \rightarrow \infty} \frac{1}{t-s} \int_{s}^{t} \kappa\left(\sigma_{\tau} a^{\mu, \xi}\right) d \tau=\lambda\left(\mu, \xi ; a_{0}\right)
$$

Hence for any $\epsilon>0$,

$$
\frac{\int_{s}^{t+s} \kappa\left(\sigma_{\tau} a^{\mu, \xi}\right) d \tau}{\mu} \leq\left(\frac{\lambda(\mu, \xi)}{\mu}+\epsilon\right) t
$$

for $t \gg 1$ and any $s \in \mathbb{R}$. This implies that for $c>\frac{\lambda\left(\mu, \xi ; a_{0}\right)}{\mu}$,

$$
\limsup _{x \cdot \xi \geq c t, t \rightarrow \infty} u\left(t+s, x ; s, y, u_{0}\right)=0
$$

uniformly in $s \in \mathbb{R}$ and $y \in D$ (hence $y \in \mathbb{R}^{N}$ by the periodicity (1.1) in $x$ ). Therefore $c_{\text {sup }}\left(\xi, u_{0}\right) \leq \frac{\lambda\left(\mu, \xi ; a_{0}\right)}{\mu}$.

(2) If $\frac{\lambda\left(\mu, \xi ; a_{0}\right)}{\mu}=c_{\mathrm{inf}}^{*}(\xi)$, it follows from Proposition 3.5 that $c_{\mathrm{inf}}\left(u_{0}, \xi\right) \geq$ $c_{\mathrm{inf}}^{*}(\xi)=\frac{\lambda\left(\mu, \xi ; a_{0}\right)}{\mu}$. 
Assume $\frac{\lambda\left(\mu, \xi ; a_{0}\right)}{\mu}>c_{\mathrm{inf}}^{*}(\xi)=\frac{\lambda\left(\mu^{*}, \xi ; a_{0}\right)}{\mu^{*}}$ and $\mu<\mu^{*}$. Assume $a_{0}^{n}(t, x)(n=$ $1,2, \cdots)$ are periodic in $t$ with period $T_{n}$ and that

$$
\lim _{n \rightarrow \infty} \sup _{t \in \mathbb{R}, x \in \mathbb{R}^{N}}\left|a_{0}^{n}(t, x)-a_{0}(t, x)\right|=0 .
$$

Without loss of generality, we assume that

$$
a_{0}^{n}(t, x) \leq a_{0}(t, x) \quad \text { for } \quad t \in \mathbb{R}, \quad x \in \mathbb{R}^{N}, n=1,2, \cdots .
$$

Then there are $\mu<\mu_{1}<\mu_{2}<\mu^{*}$ such that

$$
\frac{\lambda\left(\mu, \xi ; a_{0}\right)}{\mu}>c_{1}^{n}:=\frac{\lambda\left(\mu_{1}, \xi ; a_{0}^{n}\right)}{\mu_{1}}>c_{2}^{n}:=\frac{\lambda\left(\mu_{2}, \xi ; a_{0}^{n}\right)}{\mu_{2}}
$$

for $n \gg 1$. Note that $c_{1}^{n}$ and $c_{2}^{n}$ can be made as close to $\frac{\lambda\left(\mu, \xi ; a_{0}\right)}{\mu}$ as we wish by choosing sufficiently large $n$ and proper $\mu_{1}, \mu_{2}$.

Assume that $p \in \mathbb{R}^{N}$ is such that $a_{i}(t, x+p) \equiv a_{i}(t, x)(i=1,2, \cdots, N)$, $a_{0}(t, x+p) \equiv a_{0}(t, x), f(t, x+p, u) \equiv f(t, x, u)$, and $p \cdot \xi>0$. Fix $n$ sufficiently large. Let

$$
\psi^{n}(t+s, x)=d_{1} e^{-\mu_{1}\left(x \cdot \xi-c_{2}^{n} t\right)} \phi^{*}\left(t, x ; \mu_{1}, \xi, a_{0}^{n}\right)-d_{2} e^{-\mu_{2}\left(x \cdot \xi-c_{2}^{n} t\right)} \phi^{*}\left(t, x ; \mu_{2}, \xi, a_{0}^{n}\right),
$$

where $d_{1}, d_{2}$ are positive constants to be determined later, and $\phi^{*}\left(t, x ; \mu_{i}, \xi, a_{0}^{n}\right)$ is a positive principal eigenfunction of (1.4) with $a_{0}$ being replaced by $a_{0}^{n}$ and $T=T_{n}(i=1,2)$. Then by Theorem 2.9, $\psi^{n}(t, x)$ is a sub-solution of (3.4) with $a_{0}$ being replaced by $a_{0}^{n}$. Note that $\psi^{n}(t, x)<0$ for $x \cdot \xi-c_{2}^{n} t \ll 0$ and $\psi^{n}(t, x) \sim d_{1} e^{-\mu_{1}\left(x \cdot \xi-c_{2}^{n} t\right)} \phi_{1}^{n}(t, x)$ for $x \cdot \xi-c_{2}^{n} t \gg 1$. It then follows that there are $d_{1}>d_{2}>0$ such that

$$
u_{0}(\cdot-y-k p) \geq \psi^{n}(s, \cdot)
$$

for all $s \in \mathbb{R}, y \in D, k \in \mathbb{Z}^{+}$,

$$
\psi^{n}(t+s, x)>\delta>0
$$

for $c_{2}^{n} t-p \cdot \xi \leq x \cdot \xi \leq c_{2}^{n} t$ and some $\delta \ll 1$, and

$$
\psi^{n}(t+s, \cdot) \leq \beta, \quad t \geq 0,
$$

for all $s \in \mathbb{R}$, where $\beta>0$ is such that $f(t, x, u) \geq a_{0}(t, x) u$ for $0 \leq u \leq \beta$. It then follows that

$$
u\left(t+s, x-k p \xi ; s, y, u_{0}\right)=u\left(t+s, x ; s, u_{0}(\cdot-y-k p \xi)\right) \geq \psi^{n}(t+s, x)
$$

for $t>0, s \in \mathbb{R}, y \in D, k \in \mathbb{Z}^{+}$. This implies that

$$
u\left(t+s, x ; s, y, u_{0}\right) \geq \delta
$$

for $s \in \mathbb{R}, t>0, y \in D, x \cdot \xi \leq c_{2}^{n} t$.

By (5.1) and Proposition 3.7 for $c<c_{2}^{n}$,

$$
\lim _{x \cdot \xi \leq c t, t \rightarrow \infty}\left(u\left(t+s, x ; s, u_{0}\right)-u^{+}(t+s, x)\right)=0
$$

uniformly in $s \in \mathbb{R}$ and $y \in D$ (hence $\left.y \in \mathbb{R}^{N}\right)$. This implies that $c_{\mathrm{inf}}\left(u_{0}, \xi\right) \geq c_{2}^{n}$ and then $c_{\text {inf }}\left(u_{0}, \xi\right) \geq \frac{\lambda\left(\mu, \xi ; a_{0}\right)}{\mu}$.

(3) First, by (1),

$$
c_{\mathrm{sup}}\left(u_{0}, \xi\right) \leq \frac{\lambda\left(\mu, \xi ; a_{0}\right)}{\mu}
$$


For any $0<\delta \ll 1, f(t, x, u) \geq\left(f_{u}(t, x, 0)-\delta\right) u$ for $t \in \mathbb{R}, x \in \mathbb{R}^{N}$, and $0<u \ll 1$. By $(2)$,

$$
c_{\mathrm{inf}}\left(u_{0}, \xi\right) \geq \frac{\lambda\left(\mu, \xi ; a_{0}-\delta\right)}{\mu} .
$$

Letting $\delta \rightarrow 0$, by Theorem 2.7, we have

$$
c_{\mathrm{inf}}\left(u_{0}, \xi\right) \geq \frac{\lambda\left(\mu, \xi ; a_{0}\right)}{\mu} .
$$

Therefore, we must have

$$
c_{\text {inf }}\left(u_{0}, \xi\right)=c_{\text {sup }}\left(u_{0}, \xi\right)=\frac{\lambda\left(\mu, \xi ; a_{0}\right)}{\mu} .
$$

Next, for any $c \geq c_{l}(\xi)$, choose $u_{0} \in X_{2}^{+}(\xi)$ such that $u_{0}(x)=e^{-\mu x \cdot \xi}$ for $x \cdot \xi \gg 1$ if $c>c_{l}(\xi)$ and choose $u_{0} \in X_{1}^{+}(\xi)$ if $c=c_{l}(\xi)$. Then by the above arguments, Proposition 3.5, and Theorem 5.1 (3), $u\left(t, x ; s, u_{0}\right)$ is a front solution of (1.1) in the direction of $\xi$ with speed $c$. By Proposition 3.5 again, for any $u_{0} \in X_{2}^{+}(\xi)$, $c_{\text {sup }}\left(u_{0}, \xi\right) \geq c_{\text {inf }}\left(u_{0}, \xi\right) \geq c_{\text {inf }}^{*}(\xi)=c_{l}(\xi)$. Hence there is no front solution in the direction of $\xi$ which propagates slower than $c_{l}(\xi)$.

\section{INFLUENCE OF SPATIAL AND TEMPORAL VARIATIONS ON SPREADING SPEEDS}

In this section, we consider the influence of time and space variation on spreading speed.

Throughout this section, $a_{0}(t, x)=f_{u}(t, x, 0)$. We write $\lambda\left(\mu, \xi ; a_{0}\right)$ or $\lambda(\mu, \xi ; a)$ for the principal Lyapunov exponent of (3.4) and (3.3).

Let

$$
\begin{array}{cc}
\hat{a}_{i}(x)=\lim _{t \rightarrow \infty} \frac{1}{t} \int_{0}^{t} a_{i}(s, x) d s, & \hat{a}_{0}(x)=\lim _{t \rightarrow \infty} \frac{1}{t} \int_{0}^{t} a_{0}(s, x) d s, \\
\check{a}_{i}(t)=\frac{1}{|D|} \int_{D} a_{i}(t, x) d x, & \check{a}_{0}(t)=\frac{1}{|D|} \int_{D} a_{0}(t, x) d x, \\
\bar{a}_{i}=\frac{1}{|D|} \int_{D} \hat{a}_{i}(x) d x, & \bar{a}_{0}=\frac{1}{|D|} \int_{D} \hat{a}_{0}(x) d x,
\end{array}
$$

where $i=1,2, \cdots, N$ and $|D|$ denotes the Lebesgue measure of $D$. Put

$$
\begin{aligned}
& \hat{c}_{l}(\xi):=c_{l}(\xi, \hat{a}):=\inf _{\mu>0} \frac{\lambda(\mu, \xi ; \hat{a})}{\mu}, \\
& \check{c}_{l}(\xi):=c_{l}(\xi ; \check{a}):=\inf _{\mu>0} \frac{\lambda(\mu, \xi ; \check{a})}{\mu},
\end{aligned}
$$

and

$$
\bar{c}_{l}(\xi):=c_{l}(\xi ; \bar{a}):=\inf _{\mu>0} \frac{\lambda(\mu, \xi ; \bar{a})}{\mu},
$$

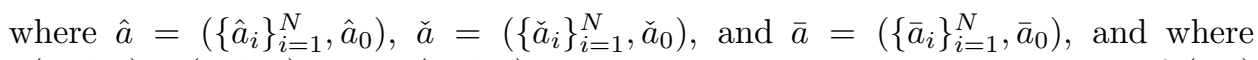
$\lambda(\mu, \xi ; \hat{a}), \lambda(\mu, \xi ; \check{a})$, and $\lambda(\mu, \xi ; \bar{a})$ are the principal Lyapunov exponents of (2.8) and (2.2), with $a$ being replaced by $\hat{a}, \check{a}$, and $\bar{a}$, respectively.

The main results of this section are stated in the following two theorems. 
Theorem 6.1 (Influence of time and space variation on linear spreading speed).

(1) If $a_{i}(t, x) \equiv a_{i}(t)(i=1,2, \cdots, N)$ and $a_{0}(t, x) \equiv a_{0}(t)$, then

$$
c_{l}(\xi)=\hat{c}_{l}(\xi) \quad \text { for any } \quad \xi \in S^{N-1} .
$$

(2) If $a_{i}(t, x) \equiv a_{i}(x)(i=1,2, \cdots, N)$, then

$$
c_{l}(\xi) \geq \hat{c}_{l}(\xi) \quad \text { for any } \quad \xi \in S^{N-1} .
$$

Moreover, $c_{l}(\xi)=\hat{c}_{l}(\xi)$ for some $\xi \in S^{N-1}$ if and only if $a_{0}(t, x)$ is of the form $a_{0}(t, x)=a_{01}(t)+a_{02}(x)$.

(3) If $a_{i}(t, x) \equiv a_{i}(i=1,2, \cdots, N)$ and $a_{0}(t, x) \equiv a_{0}(x)$, then

$$
c_{l}(\xi) \geq \check{c}_{l}(\xi) \quad \text { for any } \xi \in S^{N-1} \text {. }
$$

Moreover, $c_{l}(\xi)=\check{c}_{l}(\xi)$ for some $\xi \in S^{N-1}$ if and only if $a_{0}(x) \equiv a_{0}$.

(4) If $a_{i}(t, x) \equiv a_{i}(i=1,2, \cdots, N)$, then

$$
c_{l}(\xi) \geq \hat{c}_{l}(\xi) \geq \bar{c}_{l}(\xi) \quad \text { for any } \quad \xi \in S^{N-1} .
$$

Moreover, $c_{l}(\xi)=\hat{c}_{l}(\xi)=\bar{c}_{l}(\xi)$ for some $\xi \in S^{N-1}$ if and only if $a_{0}(t, x) \equiv$ $a_{0}(t)$.

Theorem 6.2 (Influence of time and space variation on spreading speeds).

(1) If $a_{i}(t, x) \equiv a_{i}(t)(i=1,2, \cdots, N)$ and $a_{0}(t, x) \equiv a_{0}(t)$, then

$$
c_{\mathrm{sup}}^{*}(\xi) \geq c_{\mathrm{inf}}^{*}(\xi) \geq \hat{c}_{l}(\xi) \quad \text { for any } \xi \in S^{N-1} .
$$

Moreover, if $f(t, x, u) \leq a_{0}(t) u$ for $t \in \mathbb{R}, x \in \mathbb{R}^{N}$, and $u \geq 0$, then

$$
c_{\mathrm{sup}}^{*}(\xi)=c_{\mathrm{inf}}^{*}(\xi)=\hat{c}_{l}(\xi) \quad \text { for any } \quad \xi \in S^{N-1} .
$$

(2) If $a_{i}(t, x) \equiv a_{i}(x)(i=1,2, \cdots, N)$ and $a_{0}(t, x)$ can be approximated by time periodic functions, then

$$
c_{\text {sup }}^{*}(\xi) \geq c_{\text {inf }}^{*}(\xi) \geq \hat{c}_{l}(\xi) \quad \text { for any } \quad \xi \in S^{N-1} .
$$

Moreover, if $f(t, x, u) \leq a_{0}(t, x) u$ for $t \in \mathbb{R}, x \in \mathbb{R}^{N}$, and $u \geq 0$, then

$$
c_{\mathrm{sup}}^{*}(\xi)=c_{\mathrm{inf}}^{*}(\xi) \geq \hat{c}_{l}(\xi) \quad \text { for any } \xi \in S^{N-1}
$$

and $\left(c_{\mathrm{sup}}^{*}(\xi)=\right) c_{\mathrm{inf}}^{*}(\xi)=\hat{c}_{l}(\xi)$ for some $\xi \in S^{N-1}$ if and only if $a_{0}(t, x)$ is of the form $a_{0}(t, x)=a_{01}(t)+a_{02}(x)$.

(3) If $a_{i}(t, x) \equiv a_{i}(i=1,2, \cdots, N)$ and $a_{0}(t, x) \equiv a_{0}(x)$, then

$$
c_{\mathrm{sup}}^{*}(\xi) \geq c_{\mathrm{inf}}^{*}(\xi) \geq \bar{c}_{l}(\xi) \quad \text { for any } \xi \in S^{N-1} .
$$

Moreover, if $f(t, x, u) \leq a_{0}(x) u$ for $t \in \mathbb{R}, x \in \mathbb{R}^{N}$, and $u \geq 0$, then

$$
c_{\mathrm{sup}}^{*}(\xi)=c_{\mathrm{inf}}^{*}(\xi) \geq \bar{c}_{l}(\xi) \quad \text { for any } \quad \xi \in S^{N-1}
$$

and $\left(c_{\mathrm{sup}}^{*}(\xi)=\right) c_{\mathrm{inf}}^{*}(\xi)=\bar{c}_{l}(\xi)$ for some $\xi \in S^{N-1}$ if and only if $a_{0}(x) \equiv a_{0}$ for $t \in \mathbb{R}$ and $x \in \mathbb{R}^{N}$.

(4) If $a_{i}(t, x) \equiv a_{i}(i=1,2, \cdots, N)$ and $a_{0}(t, x)$ can be approximated by time periodic functions, then

$$
c_{\mathrm{sup}}^{*}(\xi) \geq c_{\mathrm{inf}}^{*}(\xi) \geq \hat{c}_{l}(\xi) \geq \bar{c}_{l}(\xi) \quad \text { for any } \quad \xi \in S^{N-1} .
$$

Moreover, if $f(t, x, u) \leq a_{0}(t, x) u$ for $t \in \mathbb{R}, x \in \mathbb{R}^{N}$, and $u \geq 0$, then

$$
c_{\text {sup }}^{*}(\xi)=c_{\text {inf }}^{*}(\xi) \geq \hat{c}_{l}(\xi) \geq \bar{c}_{l}(\xi) \quad \text { for any } \quad \xi \in S^{N-1}
$$




$$
\begin{aligned}
& \text { and }\left(c_{\mathrm{sup}}^{*}(\xi)=\right) c_{\mathrm{inf}}^{*}(\xi)=\hat{c}_{l}(\xi)=\bar{c}_{l}(\xi) \text { for some } \xi \in S^{N-1} \text { if and only if } \\
& a_{0}(t, x) \equiv a_{0}(t) \text {. }
\end{aligned}
$$

Observe that

$$
\hat{a}_{0}(x)=\hat{f}_{u}(x, 0), \quad \check{a}_{0}(t)=\check{f}_{u}(t, 0), \quad \bar{a}_{0}=\bar{f}_{u}(0),
$$

where

$$
\begin{gathered}
\hat{f}(x, u)=\lim _{t \rightarrow \infty} \frac{1}{t} \int_{0}^{t} f(s, x, u) d s, \\
\check{f}(t, u)=\frac{1}{|D|} \int_{D} f(t, x, u) d x,
\end{gathered}
$$

and

$$
\bar{f}(u)=\frac{1}{|D|} \int_{D} \hat{f}(x, u) d x .
$$

If $f(t, x, u) \leq f_{u}(t, x, 0) u$ for $u \geq 0$ and $\hat{f}, \check{f}, \bar{f}$ are KPP type functions, then by Theorem 4.1 (3) and Theorem $5.1(3), \bar{c}_{l}(\xi), \check{c}_{l}(\xi)$ and $\bar{c}_{l}(\xi)$ are the spreading speeds of the following time-averaged, space-averaged, and time-space-averaged equations of (1.1),

$$
\begin{array}{cl}
\frac{\partial u}{\partial t}=\Delta u+\sum_{i=1}^{N} \hat{a}_{i}(x) \frac{\partial u}{\partial x_{i}}+\hat{f}(x, u), & x \in \mathbb{R}^{N}, \\
\frac{\partial u}{\partial t}=\Delta u+\sum_{i=1}^{N} \check{a}_{i}(t) \frac{\partial u}{\partial x_{i}}+\check{f}(t, u), & x \in \mathbb{R}^{N},
\end{array}
$$

and

$$
\frac{\partial u}{\partial t}=\Delta u+\sum_{i=1}^{N} \bar{a}_{i} \frac{\partial u}{\partial x_{i}}+\bar{f}(u), \quad x \in \mathbb{R}^{N},
$$

respectively. Therefore, Theorems 6.1 and 6.2 show that the time and space variation of the media cannot slow down the spatial spread; it indeed speeds up the spatial spread except in certain degenerate cases.

Proof of Theorem 6.1. (1) By Theorem 2.10 (1), for any $\mu \geq 0$ and $\xi \in S^{N-1}$,

$$
\lambda\left(\mu, \xi ; a_{0}\right)=\lim _{t-s \rightarrow \infty} \frac{1}{t-s} \int_{s}^{t}\left(a_{0}(\tau)-\mu \sum_{i=1}^{N} a_{i}(\tau) \xi_{i}+\mu^{2}\right) d \tau=\hat{a}_{0}-\mu \sum_{i=1}^{N} \hat{a}_{i} \xi_{i}+\mu^{2} .
$$

Then

$$
c_{l}(\xi)=\inf _{\mu>0} \frac{\hat{a}_{0}-\mu \sum_{i=1}^{N} \hat{a}_{i} \xi_{i}+\mu^{2}}{\mu}=\hat{c}_{l}(\xi)
$$

for any $\xi \in S^{N-1}$.

(2) By Theorem $2.10(2), \lambda(\mu, \xi ; a) \geq \lambda(\mu, \xi ; \hat{a})$ for any $\mu>0$ and $\xi \in \mathbb{R}^{N}$. Hence $c_{l}(\xi) \geq \hat{c}_{l}(\xi)$ for any $\xi \in S^{N-1}$. If $c_{l}(\xi)=\hat{c}_{l}(\xi)$ for some $\xi \in S^{N-1}$ and $a_{0}(t, x)$ is not of form $a_{0}(t, x)=a_{01}(t)+a_{02}(x)$, then by Theorem 2.10 (2) again, $\lambda(\mu, \xi ; a)>\lambda(\mu, \xi ; \hat{a})$ for any $\mu>0, \xi \in S^{N-1}$. Since $c_{l}(\xi)=\inf _{\mu>0} \frac{\lambda(\mu, \xi ; a)}{\mu}=$ $\inf _{\mu_{0}^{-} \leq \mu \leq \mu_{0}^{+}} \frac{\lambda(\mu, \xi ; a)}{\mu}$ (see Theorem 2.7), there is $\mu^{*} \in\left[\mu_{0}^{-}, \mu_{0}^{+}\right]$such that $c_{l}(\xi)=$ 
$\frac{\lambda\left(\mu^{*}, \xi ; a\right)}{\mu^{*}}$. But $\frac{\lambda\left(\mu^{*}, \xi ; a\right)}{\mu^{*}}>\frac{\hat{\lambda}\left(\mu^{*}, \xi ; \hat{a}\right)}{\mu^{*}}$. Then we must have $\hat{c}_{l}(\xi)<c_{l}(\xi)$, a contradiction. Therefore, if $c_{l}(\xi)=\hat{c}_{l}(\xi)$ for some $\xi \in S^{N-1}$, we must have $a_{0}(t, x)=$ $a_{01}(t)+a_{02}(x)$.

Conversely, if $a_{0}(t, x)=a_{01}(t)+a_{02}(x)$, then by Theorem 2.10 (2), $\lambda(\mu, \xi ; a)=$ $\lambda(\mu, \xi ; \hat{a})$ for any $\mu>0$ and $\xi \in S^{N-1}$. Hence $c_{l}(\xi)=\hat{c}_{l}(\xi)$ for any $\xi \in S^{N-1}$.

(3) By Theorem 2.10 (3), $\lambda(\mu, \xi ; a) \geq \lambda(\mu, \xi ; \check{a})$ for $\mu \geq 0$ and any $\xi \in S^{N-1}$ and $\lambda(\mu, \xi ; a)=\lambda(\mu, \xi ; \check{a})$ for some $\mu \geq 0$ and some $\xi \in S^{N-1}$ if and only if $a_{0}(x) \equiv a_{0}$. (3) then follows by the similar arguments as in (2).

(4) By $(2), c_{l}(\xi) \geq \hat{c}_{l}(\xi)$ for any $\xi \in S^{N-1}$. By $(3), \hat{c}_{l}(\xi) \geq \bar{c}_{l}(\xi)$ for any $\xi \in S^{N-1}$. It then follows that

$$
c_{l}(\xi) \geq \hat{c}_{l}(\xi) \geq \bar{c}_{l}(\xi)
$$

for any $\xi \in S^{N-1}$. Moreover, by $(2)$ and $(3), c_{l}(\xi)=\hat{c}_{l}(\xi)=\bar{c}_{l}(\xi)$ for some $\xi \in S^{N-1}$ if and only if $a_{0}(t, x)=a_{01}(t)+a_{02}(x)$ and $a_{02}(x) \equiv a_{02}$, hence if and only if $a_{0}(t, x) \equiv a_{0}(t)$.

Proof of Theorem 6.2. Observe that in any case, there holds

$$
c_{\text {sup }}^{*}(\xi) \geq c_{\text {inf }}^{*}(\xi) \text { for any } \xi \in S^{N-1} .
$$

(1) By Theorem 4.1 (2), we have

$$
c_{\mathrm{inf}}^{*}(\xi) \geq c_{l}(\xi)
$$

for any $\xi \in S^{N-1}$. By Theorem $6.1(1)$,

$$
c_{l}(\xi)=\hat{c}_{l}(\xi) \text { for } \quad \text { any } \quad \xi \in S^{N-1} .
$$

Hence

It then follows that

$$
c_{\mathrm{inf}}^{*}(\xi) \geq \hat{c}_{l}(\xi) \text { for any } \xi \in S^{N-1} \text {. }
$$

$$
c_{\mathrm{sup}}^{*}(\xi) \geq c_{\mathrm{inf}}^{*}(\xi) \geq \hat{c}_{l}(\xi) \text { for } \xi \in S^{N-1} .
$$

If $f(t, x, u) \leq a_{0}(t) u$ for $t \in \mathbb{R}, x \in \mathbb{R}^{N}$, and $u \geq 0$, then by Theorem 4.1 (3) and Theorem 6.1 (1),

$$
c_{\mathrm{sup}}^{*}(\xi)=c_{\mathrm{inf}}^{*}(\xi)=\hat{c}_{l}(\xi) \quad \text { for } \quad \text { any } \quad \xi \in S^{N-1} .
$$

(2) By Theorem $5.1(2)$,

$$
c_{\text {inf }}^{*}(\xi) \geq c_{l}(\xi) \text { for any } \xi \in S^{N-1} .
$$

By Theorem 6.1 (2),

$$
c_{l}(\xi) \geq \hat{c}_{l}(\xi) \text { for any } \xi \in S^{N-1} .
$$

It then follows that

$$
c_{\mathrm{sup}}^{*}(\xi) \geq c_{\mathrm{inf}}^{*}(\xi) \geq \hat{c}_{l}(\xi) \quad \text { for any } \quad \xi \in S^{N-1} .
$$

If in addition $f(t, x, u) \leq a_{0}(t, x) u$ for $t \in \mathbb{R}, x \in \mathbb{R}^{N}$, and $u \geq 0$, and $a_{0}(t, x)$ can be approximated by time periodic functions, then by Theorem 5.1 (3),

$$
c_{\mathrm{sup}}^{*}(\xi) \equiv c_{\mathrm{inf}}^{*}(\xi)=c_{l}(\xi) \quad \text { for any } \quad \xi \in S^{N-1} .
$$

This implies that $c_{\mathrm{inf}}^{*}(\xi)=\hat{c}_{l}(\xi)$ for some $\xi \in S^{N-1}$ if and only if $c_{l}(\xi)=\hat{c}_{l}(\xi)$ for some $\xi \in S^{N-1}$, which, by Theorem 6.1 (2) is equivalent to the fact that $a_{0}(t, x)$ is of the form $a_{0}(t, x)=a_{01}(t)+a_{02}(x)$. 
(3) It follows from Theorem 5.1 (2), (3), and Theorem 6.1 (3) and the similar arguments as in (2).

(4) It follows from Theorem 5.1 (2), (3), and Theorem 6.1 (4) and the similar arguments as in (2).

\section{Appendix A: Almost PeRiodic functions And COMPACT Flows}

In this section, we recall the definitions of compact flows and almost periodic functions and collect some basic properties.

Definition A.1. Let $Z$ be a compact metric space and $\mathcal{B}(Z)$ be the Borel $\sigma$-algebra of $Z$.

(1) $(Z, \mathbb{R}):=\left(Z,\left\{\sigma_{t}\right\}_{t \in \mathbb{R}}\right)$ is called a compact flow if $\sigma_{t}: Z \rightarrow Z(t \in \mathbb{R})$ satisfies: $\left[(t, z) \mapsto \sigma_{t} z\right]$ is jointly continuous in $(t, z) \in \mathbb{R} \times Z, \sigma_{0}=\mathrm{id}$, and $\sigma_{s} \circ \sigma_{t}=\sigma_{t+s}$ for any $s, t \in \mathbb{R}$. We may write $z \cdot t$ or $(z, t)$ for $\sigma_{t} z$.

(2) Assume that $\left(Z,\left\{\sigma_{t}\right\}_{t \in \mathbb{R}}\right)$ is a compact flow. A probability measure $\mathbb{P}$ on $(Z, \mathcal{B}(Z))$ is called an invariant measure for $\left(Z,\left\{\sigma_{t}\right\}_{t \in \mathbb{R}}\right)$ if for any $E \in \mathcal{B}(Z)$ and any $t \in \mathbb{R}, \mathbb{P}\left(\sigma_{t}(E)\right)=\mathbb{P}(E)$. An invariant measure $\mathbb{P}$ for $\left(Z,\left\{\sigma_{t}\right\}_{t \in \mathbb{R}}\right)$ is said to be ergodic if for any $E \in \mathcal{B}(Z)$ satisfying $\mathbb{P}\left(\sigma_{t}^{-1}(E) \triangle E\right)=0$ for all $t \in \mathbb{R}, \mathbb{P}(E)=1$ or $\mathbb{P}(E)=0$.

(3) Assume that $\left(Z,\left\{\sigma_{t}\right\}_{t \in \mathbb{R}}\right)$ is a compact flow. $\left(Z,\left\{\sigma_{t}\right\}_{t \in \mathbb{R}}\right)$ is said to be uniquely ergodic if it has a unique invariant measure (in such a case, the unique invariant measure is necessarily ergodic). We say that $\left(Z,\left\{\sigma_{t}\right\}_{t \in \mathbb{R}}\right)$ is minimal or recurrent if for any $z \in Z$, the orbit $\left\{\sigma_{t} z: t \in \mathbb{R}\right\}$ is dense in $Z$.

Given $g \in C\left(\mathbb{R} \times \mathbb{R}^{n}, \mathbb{R}^{m}\right)$, let

$$
H(g)=c l\{g \cdot \tau \mid \tau \in \mathbb{R}\},
$$

where $g \cdot \tau(t, x)=g(t+\tau, x)$ and the closure is taken under the compact open topology. $H(g)$ is usually called the hull of $g$. Let $\left(H(g),\left\{\sigma_{t}\right\}_{t \in \mathbb{R}}\right)$ be the translation flow defined by $\sigma_{t}(\tilde{g})=\tilde{g}(t+\cdot, \cdot)$ for $\tilde{g} \in H(g)$. If $g$ is bounded and uniformly continuous on $\mathbb{R} \times E$ for any bounded subset $E \subset \mathbb{R}^{n}$, then $H(g)$ is compact and metrizable under the compact open topology and hence $\left(H(g),\left\{\sigma_{t}\right\}_{t \in \mathbb{R}}\right)$ is a compact flow (see [54]).

Definition A.2. (1) A function $g \in C_{\text {unif }}\left(\mathbb{R}, \mathbb{R}^{m}\right)$ is said to be almost periodic if for any sequences $\left\{\alpha_{n}^{\prime}\right\},\left\{\beta_{n}^{\prime}\right\} \subset \mathbb{R}$, there are subsequences $\left\{\alpha_{n}\right\} \subset\left\{\alpha_{n}^{\prime}\right\}$, $\left\{\beta_{n}\right\} \subset\left\{\beta_{n}^{\prime}\right\}$ such that

$$
\lim _{k \rightarrow \infty} \lim _{n \rightarrow \infty} g\left(t+\alpha_{n}+\beta_{k}\right)=\lim _{n \rightarrow \infty} g\left(t+\alpha_{n}+\beta_{n}\right)
$$

pointwise for $t \in \mathbb{R}$.

(2) A function $g \in C\left(\mathbb{R} \times \mathbb{R}^{n}, \mathbb{R}^{m}\right),(t, x) \mapsto g(t, x)$, is said to be uniformly almost periodic in $t$ if $g$ is uniformly continuous on $\mathbb{R} \times E$ for any bounded subset $E \subset \mathbb{R}^{n}$ and is almost periodic in $t$ for each $x \in \mathbb{R}^{n}$.

(3) Let $g \in C\left(\mathbb{R} \times \mathbb{R}^{n}, \mathbb{R}^{m}\right)$ be uniformly almost periodic in $t$, and let

$$
g(t, x) \sim \sum_{\lambda \in \mathbb{R}} a_{\lambda}(x) e^{i \lambda t}
$$


be the Fourier series of $f$ (see [64, 73] for the definition). Then $\mathcal{S}(g)=$ $\left\{\lambda \mid a_{\lambda}(x) \not \equiv 0\right\}$ is called the Fourier spectrum of $g$, and $\mathcal{M}(g)=$ the smallest additive subgroup of $\mathbb{R}$ containing $\mathcal{S}(g)$ is called the frequency module of $g$.

Remark A.1. (1) If $g \in C_{\text {unif }}\left(\mathbb{R}, \mathbb{R}^{m}\right)$ is almost periodic, then for any sequences $\left\{\alpha_{n}^{\prime}\right\},\left\{\beta_{n}^{\prime}\right\} \subset \mathbb{R}$, there are subsequences $\left\{\alpha_{n}\right\} \subset\left\{\alpha_{n}^{\prime}\right\},\left\{\beta_{n}\right\} \subset\left\{\beta_{n}^{\prime}\right\}$ such that the limit in (A.1) is uniform in $t \in \mathbb{R}$ (see [13).

(2) Suppose that $g(t, x)\left(g \in C\left(\mathbb{R} \times \mathbb{R}^{n}, \mathbb{R}^{m}\right)\right)$ is uniformly almost periodic in $t$. Then $\left(H(g),\left\{\sigma_{t}\right\}_{t \in \mathbb{R}}\right)$ is minimal and uniquely ergodic (see [54, 73]).

(3) If $g(t, x)\left(g \in C\left(\mathbb{R} \times \mathbb{R}^{n}, \mathbb{R}^{m}\right)\right)$ is uniformly almost periodic in $t$, then $a_{\lambda}(x)$ in (A.2) is given by $a_{\lambda}(x)=\lim _{t \rightarrow \infty} \frac{1}{t} \int_{0}^{t} g(s, x) e^{-i \lambda s} d s$ (see [73]).

Definition A.3. A function $g \in C\left(\mathbb{R} \times \mathbb{R}^{n}, \mathbb{R}^{m}\right)$ is said to be recurrent in the first independent variable if it is bounded and uniformly continuous on $\mathbb{R} \times E$ for any bounded subset $E \subset \mathbb{R}^{n}$ and $\left(H(g),\left\{\sigma_{t}\right\}_{t \in \mathbb{R}}\right)$ is minimal.

Theorem A.1. If $g \in C\left(\mathbb{R} \times \mathbb{R}^{n}, \mathbb{R}\right)$ is uniformly almost periodic in $t$, then the limit $\lim _{t-s \rightarrow \infty} \frac{1}{t-s} \int_{s}^{t} g(\tau, x) d \tau$ exists for any $x \in \mathbb{R}$.

Proof. It follows from the results contained in [29].

\section{REFERENCES}

1. N. D. Alikakos, P. W. Bates, and X. Chen, Periodic traveling waves and locating oscillating patterns in multidimensional domains, Trans. Amer. Math. Soc., 351 (1999), 2777-2805. MR.1467460 (99j:35101)

2. D. G. Aronson and H. F. Weinberger, Nonlinear diffusion in population genetics, combustion, and nerve pulse propagation, in "Partial Differential Equations and Related Topics" (J. Goldstein, Ed.), Lecture Notes in Math., vol. 466, Springer-Verlag, New York, 1975, 5-49. MR.0427837 (55:867)

3. D. G. Aronson and H. F. Weinberger, Multidimensional nonlinear diffusions arising in population genetics, Adv. Math., 30 (1978), 33-76. MR511740(80a:35013)

4. H. Berestycki and F. Hamel, Generalized travelling waves for reaction-diffusion equations, Perspectives in nonlinear partial differential equations, 101-123, Contemp. Math., 446, Amer. Math. Soc., Providence, RI, 2007. MR2373726 (2009k:35135)

5. H. Berestycki, F. Hamel, and N. Nadirashili, The speed of propagation for KPP type problems. I. Periodic framework, J. Eur. Math. Soc., 7 (2005), 172-213. MR2127993 (2005k:35186)

6. H. Berestycki, F. Hamel, and N. Nadirashili, The speed of propagation for KPP type problems. II. General domains, preprint.

7. H. Berestycki, F. Hamel, and L. Roques, Analysis of periodically fragmented environment model. II. Biological invasions and pulsating traveling fronts, J. Math. Pures Appl., 84 (2005), 1101-1146. MR2155900 (2006d:35123)

8. X. Chen and J.-S. Guo, Existence and asymptotic stability of traveling waves of discrete quasilinear monostable equations, J. Diff. Eq., 184 (2002), no. 2, 549-569. MR1929888 (2003h:35107)

9. X. Chen and J.-S. Guo, Uniqueness and existence of traveling waves for discrete quasilinear monostable dynamics, Math. Ann., 326 (2003), no. 1, 123-146. MR1981615 (2004b:37175)

10. J. G. Conlon and C. R. Doering, On travelling waves for the stochastic Fisher-KolmogorovPetrovsky-Piscunov equation, J. Stat. Phys., 120 (2005), no. 3-4, 421-477. MR 2182316 (2006j:60064)

11. F. Dumortier, N. Popovic, and T. J. Kaper, The critical wave speed for the FisherKolmogorov-Petrowskii-Piscounov equation with cut-off, Nonlinearity, 20 (2007), no. 4, 855877. MR2307884

12. P. C. Fife and J. B. Mcleod, The approach of solutions of nonlinear diffusion equations to traveling front solutions, Arch. Ration. Mech. Anal., 65 (1977), 335-361. MR0442480 (56:862)

13. A. M. Fink, "Almost Periodic Differential Equations", Lecture Notes in Mathematics 377, Springer-Verlag, Berlin/Heidelberg/New York, 1974. MR0460799|(57:792) 
14. R. Fisher, The wave of advance of advantageous genes, Ann. of Eugenics, 7(1937), 335-369.

15. M. Freidlin and J. Gärtner, On the propagation of concentration waves in periodic and random media, Soviet Math. Dokl., 20 (1979), 1282-1286. MR553200(81d:80005)

16. A. Friedman, "Partial Differential Equations of Parabolic Type," Prentice-Hall, Inc., Englewood Cliffs, N.J., 1964. MR0181836 (31:6062)

17. J.-S. Guo and F. Hamel, Front propagation for discrete periodic monostable equations, Math. Ann., 335 (2006), no. 3, 489-525. MR2221123 (2007b:34071)

18. F. Hamel, Qualitative properties of monostable pulsating fronts: Exponential decay and monotonicity, prepint.

19. S. Heinze, G. Papanicolaou, and A. Stevens, A variational principle for propagation speeds in inhomogeneous media, SIAM J. Appl. Math., 62 (2001), 129-148. MR1857539 (2002j:35169)

20. D. Henry, "Geometric Theory of Semilinear Parabolic Equations", Lecture Notes in Math. vol. 840, Springer-Verlag, Berlin, 1981. MR610244 (83j:35084)

21. J. H. Huang and W. Shen, Speeds of spread and propagation for KPP models in time almost and space periodic media, preprint.

22. W. Hudson and B. Zinner, Existence of traveling waves for reaction diffusion equations of Fisher type in periodic media. Boundary value problems for functional-differential equations, World Sci. Publ., River Edge, NJ, 1995, 187-199. MR1375475 (97a:35112)

23. W. Hudson and B. Zinner, Existence of traveling waves for a generalized discrete Fisher's equation, Comm. Appl. Nonlinear Anal., 1 (1994), no. 3, 23-46. MR1295491 (95k:35110)

24. J. Húska, Harnack inequality and exponential separation for oblique derivative problems on Lipschitz domains, J. Diff. Eq., 226 (2006), no. 2, 541-557. MR.2237690 (2007h:35144)

25. J. Húska, Exponential separation and principal Floquet bundles for linear parabolic equations on general bounded domains: The divergence case, Indiana Univ. Math. J., 55 (2006), no. 3 , 1015-1043. MR 2244596 (2007d:35128)

26. J. Húska and P. Poláčik, The principal Floquet bundle and exponential separation for linear parabolic equations, J. Dynam. Diff. Eq., 16 (2004), no. 2, 347-375. MR2105779 (2006e:35147)

27. J. Húska, P. Poláčik and M. V. Safonov, Harnack inequality, exponential separation, and perturbations of principal Floquet bundles for linear parabolic equations, Ann. Inst. H. Poincaré Anal. Non Linéaire, 24 (2007), no. 5, 711-739. MR2348049 (2008k:35211)

28. V. Hutson, W. Shen, and G. Vickers, Estimates for the principal spectrum point for certain time-dependent parabolic operators, Proc. Amer. Math. Soc., 129 (2000), no. 6, 1669-1679. MR $1814096(2001 \mathrm{~m}: 35243)$

29. R. A. Johnson, K. J. Palmer and G. R. Sell, Ergodic properties of linear dynamical systems, SIAM J. Math. Anal., 18 (1987), no. 1, 1-33. MR871817(88a:58112)

30. Y. Kametaka, On the nonlinear diffusion equation of Kolmogorov-Petrovskii- Piskunov type, Osaka J. Math., 13 (1976), 11-66. MR0422875 (54:10861)

31. A. Kolmogorov, I. Petrowsky, and N. Piscunov, A study of the equation of diffusion with increase in the quantity of matter, and its application to a biological problem. Bjul. Moskovskogo Gos. Univ., 1 (1937), 1-26.

32. L. A. Lewis, B. Li, and H. F. Weinberger, Spreading speed and linear determinacy for two-species competition models, J. Math. Biol., 45 (2002), no. 3, 219-233. MR1930975 (2003h:92023)

33. B. Li, H. F. Weinberger, and L.A. Lewis, Spreading speeds as slowest wave speeds for cooperative systems, Math. Biosci., 196 (2005), no. 1, 82-98. MR2156610(2006g:92069)

34. X. Liang and X.-Q. Zhao, Asymptotic speeds of spread and traveling waves for monotone semiflows with applications, Comm. Pure Appl. Math., 60 (2007), no. 1, 1-40. MR2270161 (2007i:37144)

35. X. Liang, Y. Yi, and X.-Q. Zhao, Spreading speeds and traveling waves for periodic evolution systems, J. Diff. Eq., 231 (2006), no. 1, 57-77. MR2287877 (2008a:47110)

36. R. Lui, Biological growth and spread modeled by systems of recursions. Math. Biosciences, 93 (1989), 269-312. MR984281 (90g:92069)

37. S. Ma and X. Zou, Existence, uniqueness and stability of travelling waves in a discrete reactiondiffusion monostable equation with delay, J. Diff. Eq., 217 (2005), no. 1, 54-87. MR2170528 (2006g:35277)

38. A. J. Majda and P. E. Souganidis, Large scale front dynamics for turbulent reaction-diffusion equations with separated velocity scales, Nonlinearity, 7 (1994), 1-30. MR 1260130(95e:35180) 
39. A. J. Majda and P. E. Souganidis, Flame fronts in a turbulent combustion model with fractal velocity fields, Comm. Pure Appl. Math., 51 (1998), 13371348. MR1639220 (99f:35090)

40. H. Matano, Talks presented at various conferences.

41. J. Mierczyński, and W. Shen, Exponential separation and principal Lyapunov exponent/spectrum for random/nonautonomous parabolic equations, J.Diff. Eq., 191 (2003), 175205. MR 1973287 (2004h:35232)

42. J. Mierczyński and W. Shen, "Spectral Theory for Random and Nonautonomous Parabolic Equations and Applications," Chapman \& Hall/CRC Monogr. Surv. Pure Appl. Math., Chapman \& Hall/CRC, Boca Raton, FL, 2008.

43. C. Mueller and R. B. Sowers, Random travelling waves for the KPP equation with noise, $J$. Funct. Anal., 128 (1995), no. 2, 439-498. MR1319963 (97a:60083)

44. J. Nolen, M. Rudd, and J. Xin, Existence of KPP fronts in spatially-temporally periodic advection and variational principle for propagation speeds, Dynamics of PDE, 2 (2005), 1-24. MR 2142338 (2006h:35129)

45. J. Nolen and J. Xin, Existence of KPP type fronts in space-time periodic shear flows and a study of minimal speeds based on variational principle, Discrete Cont. Dynam. Syst., 13 (2005), 1217-1234. MR2166666 (2006e:35175)

46. J. Nolen and J. Xin, A variational principle based study of KPP minimal front speeds in random shears, Nonlinearity, 18 (2005), 1655-1675. MR2150348 (2006a:76058)

47. J. Nolen and J. Xin, A variational principle for KPP front speeds in temporally random shear flows, Comm. Math. Phys. 269 (2007), no. 2, 493-532. MR.2274555 (2007k:35237)

48. J. Nolen and J. Xin, Reaction-diffusion front speeds in spatially-temporally periodic shear flows, Multiscale Model. Simul. 1 (2003), 554-570. MR2029591 (2004j:35154)

49. P. Poláčik, On uniqueness of positive entire solutions and other properties of linear parabolic equations, Discrete Contin. Dyn. Syst., 12 (2005), no. 1, 13-26. MR 2121246 (2005k:35170)

50. P. Poláčik and I. Tereščák, Exponential separation and invariant bundles for maps in ordered Banach spaces with applications to parabolic equations, J. Dynam. Diff. Eq., 5 (1993), no. 2, 279-303; Erratum, J. Dynam. Diff. Eq., 6 (1994), no. 1, 245-246. MR1223450 (94d:47064)

51. N. Popovic and T. J. Kaper, Rigorous asymptotic expansions for critical wave speeds in a family of scalar reaction-diffusion equations, J. Dynam. Diff. Eq., 18 (2006), no. 1, 103-139. MR 2220193 (2006m:35196)

52. L. Ryzhik and A Zlatoš, KPP pulsating front speed-up by flows, Commun. Math. Sci., 5 (2007), no. 3, 575-593. MR2352332 (2008h:35200)

53. D. H. Sattinger, On the stability of waves of nonlinear parabolic systems, Advances in Math., 22 (1976), 312-355. MR0435602 (55:8561)

54. G. R. Sell, "Topological Dynamics and Ordinary Differential Equations", Van Nostrand Reinhold Company, 1971. MR0442908 (56:1283)

55 . W. Shen, Traveling waves in time almost periodic structures governed by bistable nonlinearities, I. Stability and Uniqueness, J. Diff. Eq., 159 (1999), 1-54. MR.1726918 (2001k:35157)

56 . W. Shen, Traveling waves in time almost periodic structures governed by bistable nonlinearities, II. Existence, J. Diff. Eq., 159 (1999), 55-101. MR.1726919 (2001k:35158)

57. W. Shen, Dynamical systems and traveling waves in almost periodic structures. J. Diff. Eq., 169 (2001), 493-548. MR1808473 (2003b:37124)

58. W. Shen, Traveling waves in diffusive random media, J. Dynam Diff. Eq., 16 (2004), 10111060. MR2110054 (2005k:35230)

59. W. Shen, Traveling waves in time dependent bistable equations, Diff. and Integral Eq., 19 (2006), 241-278. MR2215558 (2007a:35072)

60. W. Shen and Y. Yi, Almost automorphic and almost periodic dynamics in skew-product semiflows, Mem. Amer. Math. Soc. 136 (1998), no. 647. MR1445493 (99d:34088)

61. W. Shen and Y. Yi, Convergence in almost periodic Fisher and Kolmogorov models, J. Math. Biol. 37 (1998), 84-102. MR1636648 (99k:92037)

62. H. Thieme and X.-Q. Zhao, Asymptotic speeds of spread and traveling waves foor integral equations and delayed reaction-diffusion models, J. Diff. Eq., 195 (2003), 430-470. MR 2016819 (2004h:45007)

63. K. Uchiyama, The behavior of solutions of some nonlinear diffusion equations for large time, J. Math. Kyoto Univ., 183 (1978), 453-508. MR509494 (80g:35016)

64. W. A. Veech, Almost automorphic functions on groups, Amer. J. Math. 87 (1965), 719-751. MR0187014 (32:4469) 
65. H. F. Weinberger, Long-time behavior of a class of biology models, SIAM J. Math. Anal., 13 (1982), 353-396. MR653463 (83f:35019)

66. H. F. Weinberger, On spreading speeds and traveling waves for growth and migration models in a periodic habitat, J. Math. Biol., 45 (2002), 511-548. MR.1943224 (2004b:92043a)

67. H. F. Weinberger, M.A. Lewis, and B. Li, Analysis of linear determinacy for spread in cooperative models, J. Math. Biol., 45 (2002), no. 3, 183-218. MR.1930974 (2003h:92029)

68. J. Wu and X. Zou, Asymptotic and periodic boundary value problems of mixed FDEs and wave solutions of lattice differential equations, J. Diff. Eq., 135 (1997), no. 2, 315-357. MR.1441274 (98f:34099)

69. J. Wu and X. Zou, Traveling wave fronts of reaction-diffusion systems with delays, J. Dynam. Diff. Eq., 13 (2001), 651-687. MR 1845097 (2003a:35114)

70. J. Xin, Existence and stability of traveling waves in periodic media governed by a bistable nonlinearity, J. Dynamics Diff. Eq. 3 (1991) no. 4, 541-573. MR1129560 (92k:35150)

71. J. Xin, Front propagation in heterogeneous media, SIAM Review, 42 (2000), 161-230. MR.1778352 (2001i:35184)

72. J. Xin, KPP front speeds in random shears and the parabolic Anderson problem, Methods and Applications of Analysis, 10 (2003), 191-198. MR2074747 (2005g:35182)

73. Y. Yi, Almost automorphic and almost periodic dynamics in skew-product semiflows, Part I. Almost automorphy and almost periodicity, Mem. Amer. Math. Soc. 136 no. 647 (1998). MR.1445493 (99d:34088)

74. B. Zinner, G. Harris, and W. Hudson, Traveling wavefronts for the discrete Fisher's equation, J. Diff. Eq., 105 (1993), no. 1, 46-62. MR.1237977(94k:39034)

75. A. Zlatoš, Pulsating front speed-up and quenching of reaction by fast advection, Nonlinearity 20 (2007), 2907-2921. MR2368331 (2008j:35105)

Department of Mathematics and Statistics, Auburn University, Auburn, Alabama 36849

E-mail address: wenxish@auburn.edu 